\title{
TABLE OF RADIOISOTOPES ARRANGED ACCORDING TO HALF-LIFE
}

\author{
Compiled \\ by
}

Elaine Daniels

From the General Electric Chart of the Nuclides

Fifth Edition (April, 1956)

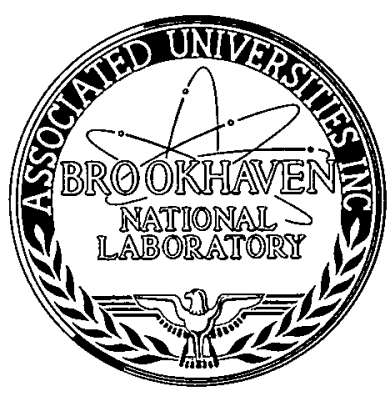

December, 1957
BROOKHAVEN NATIONAL LABORATORY
Associated Universities, Inc. under contract with the
United States Atomic Energy Commission




\section{DISCLAIMER}

This report was prepared as an account of work sponsored by an agency of the United States Government. Neither the United States Government nor any agency Thereof, nor any of their employees, makes any warranty, express or implied, or assumes any legal liability or responsibility for the accuracy, completeness, or usefulness of any information, apparatus, product, or process disclosed, or represents that its use would not infringe privately owned rights. Reference herein to any specific commercial product, process, or service by trade name, trademark, manufacturer, or otherwise does not necessarily constitute or imply its endorsement, recommendation, or favoring by the United States Government or any agency thereof. The views and opinions of authors expressed herein do not necessarily state or reflect those of the United States Government or any agency thereof. 


\section{DISCLAIMER}

Portions of this document may be illegible in electronic image products. Images are produced from the best available original document. 


\title{
TABLE OF RADIOISOTOPES \\ ARRANGED ACCORDING TO HALF-LIFE
}

\author{
Compiled \\ by \\ Elaine Daniels
}

From the General Electric Chart of the Nuclides

Fifth Edition (April, 1956)

December, 1957

BROOKHAVEN NATIONAL LABORATORY

Upton, N.Y. 


\section{LEGAL NOTICE}

This report was prepared as an account of Government sponsored work. Neither the United States, nor the Commission, nor any person acting on behalf of the Commission:

A. Makes any warranty or representation, express or implied, with respect to the accuracy, completeness, or usefulness of the information contained in this report, or that the use of any information, apparatus, method, or process disclosed in this report may not infringe privately owned rights; or

B. Assumes any liabilities with respect to the use of, or for damages resulting from the use of any information, apparatus, method, or process disclosed in this report.

As used in the above "person acting on behalf of the Commission" includes any employee or contractor of the Commission to the extent that such employee or contractor prepares, handles or distributes, or provides access to, any information pursuant to his employment or contract with the Commission.

PRICE $\$ 1.75$

Available from the

Office of Technical Services,

Department of Commerce,

Washington 25, D.C.

February 1958

850 copies 
The data in this table were taken directly from the General Electric Chart of the Muclides, Fifth Edition, (revised to April, 1956). This Chart of the Nuclides was originally prepared by G. Friedlander and M. Perlman (Brookhaven National Laborationy) and subsequently revised by J. R. Stehn and E. F. Clancy (Knolls Atomic Power Laboratory).

Great care has been used in copying the data and in proof-reading the copy to eliminate errors in transcription. On the other hand no respons 1bility can be assumed for any errors which may exist in the chart 1tself.

The symbols and abbreviations used herein are the same as used in the Chart of the Nuclides:

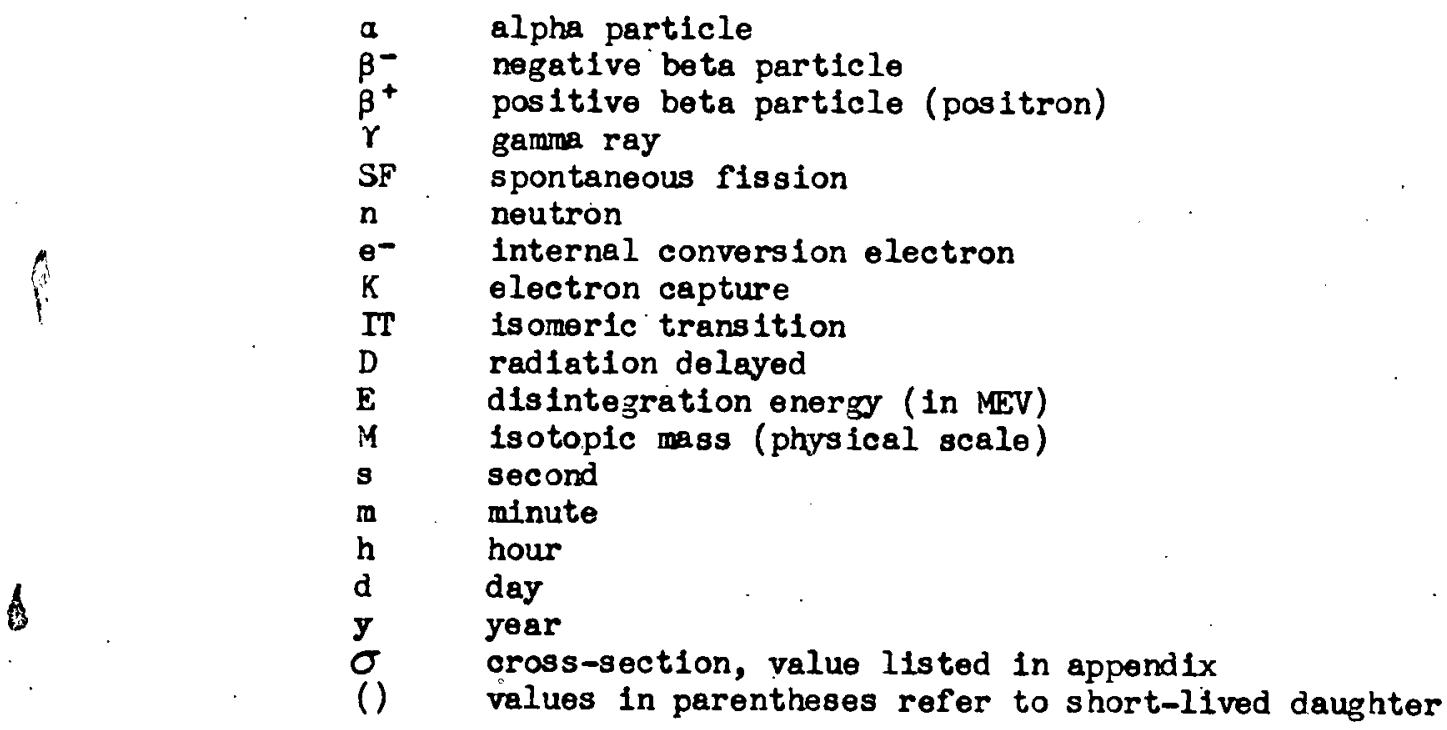

Frequently a motastable state is denoted by a letter m following the mass number. This convention was not used in the present compilation. However, this should create no confusion since a glance at column 4 will show whether the isotope decays by isomeric transition and, therefore, whether it is in a metastable sta.te.

Where the Chart of the Nuclides lists a cross-section value for the radiolsotope a symbol $\sigma$ appears just to the left of column 4. The value for this cross-section may be found in the Appendix where the isotope is listed by atomic number.

The advice and help of L. G. Stang, Jr. and W. D. Tucker is acknowledged.

This work was carried out under the auspices of the Atomic Energy Commission. 


\begin{tabular}{|c|c|c|c|c|c|}
\hline Half-Ltfe & Isotope & $\begin{array}{l}\text { Particles and } \\
\text { Energies (Mev) }\end{array}$ & & $\begin{array}{l}\text { Camma Energies } \\
\frac{(\mathrm{Mev})}{}\end{array}$ & $\begin{array}{l}\text { Total Enern } \\
\text { or Mass }\end{array}$ \\
\hline $2 \times 10^{-21} \mathrm{~s}$ & 5 & $\mathrm{n}, \boldsymbol{a}$ & & . & M 5.0139 \\
\hline$\sim 10^{-21} \mathrm{~s}$ & $\mathrm{LH}^{5}$ & $p, a$ & & & M 5.0139 \\
\hline$\geq 3 \times 10^{-19} \mathrm{~s}$ & $\mathrm{~B}^{9}$ & $\stackrel{p}{(2} a)$ & & & $M 9.0162$ \\
\hline$<4 \times 10^{-15} \mathrm{~s}$ & $B \theta^{8}$ & $2 \propto .09$ & & & M 8.00785 \\
\hline $3 \times 10^{-7} \mathrm{~s}$ & $\mathrm{Po}^{212}$ & a 8.78 & & . & M 212.055 \\
\hline $4 \times 10^{-6} \mathrm{~s}$ & $\mathrm{Po}^{213}$ & a 8.34 & & & M 213.059 \\
\hline $1.6 \times 10^{-4} \mathrm{~s}$ & $\mathrm{Po}^{214}$ & a 7.68 & & & M 214.062 \\
\hline$\sim 10^{-4} \mathrm{~s}$ & $A t^{215}$ & a 8.00 & & & M 215.066 \\
\hline$\sim 3 \times 10^{-4} \mathrm{~s}$ & $A t^{216}$ & a 7.79 & & & M 216.070 \\
\hline$\sim 10^{-3} \mathrm{~s}$ & $\mathrm{Em}^{217}$ & a 7.74 & & & M 217.072 \\
\hline $1.8 \times 10^{-3} \mathrm{~s}$ & $\mathrm{Po}^{215}$ & $\beta^{-7.36}$ & & & M 215.067 \\
\hline $5 \times 10^{-3} \mathrm{~s}$ & $w^{180}$ & & & $\begin{array}{l}\text { I.T. } \\
\mathrm{r} .35, .22\end{array}$ & \\
\hline $1.2 \times 10^{-2} \mathrm{~s}$ & $\mathrm{~N}^{12}$ & $\begin{array}{l}\beta^{+} 16.7 \\
(3 a \sim 4)\end{array}$ & & & E 17.7 \\
\hline $1.8 \times 10^{-2} \mathrm{~s}$ & $\mathrm{As}^{75}$ & . & & I.T. .28 & \\
\hline $1.8 \times 10^{-2} \mathrm{~s}$ & $A t^{217}$ & $a 7.0 R$ & & & M 217.073 \\
\hline $1.9 \times 10^{-2} \mathrm{~s}$ & $\mathrm{Em}^{218}$ & $a 7.13,6.53$ & · & $r .61$ & M 218.074 \\
\hline $2 \times 10^{-2} \mathrm{~s}$ & $\mathrm{Fr}^{219}$ & a 7.30 & & & M 219.078 \\
\hline $2.5 \times 10^{-2} \mathrm{~s}$ & $\mathrm{~B}^{12}$ & $\beta-13.4,9.0$ & & $r 4.4$ & E 13.4 \\
\hline $0.16 \mathrm{~s}$ & $\mathrm{Po}^{216}$ & $\alpha 6.77$ & & & M 216.069 \\
\hline $0.17 \mathrm{~s}$ & $\mathrm{u}^{9}$ & $\begin{array}{l}\beta^{-} \\
(n, 2 a)\end{array}$ & ; & & E 14 \\
\hline $0.18 \mathrm{~s}$ & $\mathrm{Co}^{54}$ & $\beta^{+}>7$ & . & & \\
\hline $0.2 \mathrm{~s}$ & $\mathrm{Sc}^{40}$ & $\beta^{+} 9$ & & $r 3.7$ & E $\mathbf{U}_{4}$ \\
\hline
\end{tabular}

- Total Disintegration Energy (Mev), or Isotopic Mass 


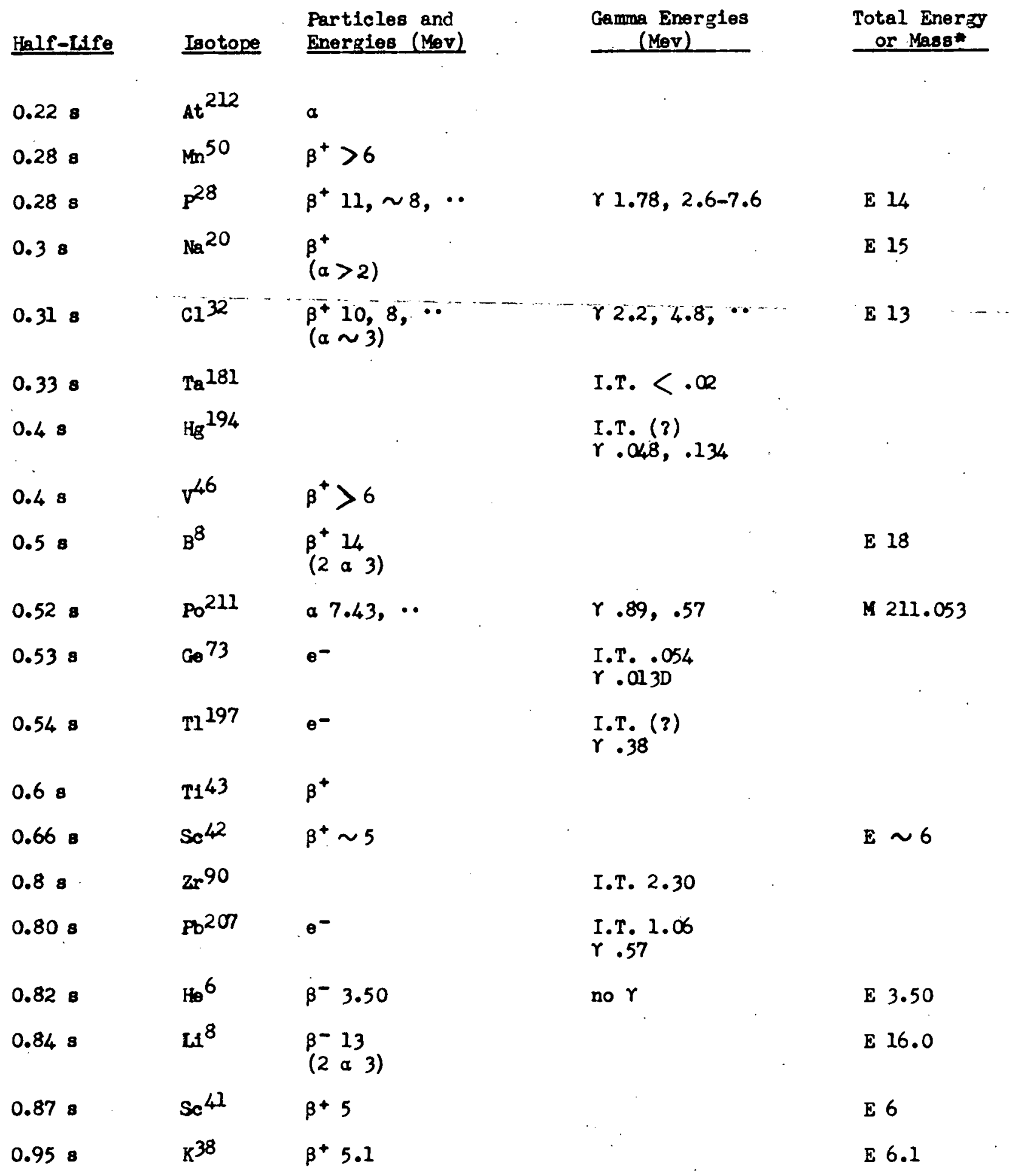

* Total Disintegration Energy (Mov), or Isotopic Mass 


\begin{tabular}{|c|c|c|c|c|}
\hline Half-Lifo & Isotope & $\begin{array}{l}\text { Particles and } \\
\text { Energies (Mav) }\end{array}$ & $\begin{array}{c}\text { Canme Energies } \\
(\mathrm{Mov})\end{array}$ & $\begin{array}{l}\text { Total Energy } \\
\text { or Mags } \\
\end{array}$ \\
\hline 18 & $\mathrm{Cl} 38$ & & I.T. .66 & \\
\hline $1 \mathrm{~s}$ & $x_{\theta}{ }^{1 / 3}$ & $\beta^{-}$ & & \\
\hline 18 & $K r^{94}$ & $\beta^{-}$ & & \\
\hline$\sim 18$ & $k r^{97}$ & $\beta^{-}$ & & \\
\hline$\sim 1 s$ & $x_{\theta}{ }^{1 / 4}$ & $\beta^{-}$ & & \\
\hline$\sim 1 \mathrm{~s}$. & $v^{45}$ & $\beta^{+}$ & & \\
\hline $1.0 \mathrm{~s}$ & $\mathrm{Ca}^{39}$ & $\beta^{-} 5.7$ & & E 6.7 \\
\hline $1.1 \mathrm{~s}$ & $C r^{46}$ & $\beta^{+}$ & & \\
\hline 1.38 & $A t^{218}$ & $a 6.63$ & $\cdot$ & M 218.077 \\
\hline $1.5 \mathrm{~s}$ & $\mathrm{Cl}^{34}$ & $\beta^{+} 4.5$ & & E 5.5 \\
\hline $1.6 \mathrm{~s}$ & $\mathrm{Ne}^{18}$ & $\beta^{+} 3.2$ & & E 4.2 \\
\hline $1.7 \mathrm{~s}$ & $S 1^{26}$ & $\beta^{+}$ & & \\
\hline $1.8 \mathrm{~s}$ & $A^{35}$ & $\beta+4.95$ & $r 1.22,1.76$ & E 5.97 \\
\hline$<2 \mathrm{~s}$ & $A c^{221}$ & a 7.6 & & $M 221.085$ \\
\hline$<28$ & $\operatorname{Fr}^{217}$ & a 8.3 & & M 217.073 \\
\hline$<2 \mathrm{~s}$ & $\dot{A} t^{213}$ & a 9.2 & & $M 213.060$ \\
\hline 28 & $\mathrm{Kr} 93$ & $\beta^{-}$ & $\because$ & \\
\hline $2.0 \mathrm{~s}$ & $\mathrm{~Pa}^{225}$ & $\alpha$ & & \\
\hline $2.1 \mathrm{~s}$ & $\mathrm{Al}^{24}$ & $\begin{array}{l}\beta^{+}<8.5,8.5 \\
(\alpha \sim 2)\end{array}$ & $\gamma 1.38-7.2$ & E 14 \\
\hline $2.3 \mathrm{~s}$ & $c^{15}$ & $\beta^{-} 4.3,9.8$ & $r 5.3$ & E 9.8 \\
\hline $2.5 \mathrm{~s}$ & $\operatorname{In}^{112}$ & $e^{-}$ & I.T. . .15 & \\
\hline $2.5 \mathrm{~s}$ & $\mathrm{Er}^{2}$ & $\theta^{-}$ & I.T. . 21 & . \\
\hline $\begin{array}{c}2.6 \mathrm{~s} \\
6\end{array}$ & $s^{31}$ & $\beta^{+} 4.4$ & & E 5.4 \\
\hline
\end{tabular}




\begin{tabular}{|c|c|c|c|c|}
\hline Half-Life & Isotope & $\begin{array}{l}\text { Particles and } \\
\text { Energies (Mov) }\end{array}$ & $\begin{array}{l}\text { Gamma Energies } \\
\text { (Mev) }\end{array}$ & $\begin{array}{l}\text { Total Enersy } \\
\text { or Mess* }\end{array}$ \\
\hline $2.7 \mathrm{~s}$ & $I^{139}$ & $\beta^{-}$ & & \\
\hline $2.8 \mathrm{~s}$ & $\mathrm{Cl}^{33}$ & $\beta^{+} 4.2$ & $\gamma 2.8 ?$ & E 5.2 \\
\hline $3 s$ & $\mathrm{Cu}^{58}$ & $\beta^{+} \sim 8$ & & \\
\hline $3 \mathrm{~s}$ & $\mathrm{xe}^{1 / 41}$ & $\beta^{-}$ & & \\
\hline $3 \mathrm{~s}$ & $\mathrm{Kr} 92$ & $\beta^{-}$ & $\begin{array}{lll}\cdots & \cdots & -\end{array}$ & $\ldots \ldots$ \\
\hline $3.92 \mathrm{~s}$ & $\operatorname{En}^{219}$ & a $6.82,6.56, \cdots$ & $\gamma .27, .40, \cdots$ & M 219.078 \\
\hline $4 \mathrm{~s}$ & $\mathrm{Au}^{193}$ & $e^{-}$ & $\underset{r . T .}{\text { I.T. }} .032, .290$ & \\
\hline $4.14 \mathrm{~s}$ & $\mathrm{~N}^{17}$ & $\begin{array}{l}8^{-}-3.7 \\
(\mathrm{n} 1.0)\end{array}$ & & E 8.8 \\
\hline 4.48 & $S i^{27}$ & $\beta^{+} 3.8$ & & E 4.8 \\
\hline $4.5 \mathrm{~s}$ & $P^{29}$ & $\beta^{+} 3.94, \cdots$ & $\gamma 1.28,2.43$ & E 4.96 \\
\hline $4.5 \mathrm{~s}$ & $\mathrm{Br}^{89}$ & $\begin{array}{l}\beta^{-} \\
(n \cdot 5)\end{array}$ & & \\
\hline$<5 \mathrm{~s}$ & $\mathrm{Fr}^{218}$ & a 7.85 & 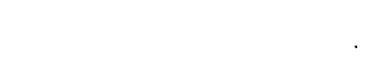 & M 218.076 \\
\hline$<5 \mathrm{~s}$ & $A t^{214}$ & $\alpha 8.78$ & & $M 214.063$ \\
\hline $5 \mathrm{~s}$ & $F^{21}$ & $\beta^{-}$ & & E 5.7 \\
\hline $5 \mathrm{~s}$ & $\operatorname{Ir}{ }^{191}$ & $e^{-}$ & ${ }_{Y . T .129}^{\text {I.T. }}$ & \\
\hline $5 \mathrm{~s}$ & $\mathrm{Te}^{10 R}$ & $\beta^{-} 4$ & & 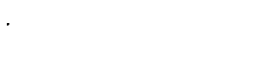 \\
\hline $5.5 \mathrm{~s}$ & $w^{183}$ & $e^{-}$ & $\begin{array}{l}\text { I.T. } \\
r .11, .16\end{array}$ & \\
\hline $5.5 \mathrm{~s}$ & $\mathrm{Ac}^{222}$ & a 6.96 & & M 222.087 \\
\hline $5.9 \mathrm{~s}$ & $\mathrm{I}^{138}$ & $\beta^{-}$ & & \\
\hline $6 s$ & $\mathrm{~Pb}^{203}$ & $e^{-}$ & I.T. . 86 & \\
\hline $6 s$ & $\mathrm{Yb}^{3}$ & & $\underset{\gamma .10 ?}{\text { I.T. }}$ & \\
\hline
\end{tabular}

- Total Disintegration Energy (Mev), or Isotopic Mass 


\begin{tabular}{|c|c|c|c|c|}
\hline Helf-Life & Isotope & $\begin{array}{l}\text { Particles and } \\
\text { Energies (Mov) }\end{array}$ & $\begin{array}{l}\text { Gamma Energies } \\
\frac{\text { (Mev) }}{}\end{array}$ & $\begin{array}{l}\text { Total En } \\
\text { or Mas }\end{array}$ \\
\hline $6.5 \mathrm{~s}$ & $\mathrm{Al}^{26}$ & $\beta^{+} 3.21$ & & E 4.23 \\
\hline $7.3 \mathrm{~s}$ & $\mathrm{Al}^{25}$ & $\beta^{+} 3.24$ & & E 4.26 \\
\hline $7.4 \mathrm{~s}$ & $\mathrm{Au}^{197}$ & & $\underset{\gamma .279}{\text { I.T. } .130, .409}$ & \\
\hline $7.4 \mathrm{~s}$ & $\mathrm{~N}^{16}$ & $\beta^{-} 4,10.4, \cdots$ & $\gamma 6.13,7.10,2.7, \ldots$ & E 10.4 \\
\hline $10 \mathrm{~s}$ & $\mathrm{Kr}^{91}$ & $\beta-3.6$ & $r$ & \\
\hline $11 \mathrm{~s}$ & $\mathrm{~F}^{20}$ & $\beta^{-} 5.42$ & $r 1.63$ & E 7.05 \\
\hline $12 \mathrm{~s}$ & $\mathrm{Mg}^{23}$ & $\beta^{+} 3.0$ & & $E 4.0$ \\
\hline $12.4 \mathrm{~s}$ & $\mathrm{P} 34$ & $\beta^{-} 5.1,3.2$ & $r 2.1,4.0$ & E 7.2 \\
\hline $13 \mathrm{~s}$ & $\mathrm{Kr}^{81}$ & & I.T. .19 & \\
\hline $13 \mathrm{~s}$ & $\operatorname{In}^{116}$ & $\beta^{-} 3.3$ & no $\gamma$ & E 3.3 \\
\hline $16 \mathrm{~s}$ & $\mathrm{Y}^{89}$ & & I.T. .91 & \\
\hline $16 s$ & $\mathrm{Br}^{88}$ & $\beta^{-}$ & & \\
\hline $16 \mathrm{~s}$ & $\mathrm{Xe}^{140}$ & $\beta^{-}$ & & \\
\hline $16 \mathrm{~s}$ & $\operatorname{Te}^{100}$ & $\beta^{-} 2.8$ & $\gamma .54$ & \\
\hline $17 \mathrm{~s}$ & $S e^{77}$ & & I.T. . .16 & \\
\hline $18 \mathrm{~s}$ & $\mathrm{Rh}^{108}$ & $\beta^{-} \sim 4$ & $\gamma$ & \\
\hline $18.5 \mathrm{~s}$ & $\mathrm{Ne}^{19}$ & $\beta+2.2$ & & E 3.2 \\
\hline $19 \mathrm{~s}$ & $\mathrm{Hr}^{179}$ & $e^{-}$ & $\underset{Y}{\mathrm{I} . \mathrm{T} .22} \cdot .16$ & \\
\hline $19 \mathrm{~s}$ & $c^{10}$ & $\beta^{+} 1.9, \cdots$ & $r .72,1.03$ & E 3.6 \\
\hline $20 \mathrm{~s}$ & $\mathrm{Se}^{46}$ & $e^{-}$ & I.T. .14 & \\
\hline $22 \mathrm{~s}$ & $\mathrm{I}^{137}$ & $\begin{array}{l}\beta^{-} \\
(n \cdot .6)\end{array}$ & & \\
\hline $23 \mathrm{~s}$ & $\mathrm{Pd}^{105}$ & $e^{-}$ & I.T. .2 & \\
\hline
\end{tabular}

* Total Disintegration Energy (Kev), or Isotopic Mass

Page 5. 


\begin{tabular}{|c|c|c|c|c|}
\hline Half-Iffe & Isotope & $\begin{array}{l}\text { Particles and } \\
\text { Energies (Mev) }\end{array}$ & $\begin{array}{l}\text { Gamme Energies } \\
\frac{(\mathrm{MaV})}{}\end{array}$ & $\begin{array}{l}\text { Total Ener 5y } \\
\text { or Mass* }\end{array}$ \\
\hline $23 \mathrm{~s}$ & $\mathrm{Na}^{21}$ & $\beta^{+} 2.50$ & & E 3.52 \\
\hline 243 & $\mathrm{Nb}^{90}$ & $e^{-}$ & I.T. .12 & \\
\hline 248 & $A B^{110}$ & $\beta^{-} 2.22,2.88, \cdots$ & $r .66, \cdots$ & E 2.88 \\
\hline$\sim 258$ & $\mathrm{Au}^{2 \mathscr{R}}$ & $\beta^{-}$ & & \\
\hline $25 \mathrm{~s}$ & $\mathrm{Po}^{211}$ & a $7.1,8.7, \ldots$ & $(\gamma 1.06, .57)$ & $\because \quad-\cdots$ \\
\hline $28 \mathrm{~s}$ & $\mathrm{Fr}^{220}$ & a 6.69 & & M 220.081 \\
\hline $29 \mathrm{~s}$ & $0^{19}$ & $\beta^{-}-3.2,4.4$ & $r .1970,1.37, .110$ & E 4.79 \\
\hline$<30 \mathrm{~s}$ & $\mathrm{Ba}^{143}$ & $\beta^{-}$ & & \\
\hline $30 \mathrm{~s}$ & $\mathrm{Ra}^{221}$ & a 6.71 & & M 221.083 \\
\hline $30 \mathrm{~s}$ & $\mathrm{Au}^{195}$ & $e^{-}$ & ${ }_{r . T .}^{\text {I.T. }} .057, .318$ & \\
\hline $30 \mathrm{~s}$ & $\mathrm{Rh}^{105}$ & $e^{-}$ & I.T. .130 & \\
\hline $30 \mathrm{~s}$ & $\mathrm{Rh}^{106}$ & $\beta^{-} 3.53, \cdots$ & $Y .51, .62, .87-2.66$ & E 3.53 \\
\hline $32 \mathrm{~s}$ & $\mathrm{Cu}^{68}$ & $\beta^{-} 3.0$ & $\gamma$ & \\
\hline 338 & $\mathrm{Kr}^{90}$ & $\beta^{-} 3.2$ & $\gamma$ & \\
\hline$\sim 368$ & $\mathrm{As}^{80}$ & $\beta^{-}$ & & \\
\hline $38 \mathrm{~s}$ & $\mathrm{Ra}^{222}$ & a $6.55,6.23$ & $r .33$ & M 222.085 \\
\hline 408 & $\mathrm{Ne}^{23}$ & $\beta^{-} 4.2,3.8, \cdots$ & $r .44,1.65, \cdots$ & E 4.2 \\
\hline $40 \mathrm{~s}$ & $A g 109$ & $\theta^{-}$ & I.T. .088 & \\
\hline 428 & $\mathrm{Hg}<195$ & a 5.6 & & \\
\hline 428 & $\operatorname{Rh}^{104}$ & $\beta-2.5, \cdots$ & $r .55,1.24$ & E 2.5 \\
\hline 438 & $\Delta_{t}<2 \mathbb{R}$ & a 6.50 & & \\
\hline 448 & $A B^{107}$ & $e^{-}$ & I.T. .093 & \\
\hline $48 \mathrm{~s}$ & $\mathrm{Sb}^{13 / 4}$ & $\beta^{-}$ & & \\
\hline 498 & $\mathrm{Ce}^{75}$ & $e^{-}$ & I.T. .14 & \\
\hline
\end{tabular}

Page 6. 


\begin{tabular}{|c|c|c|c|c|}
\hline Llf-Life & Isotope & $\begin{array}{l}\text { Particles and } \\
\text { Energies (Mov) }\end{array}$ & $\begin{array}{l}\text { Gamma Energies } \\
\text { (Mev) }\end{array}$ & $\begin{array}{l}\text { Total Energy } \\
\text { or Mass* }\end{array}$ \\
\hline $50 \mathrm{~s}$. & $I r^{198}$ & $\beta-3.6$ & $\gamma .78$ & E 4.4 \\
\hline $50 \mathrm{~s}$ & $\mathrm{Yb}^{3}$ & $e^{-}$ & I.T. .®5 & \\
\hline $52 \mathrm{~s}$ & $\sin ^{220}$ & $\alpha 6.28,5.75$ & & M 220.080 \\
\hline $52 \mathrm{~s}$ & $\mathrm{Ce}^{77}$ & $\beta-2.9, \cdots$ & $\begin{array}{l}\text { I.T. } \\
r .21\end{array}$ & \\
\hline $54 \mathrm{~s}$ & $\operatorname{Par}<94$ & $\beta^{+}$ & $r$ & \\
\hline $54 \mathrm{~s}$ & $A t^{219}$ & $\beta^{-6.27}$ & & M 219.080 \\
\hline $55 \mathrm{~s}$ & $\mathrm{Au}^{203}$ & $\beta^{-} 1.9$ & $r .69$ & E 1.9 \\
\hline 558 & $x_{e}^{125}$ & & ${ }_{r}^{\text {I.T.i. }} .075$ & . \\
\hline $55 \mathrm{~s}$ & $v^{54}$ & $\beta^{-3} 3.3$ & $r .99, .84,2.21$ & \\
\hline $55 \mathrm{~s}$ & $\mathrm{Kr}^{79}$ & & I.T. . .13 & \\
\hline $56 \mathrm{~s}$ & $\mathrm{Br} 87$ & $\begin{array}{l}\beta^{-2.6}, 8.0 \\
(n \cdot 3)\end{array}$ & $r 3.2,5.4$ & E 8.0 \\
\hline$<1 \mathrm{~m}$ & $\operatorname{Em}^{215}$ & a 8.6 & . & M 215.066 \\
\hline$<1 \mathrm{~m}$ & $\mathrm{Th}^{223}$ & a 7.5 & & M 223.091 \\
\hline$<1$ m & $\mathrm{Re}^{219}$ & a 8.0 & & M 219.079 \\
\hline$<1$ m & $A c^{230}$ & $\beta^{-} 2.2$ & & \\
\hline$<1$ m & In 118 & $\beta^{-4}$ & & \\
\hline$\sim 1 \mathrm{~m}$ & $\operatorname{Cs} 142$ & $\beta^{-}$ & & \\
\hline 1 m & $\mathrm{Na} 25$ & $\beta^{-} 4.0,2.6-3.4$ & $r .98, .58, .38,1.60$ & E 4.0 \\
\hline $1 \mathrm{~m}$ & $\mathrm{NB}^{97}$ & $e^{-}$ & I.T. .75 & \\
\hline $2.0 \mathrm{~m}$ & $\mathrm{FB}^{201}$ & $\theta^{-}$ & I.T. .66 & \\
\hline $1.0 \mathrm{~m}$ & $R b^{86}$ & & I.T. . .56 & \\
\hline $1.1 \mathrm{~m}$ & $\mathrm{P}^{17}$ & $\beta^{+} 1.75$ & & E 2.77 \\
\hline
\end{tabular}

- Total Disintegration Energy (Mev), or Isotopic Mass

Page 7. 


\begin{tabular}{|c|c|c|c|c|}
\hline Lif-Life & Isotope & $\begin{array}{l}\text { Particles and } \\
\text { Energies (Mev) }\end{array}$ & $\begin{array}{l}\text { Gamma Energies } \\
\frac{(\mathrm{Mev})}{}\end{array}$ & $\begin{array}{l}\text { Total Energy } \\
\text { or Mass }\end{array}$ \\
\hline $1.1 \mathrm{~m}$ & $\mathrm{Cs}^{140}$ & $\beta^{-}$ & $s$ & \\
\hline $1.1 \mathrm{~m}$ & Mo 91 & $\beta^{+} 2.45,2.78,3.99$ & $\begin{array}{l}\text { I.T. } .65 \\
\text { r } 1.54,1.21\end{array}$ & \\
\hline $1.15 \mathrm{~m}$ & $S_{e}^{83}$ & $\beta^{-} 3.4$ & & \\
\hline $1.18 \mathrm{~m}$ & $\mathrm{Pe}^{234}$ & $\beta^{-2 \cdot 31, \cdots}$ & $\begin{array}{l}\text { I.T. } \\
Y .23-1.8\end{array}$ & \\
\hline $1.20 \mathrm{~m}$ & $\operatorname{In}^{114}$ & $\begin{array}{l}\beta^{-} 1.98, \cdots \\
\beta^{+} \sim 1\end{array}$ & $\begin{array}{l}K \\
Y .55-1.28\end{array}$ & $\begin{array}{l}E^{-} 1.98 \\
E^{+} 2.3\end{array}$ \\
\hline $1.20 \mathrm{~m}$ & $0^{14}$ & $\beta^{+} 1.83$ & $r 2.30$ & E 5.15 \\
\hline $1.25 \mathrm{~m}$ & $\mathrm{Rb}^{82}$ & $\beta^{+} 3.2$ & & \\
\hline $1.25 \mathrm{~m}$ & $\mathrm{Xe}^{127}$ & & $\begin{array}{l}\text { I.T. } .175 \\
r .125\end{array}$ & \\
\hline $1.25 \mathrm{~m}$ & $A g^{111}$ & 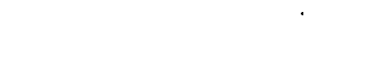 & I.T. & \\
\hline $1.3 \mathrm{~m}$ & $U^{227}$ & $\alpha 6.8$ & & M 227.102 \\
\hline $1.3 \mathrm{~m}$ & Dy 165 & $\beta^{-} \sim 1$ & $\begin{array}{l}\text { I.T. } .108 \\
\gamma .36, .16, .52\end{array}$ & \\
\hline $1.3 \mathrm{~m}$ & $\mathrm{Sb}^{124}$ & $\begin{array}{l}\beta^{-} 3 \\
e^{-}\end{array}$ & I.T. .012 & \\
\hline $1.32 \mathrm{~m}$ & $\mathrm{~T}^{210}$ & $\beta^{-} 1.9$ & $r$ & E 5.4 \\
\hline$\sim 1.33 \mathrm{~m}$ & $\mathrm{Rb}^{92}$ & $\beta^{-}$ & & \\
\hline $1.35 \mathrm{~m}$ & $\mathrm{Cu}^{59}$ & $\beta^{+} 3.7$ & $r .9,1.3$ & \\
\hline $1.4 \mathrm{~m}$ & $\operatorname{Ir}{ }^{192}$ & $\begin{array}{l}e^{-} \\
\beta^{-}\end{array}$ & I.T. .057 & \\
\hline $1.4 \mathrm{~m}$ & $\mathrm{Cl}^{40}$ & $\beta^{-} \sim 7, \cdots$ & $r 1.46,2.75, \sim 6$ & \\
\hline $1.43 \mathrm{~m}$ & $I^{136}$ & $\beta-6.4,5.0,3.6$ & $r 1.4,2.8$ & E 6.4 \\
\hline $1.5 \mathrm{~m}$ & $2 n^{61}$ & $\beta^{+} 5$ & & \\
\hline $1.5 \mathrm{~m}$ & $\mathrm{Pd}^{113}$ & $\beta^{-}$ & & \\
\hline
\end{tabular}

* Total Disintegration Energy (Mev), or Isotople Mass

Page 8. 


\begin{tabular}{|c|c|c|c|c|}
\hline Half-Life & Isotope & $\begin{array}{l}\text { Particles and } \\
\text { Energies (Mev) }\end{array}$ & $\begin{array}{l}\text { Gamma Energles } \\
(\mathrm{Nev}) \\
\end{array}$ & $\begin{array}{l}\text { Total Energy } \\
\text { or Mass }\end{array}$ \\
\hline $1.6 \mathrm{~m}$ & $\mathrm{Co}^{62}$ & $\beta^{-}$ & $r$ & \\
\hline $1.6 \mathrm{~m}$ & $\mathrm{Cs}^{126}$ & $\beta^{+} 3.8, \cdots$ & $\begin{array}{l}K \\
r \cdot 38\end{array}$ & E 4.8 \\
\hline $1.7 \mathrm{~m}$ & $\mathrm{Bi}<198$ & a 6.2 & & \\
\hline $1.7 \mathrm{~m}$ & At $<203$ & a 6.35 & & \\
\hline $1.7 \mathrm{~m}$ & $w^{185}$ & $e^{-}$ & $\begin{array}{l}\mathrm{I} . \mathrm{T} . \\
\mathrm{r} .13, .16\end{array}$ & . \\
\hline $1.7 \mathrm{~m}$ & $\mathrm{Nm}^{57}$ & $\beta-2.6$ & $r .117, .134, .69, \cdots$ & E 2.7 \\
\hline $1.7 \mathrm{~m}$ & $\mathrm{Sc}^{50}$ & $\beta^{-} \sim 3.5$ & $r 1.6,1.2$ & $E \sim 6.3$ \\
\hline $1.7 \mathrm{~m}$ & $\mathrm{Rb}^{91}$ & $\beta^{-}-4.6$ & $r$ & \\
\hline $1.8 \mathrm{~m}$ & $\mathrm{~Pa}^{226}$ & $\alpha 6.81$ & & M 226.099 \\
\hline$<2 \mathrm{~m}$ & $\mathrm{Mo}^{105}$ & $\beta^{-}$ & & \\
\hline $2 m$ & $\mathrm{Te}^{133}$ & $\beta-1.4,2.4$ & $r .6,1.0$ & E 3.0 \\
\hline $2 \mathrm{~m}$ & $\mathrm{Sb}^{132}$ & $\beta^{-}$ & & \\
\hline $2 \mathrm{~m}$ & $\mathrm{Mn}^{54}$ & $\beta^{-}$or $e^{-}$ & I.T. ? & \\
\hline $2 \mathrm{~m}$ & $\mathrm{Ag}^{114}$ & $\beta^{-}$ & & \\
\hline$\sim 2 \mathrm{~m}$ & $\mathrm{Se}^{84}$ & $\beta^{-}$ & & \\
\hline$\sim 2 \mathrm{~m}$ & $\mathrm{Sr}^{94}$ & $\beta^{-}$ & & \\
\hline $2.0 \mathrm{~m}$ & $v^{53}$ & $\beta-2.50$ & $r 1.00$ & . \\
\hline $2.1 \mathrm{~m}$ & $0^{15}$ & $\beta^{+} 1.7$ & & E 2.7 \\
\hline $2.1 \mathrm{~m}$ & $2 n^{60}$ & & & \\
\hline $2.15 \mathrm{~m}$ & $\mathrm{Bi}^{211}$ & $\begin{array}{l}a 6.62,6.27,(9) \\
\beta^{-} \\
e^{-}\end{array}$ & $r .35$ & E. .63 \\
\hline $2.2 \mathrm{~m}$ & $\mathrm{Ac}^{223}$ & a 6.64 & K & M 223.089 \\
\hline
\end{tabular}

* Total Disintegration Energy (Mov), or Isotopic Mass

Page 9. 


\begin{tabular}{|c|c|c|c|c|c|}
\hline Half-Life & Isotope & $\begin{array}{l}\text { Particles and } \\
\text { Energles (Mer) }\end{array}$ & & $\begin{array}{c}\text { Gamna Energles } \\
(\mathrm{MeV}) \\
\end{array}$ & $\begin{array}{l}\text { Total Energy } \\
\text { or Mass * }\end{array}$ \\
\hline $2.2 \mathrm{~m}$ & $\mathrm{TI}^{209}$ & $\beta-1.8,2.3$ & & $\gamma .11-1.6, .12 \mathrm{D}$ & E 3.9 \\
\hline $2.2 \mathrm{~m}$ & $\operatorname{sn} 132$ & $\beta^{-}$ & & & \\
\hline $2.2 \mathrm{~m}$ & $\mathrm{Zn}^{71}$ & $\beta-2.4$ & & $r .51, \cdots$ & E 2.9 \\
\hline $2.3 \mathrm{~m}$ & $\mathrm{Ag}^{108}$ & $\begin{array}{l}\beta-1.77, \cdots \\
\beta+.8\end{array}$ & & $\begin{array}{l}K \\
r .62, .43, .60\end{array}$ & $\begin{array}{l}\mathrm{E}^{-} 1.77 \\
\mathrm{E}^{+} 1.8\end{array}$ \\
\hline $2.30 \mathrm{~m}$ & $A^{28}$ & $\beta-2.87$ & & $r 1.78$ & E 4.65 \\
\hline $2.4 \mathrm{~m}$ & $\operatorname{Re}^{180}$ & $\beta^{+} 1.1$ & & $r .11, .88$ & \\
\hline $2.5 \mathrm{~m}$ & $p^{30}$ & $\beta^{+} 3.3$ & & & E 4.3 \\
\hline $2.5 \mathrm{~m}$ & $G_{e}^{64}$ & $\beta^{+} \sim 5$ & & $r .97-3.8$ & E 7 \\
\hline $2.5 \mathrm{~m}$ & $\mathrm{NB}^{99}$ & $\beta^{-} 3.2$ & & & E 3.2 \\
\hline $2.6 \mathrm{~m}$ & $\mathrm{Sn}^{130}$ & $\beta^{-}$ & & & \\
\hline $2.60 \mathrm{~m}$ & $\mathrm{Ba}^{137}$ & $\theta^{-}$ & & I.T. .662 & \\
\hline $2.7 \mathrm{~m}$ & $\mathrm{Ra}^{213}$ & a 6.90 & & & \\
\hline $2.7 \mathrm{~m}$ & $a b^{90}$ & $\beta-5.7$ & & $r$ & \\
\hline $3 \mathrm{~m}$ & $\operatorname{Em}^{2043}$ & a 6.3 & & & \\
\hline $3 \mathrm{~m}$ & $R_{0} 190$ & $\beta-1.7$ & & $\begin{array}{l}r .19, .36, .56, \\
.40 .80\end{array}$ & \\
\hline $3.0 \mathrm{~m}$ & $\mathrm{Ce}^{\mathrm{K}}$ & $\beta^{-} 2.0$ & & $r$ & \\
\hline $3.0 \mathrm{~m}$ & $\mathrm{Br}^{85}$ & $\beta-2.5$ & & no $r$ & E 2.8 \\
\hline $3.05 \mathrm{~m}$ & $P_{0}^{218}$ & $\beta^{-} 6.00$ & & & E 218.077 \\
\hline $3.1 \mathrm{~m}$ & $\mathrm{TI}^{208}$ & $\beta^{-} 1.79,1.28$ & $\bullet$ & $\begin{array}{l}r 2.615, .580, .51, \\
\quad .86, . .\end{array}$ & E 4.99 \\
\hline $3.2 \mathrm{~m}$ & $K r^{89}$ & $\beta^{-} 4.2,2$ & & $r$ & \\
\hline $3.4 \mathrm{~m}$ & $\mathrm{Sn}^{131}$ & $\beta^{-}$ & & & \\
\hline $3.4 \mathrm{~m}$ & $\operatorname{Pr}^{140}$ & $\beta^{+2.3}$ & & $K$ & E 3.3 \\
\hline
\end{tabular}

- Total Disintegration Energy (Mov), or Isotopic Mass 


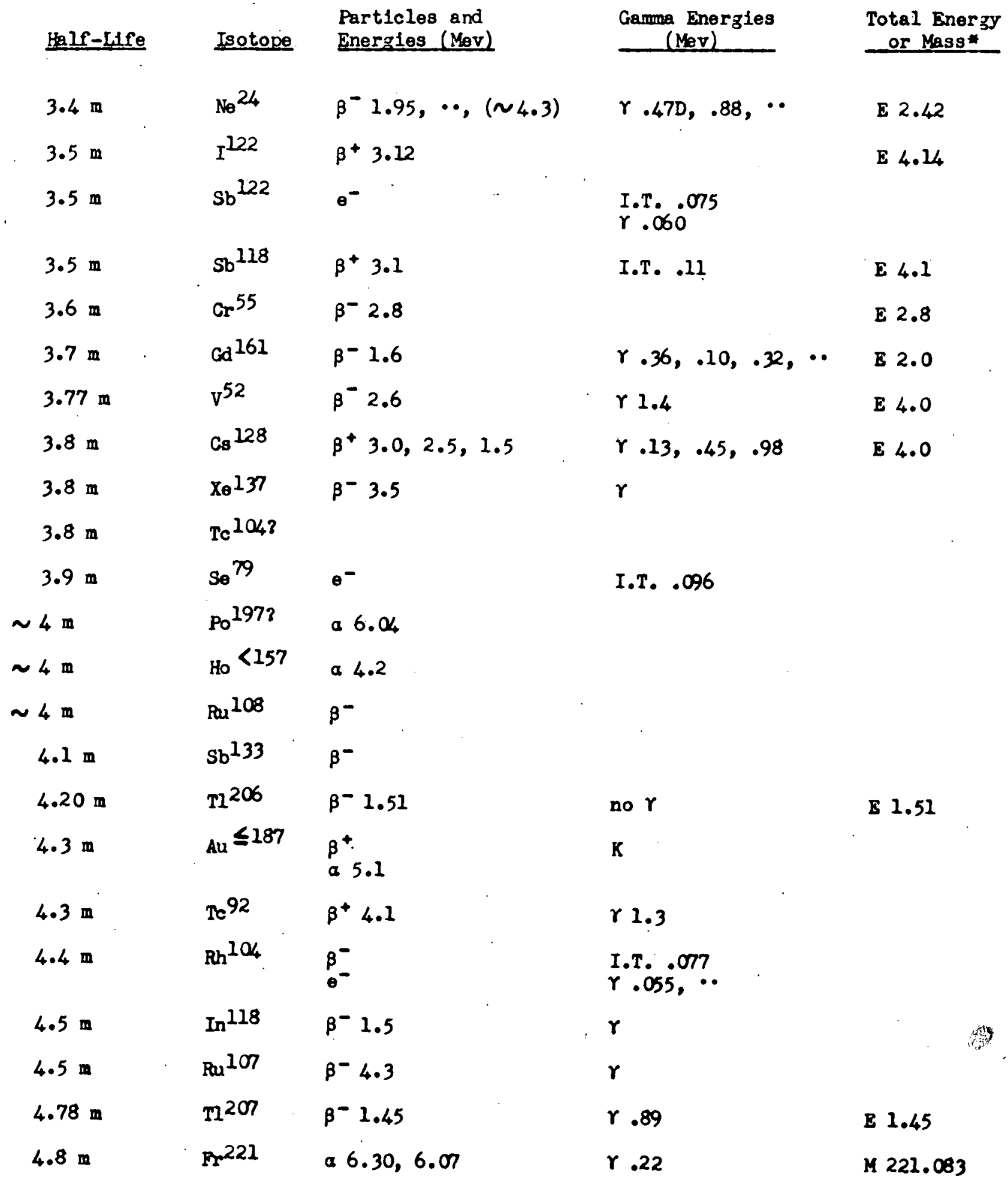

* Total Disintegration Energy (Mev), or Isotopic Mass 


\begin{tabular}{|c|c|c|c|c|}
\hline Hiff-Lfe & Isotope & $\begin{array}{l}\text { Particles and } \\
\text { Energies (Mev) }\end{array}$ & $\begin{array}{l}\text { Gamma Energles } \\
\text { (Mev) } \\
\end{array}$ & $\begin{array}{l}\text { Total } \mathrm{Er} \\
\text { or Mas }\end{array}$ \\
\hline $4.8 \mathrm{~m}$ & $P^{109}$ & $e^{-}$ & I.T. . .17 & \\
\hline $4.9 \mathrm{~m}$ & $\mathrm{Cd}^{11 \mathrm{II}}$ & $e^{-}$ & $\begin{array}{l}\text { I.T. } .150 \\
r .247 \mathrm{D}\end{array}$ & \\
\hline$<5 \mathrm{~m}$ & $\mathrm{RB}^{229}$ & $\beta^{-}$ & & \\
\hline$<5 \mathrm{~m}$ & $\operatorname{Th}^{235}$ & $\beta^{-}$ & & \\
\hline $5.0 \mathrm{~m}$ & $s^{37}$ & $\beta^{-1.6,4.7}$ & $r 3.1$ & E 4.7 \\
\hline $5.1 \mathrm{~m}$ & $\mathrm{Cu}^{66}$ & $\beta^{-2.63,1.59}$ & $r 1 . \alpha_{4}$ & E 2.63 \\
\hline $5.2 \mathrm{~m}$ & $\mathrm{Hg}^{205}$ & $\beta^{-} 1.6,1.4$ & $\gamma .20$ & E 1.6 \\
\hline $5.5 \mathrm{~m}$ & $\mathrm{Fe}^{61}$ & $\beta^{-}$ & $r$ & \\
\hline $5.80 \mathrm{~m}$ & $\mathrm{Ti}^{51}$ & $\beta^{-} 2.1,1.5$ & $r .32, .93, .61$ & E 2.4 \\
\hline$<6 m$ & $\mathrm{Br}^{78}$ & $\beta+2.4$ & & E 3.4 \\
\hline $6 \mathrm{~m}$ & $\mathrm{Ba}^{142}$ & $\beta^{-}$ & & \\
\hline $6 \mathrm{~m}$ & $\mathrm{Cs}^{123}$ & $\beta^{+}$ & & \\
\hline$\sim 6 \mathrm{~m}$ & Bo 1987 & a 5.94 & & \\
\hline $6.4 \mathrm{~m}$ & $\mathrm{~B} r^{78}$ & & $\begin{array}{l}\text { I.T. } \\
r .05, .11\end{array}$ & \\
\hline $6.5 \mathrm{~m}$ & $2 a^{1344}$ & $\beta^{+} 2.7$ & $\mathbf{K}$ & E 3.7 \\
\hline $6.6 \mathrm{~m}$ & $\mathrm{Mb}^{94}$ & $\begin{array}{l}\beta^{-}-1.3 \\
\theta^{-}\end{array}$ & $\underset{r}{\text { I.T. }} \cdot . \alpha_{4}$ & \\
\hline $6.6 \mathrm{~m}$ & $\mathrm{Al}^{29}$ & $\beta-2.5,1.4$ & $r 1.28,2.43$ & E 3.8 \\
\hline $7 \mathrm{~m}$ & $\operatorname{Bm}^{206}$ & $a \cdot 6.22$ & $\mathbf{K}$ & \\
\hline $7 \mathrm{~m}$ & $D_{y} \leqq 153$ & a 4.2 & & \\
\hline $7 \mathrm{~m}$ & Ir 197 & $\beta-1.6, \cdots$ & $r 1.8$ & \\
\hline $7 \mathrm{~m}$ & $A t^{203}$ & $\alpha 6.10$ & & \\
\hline $7 \mathrm{~m}$ & ${ }_{B 1}^{198}$ & a 5.83 & $\mathbf{K}$ & \\
\hline
\end{tabular}

* Total Disintegration Energy (Mov), or Isotopic Mass

Page 12. 


\begin{tabular}{|c|c|c|c|c|}
\hline 暨f-Lfe & Isotope & $\begin{array}{l}\text { Particles and } \\
\text { Energles (Mov) }\end{array}$ & $\begin{array}{l}\text { Gamma Energles } \\
\frac{(\mathrm{Mev})}{}\end{array}$ & $\begin{array}{l}\text { Total Energy } \\
\text { or Mass: } \\
\end{array}$ \\
\hline $7 \mathrm{~m}$ & $\mathrm{Sr}^{93}$ & $\beta^{-}$ & & \\
\hline$\sim 7 \mathrm{~m}$ & $A^{68}$ & $\beta^{+}$ & & \\
\hline $7.3 \mathrm{~m}$ & $E^{247}$ & a 7.35 & $K(3)$ & M 247.151 \\
\hline $7.3 \mathrm{~m}$ & $\mathrm{~Np}^{240}$ & $\beta-2.16, \cdots$ & $r .56, .91,1.4$ & E 2.16 . \\
\hline $7.7 \mathrm{~m}$ & $K^{-38}$ & $\beta+2.7$ & $r 2.1$ & E 5.8 \\
\hline $8 \mathrm{~m}$ & $\mathrm{Bi}^{215}$ & $\beta^{-}$ & & M 215.069 \\
\hline $8 \mathrm{~m}$ & $\sin ^{143}$ & $\beta^{+} 2.3$ & & E 3.3 \\
\hline $8 \mathrm{~m}$ & $\mathrm{Th}^{225}$ & a $6: 57$ & K & M 225.094 \\
\hline $8 \mathrm{~m}$ & $\mathrm{Ga}^{65}$ & $\beta^{+} 2.2$ & & E 3.2 \\
\hline$\sim 8 \mathrm{~m}$ & $P^{200}$ & a 5.77 & $\mathrm{~K}$ & \\
\hline $8.7 \mathrm{~m}$ & $\mathrm{Ca}^{49}$ & $\beta^{-} 2.0,1.0$ & $r 3.09,4.05,4.7$ & E 5.1 \\
\hline$<9 m$ & $\mathrm{Em}^{216}$ & a 8.02 & & M 216.068 \\
\hline$<9 \mathrm{~m}$ & $\mathrm{Th}^{224}$ & a 7.13 & & M 224.091 \\
\hline$<9 m$ & $P^{220}$ & a 7.43 & & M 220.080 \\
\hline $9 \mathrm{~m}$ & LA 136 & $\beta^{+} 2.1$ & K & \\
\hline $9 \mathrm{~m}$ & $0 s 190$ & & $\begin{array}{l}\text { I.T. } . .62 \\
r .19-.56\end{array}$ & \\
\hline $9 \mathrm{~m}$ & $R h^{98}$ & $\beta^{+} 3.3$ & $r .65, \cdots$ & \\
\hline $9 \mathrm{~m}$ & 4879 & $\beta-2.3$ & $(r .096)$ & E 2.4 \\
\hline $9 m$ & $\mathrm{Fe}^{53}$ & $\beta^{+} 2.6$ & $r \cdot 37$ & E 3.6 \\
\hline $9.3 \mathrm{~m}$ & $\mathrm{Ta}^{178}$ & $\begin{array}{l}\beta^{+} 1.1 \\
e^{-} .08\end{array}$ & $\begin{array}{l}K \\
r \sim 1.5\end{array}$ & \\
\hline $9.3 \mathrm{~m}$ & $U^{228}$ & a 6.67 & $\mathbf{K}$ & M 228.103 \\
\hline $9.5 \mathrm{~m}$ & $\mathrm{Cs}^{139}$ & $\beta^{-} \sim 4$ & $\boldsymbol{r}$ & \\
\hline $9.5 \mathrm{~m}$ & $\mathrm{Sn}^{125}$ & $\beta-2.1, \cdots$ & $r .33,1.4$ & E 2.4 \\
\hline
\end{tabular}

Page 13. 


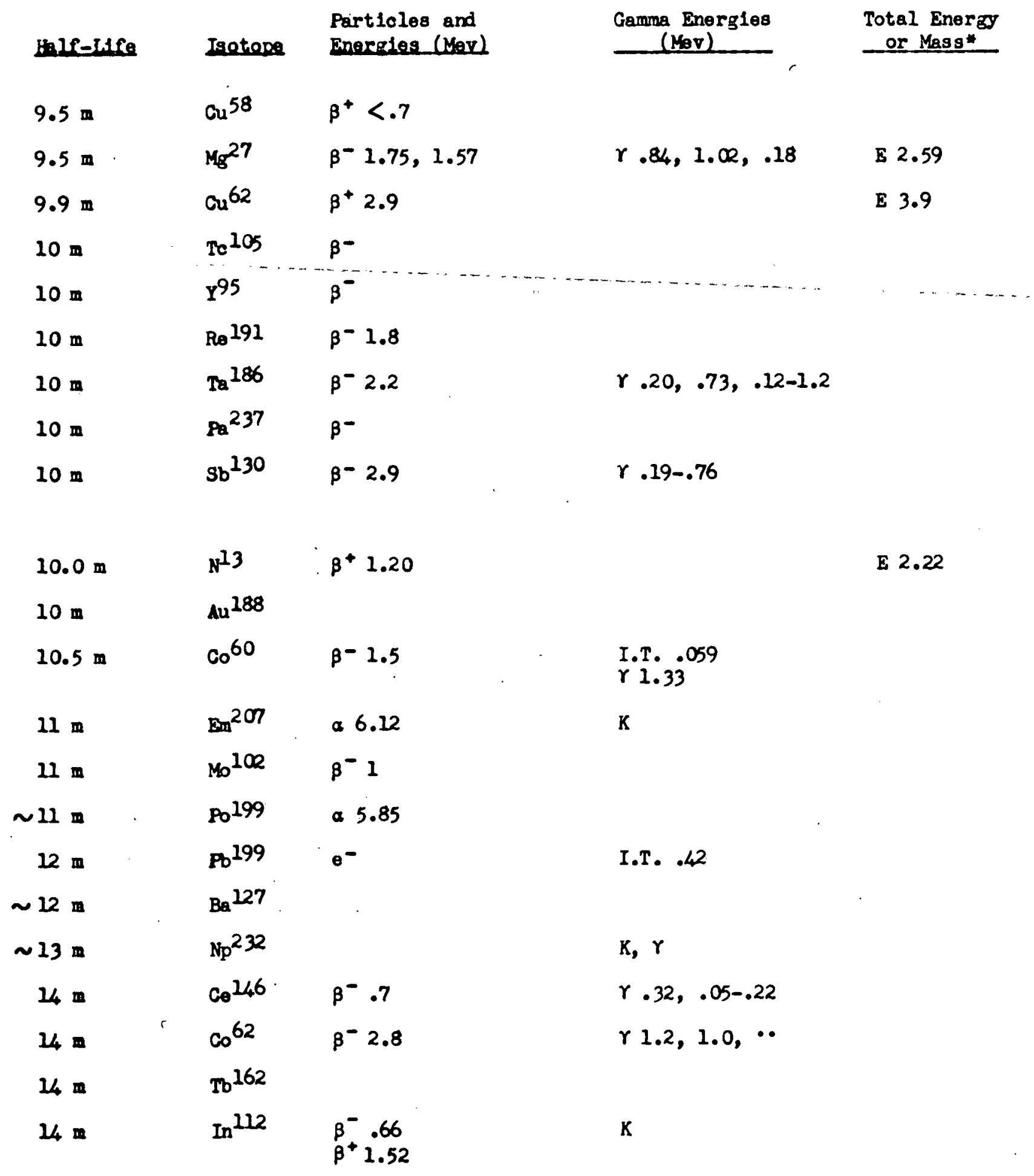

* Total Disintegration Energy (Mev), or Isotopic Mass

Page 14 . 


\begin{tabular}{|c|c|c|c|c|}
\hline 些任-Lfe & Isotope & $\begin{array}{l}\text { Particles and } \\
\text { Energies (Mev) }\end{array}$ & $\begin{array}{l}\text { Gamma Energies } \\
\text { (Mev) }\end{array}$ & $\begin{array}{l}\text { Total Ener By } \\
\text { or Nasa } \\
\end{array}$ \\
\hline $14 \mathrm{~m}$ & $\operatorname{In} 112$ & $\beta^{-} .66$ & $\mathbf{K}$ & E 1.52 \\
\hline $\mathbf{u}_{4} \mathrm{~m}$ & $\mathrm{Rb}^{91}$ & $\beta=3.0$ & $r$ & \\
\hline $15 \mathrm{~m}$ & $\mathrm{Sb}^{116}$ & $\beta+2.4, \cdots$ & $r 1.27, .9,2.2$ & E 4.7 \\
\hline $15 \mathrm{~m}$ & $A^{69}$ & $\beta+2.9$ & $r .23$ & \\
\hline $15 \mathrm{~m}$ & $\mathrm{Nd} 151$ & $\beta-1.9$ & $r_{i .1}^{.08, .11, .42, .7}$ & E 2.0 \\
\hline $15 \mathrm{mb}$ & $x_{\theta} 135$ & $\theta^{-}$ & I.T. . .52 & \\
\hline $15 \mathrm{~m}$ & $\mathrm{Ga}^{65}$ & $\beta+2.5$ & $\begin{array}{l}\text { I.T. } .052 \\
r .09, .11\end{array}$ & \\
\hline $15 \mathrm{~m}$ & $T^{101}$ & $\beta-1.2$ & $r \cdot 30$ & B 1.5 \\
\hline $15 \mathrm{~m}$ & Mol01 & $\beta^{-} 1.2,2.2$ & $r .19, .96$ & $E 2.4$ \\
\hline $15 \mathrm{~m}$ & $F^{222}$ & $\begin{array}{l}\beta^{-} \\
\alpha\end{array}$ & . & \\
\hline $15 \mathrm{~m}$ & $\mathrm{Rb}^{89}$ & $\beta-3.9, \ldots$ & $\begin{array}{l}r 1.05, .25, .66, \\
1.26-2.20\end{array}$ & E 3.9 \\
\hline$\sim 15 \mathrm{~m}$ & $\mathrm{Au}^{-187}$ & & 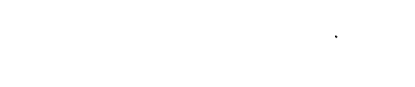 & \\
\hline $15.6 \mathrm{~m}$ & $\mathrm{Mo}^{91}$ & $\beta^{+} 3.44$ & & E 4.46 \\
\hline $16 m$ & $\Delta \mathrm{g}^{102}$ & $\beta^{+}$ & & \\
\hline $16 \mathrm{~m}$ & $\mathrm{Ta}^{182}$ & $\mathrm{e}^{-}$ & I.T. . .18 & \\
\hline $17 \mathrm{~m}$ & $\operatorname{Pr}^{144}$ & $\beta^{-2.98, .8,2.3}$ & $r .70,2.18,1.48$ & E 2.98 \\
\hline $17 \mathrm{~m}$ & $X_{\theta}^{138}$ & $\beta-2.4$ & $r .42, .51,1.8,2.0$ & \\
\hline $17 \mathrm{~m}$ & $\mathrm{Fd}^{98}$ & $\beta^{+}$ & & \\
\hline $17 \mathrm{~m}$ & $\mathrm{Sb} 120$ & $\beta^{+} 1.70$ & $r 1.18$ & E 2.72 \\
\hline $17 \mathrm{~m}$ & $Y^{94}$ & $\beta^{-} .5 .4$ & $r 1.4$ & E $5.4(3)$ \\
\hline
\end{tabular}

- Total Disintegration Energy (Mev), or Isotopic Mass

Page 15. 


\begin{tabular}{|c|c|c|c|c|}
\hline Lalf-L1fe & Isotope & $\begin{array}{l}\text { Particles and } \\
\text { Energies (Mev) }\end{array}$ & $\begin{array}{l}\text { Gamma Energies } \\
\frac{(\mathrm{Mev})}{}\end{array}$ & $\begin{array}{l}\text { Total En } \\
\text { or Mas } \\
\end{array}$ \\
\hline $18 \mathrm{~m}$ & $5 e^{81}$ & $\beta^{-1.38}$ & & E 1.38 \\
\hline $18 \mathrm{~m}$ & In 119 & $\beta^{-} 2.7$ & no $\gamma$ & E 2.7 \\
\hline $18 \mathrm{~m}$ & $I^{119}$ & $\beta^{+}$ & & \\
\hline $18 \mathrm{~m}$ & $\mathrm{Br}{ }^{80}$ & $\begin{array}{l}\beta^{-2} 2.0,1.4 \\
\beta^{+} .86\end{array}$ & $\begin{array}{l}K \\
r .62\end{array}$ & $\begin{array}{l}E^{-} 2.0 \\
E^{+} 1.9\end{array}$ \\
\hline $18 m$ & $\mathrm{rb}^{167}$ & & $\begin{array}{l}K \\
r .118, .18 ?, .33 ?\end{array}$ & \\
\hline $18 \mathrm{~m}$ & $\mathrm{Ba}^{142}$ & $\beta^{-} 2.8$ & $r$ & \\
\hline $18 \mathrm{~m}$ & Burlut & $\beta^{+} 2.4$ & & \\
\hline $18 \mathrm{n}$ & $9 n^{109}$ & $\beta^{+}$ & $\begin{array}{l}K \\
r .073, .68\end{array}$ & \\
\hline $18 \mathrm{~m}$ & $\mathrm{PO}^{201}$ & a 5.67 & K & \\
\hline $18 \mathrm{~m}$ & $R b^{88}$ & $\beta^{-} 5.2,3.3,2$ & $\begin{array}{l}r 1.85, .91,2.7 \\
1.39-4.9\end{array}$ & E 5.2 \\
\hline $19=$ & $\operatorname{Tn} 172$ & $\beta^{-}$ & & \\
\hline $19 \mathrm{~m}$ & $\mathrm{Fr}^{212}$ & a $6.39,6.41,6.34$ & $\mathrm{~K}$ & \\
\hline $19 \mathrm{~m}$ & $\mathrm{Ge}^{67}$ & $\beta^{+} 3.4$ & $r .17$ & \\
\hline $19 \mathrm{~m}$ & $D y \leqq 153$ & a 4.1 & 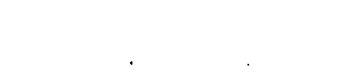 & \\
\hline $19 \mathrm{~m}$ & $\operatorname{Ir} ?$ & & $k$ & \\
\hline$\sim 19 \mathrm{~m}$ & $\mathrm{La}^{1 / 3}$ & $\beta^{-}$ & & \\
\hline $19.7 \mathrm{~m}$ & $\mathrm{BI}^{2 \mathrm{U}}$ & $\begin{array}{l}\beta^{-} 1.6,3.17, \cdots \\
\text { a } 5.5, \cdots,(8.3-10.5)\end{array}$ & $\begin{array}{l}r .61,1.12,1.76 \text {, } \\
.45-2.43\end{array}$ & E 3.17 \\
\hline $20 m$ & $\mathrm{Pm}^{1 / 41}$ & $\beta^{+} \sim 2.6$ & & \\
\hline $20 \mathrm{~m}$ & $\mathrm{Re}^{188}$ & $0^{-}$ & $\mathrm{I.T}_{\mathrm{r} .06} \cdot 10$ & \\
\hline $20 \mathrm{~m}$ & $\operatorname{Eu} 159$ & $\beta^{-}$ & & \\
\hline $20.5 \mathrm{~m}$ & $\mathrm{c}^{11}$ & $\beta^{+} .96$ & no $r$ & E 1.98 \\
\hline
\end{tabular}

- Total Disintegration Energy (Mev), or Isotopic Mass

Page 16. 


\begin{tabular}{|c|c|c|c|c|}
\hline Helf-Lfe & Isotope & $\begin{array}{l}\text { Particles and } \\
\text { Energies (Mov) }\end{array}$ & $\begin{array}{l}\text { Ge ung Energies } \\
\frac{(\mathrm{Mev})}{}\end{array}$ & $\begin{array}{l}\text { Totel EnersJ } \\
\text { or Mass* }\end{array}$ \\
\hline $21 \mathrm{~m}$ & $\mathrm{Rb}^{84}$ & & $\begin{array}{l}\mathrm{I} . \mathrm{T} \cdot .23, .46 \\
\mathrm{~K} \\
\mathrm{r} .23, .89\end{array}$ & \\
\hline $21 \mathrm{~m}$ & In 112 & $e^{-}$ & $\underset{(\gamma . T .}{\mathrm{I} .15)}$ & \\
\hline $21 \mathrm{~m}$ & $\mathrm{Ga}^{70}$ & $\beta^{-} 1.65, \cdots$ & $\gamma 1 . \alpha_{4}, .17$ & E 1.65 \\
\hline $21 \mathrm{~m}$ & $\mathrm{Ag}^{115}$ & $8^{-} 3$ & $r .23$ & E 3 \\
\hline $21 \mathrm{~m}$ & $M^{52}$ & $\beta^{+} 2.7$ & $\underset{r}{1 . T .4} .39$ & \\
\hline $21 \mathrm{~m}$ & $\mathrm{Sb}^{124}$ & $\begin{array}{l}\beta^{-} \\
e^{-}\end{array}$ & I.T. .018 & \\
\hline $22 \mathrm{~m}$ & $\mathrm{Nd}^{138}$ & $\begin{array}{l}\beta^{+} 2.4 \\
\theta-(?)\end{array}$ & & $\cdot$ \\
\hline $22 \mathrm{~m}$ & $\mathrm{Sb}^{131}$ & $\beta^{-} 1.1$ & & \\
\hline $22 \mathrm{~m}$ & $\operatorname{Pr} 135$ & $\beta^{+} 2.5$ & $r .30, .22, .08$ & \\
\hline $22 \mathrm{~m}$ & $\mathrm{Rh}^{107}$ & $\beta^{-} 1.2, \sim 2$ & $r .31, .40, .1-.7$ & \\
\hline $22 \mathrm{~m}$ & $K^{44}$ & $\beta^{-} 4.9,1.5$ & $r 1.1,2.1,2.5, \ldots$ & \\
\hline $22 \mathrm{~m}$ & $\mathrm{Fr}^{223}$ & $\begin{array}{l}\beta^{-} 1.0,1.3 \\
\alpha 5.3\end{array}$ & $r .050-.31$ & M 223.090 \\
\hline $22 \mathrm{~m}$ & $\mathrm{Pd}^{112}$ & $\beta^{-} 2.14, \cdots$ & $r .38-.73$ & \\
\hline $22 \mathrm{~m}$ & Lu178 & $\beta^{-}$ & & \\
\hline $22 \mathrm{~m}$ & Ho 162 & $\begin{array}{l}\beta^{+} 1.3 \\
\theta^{-} .17\end{array}$ & $\begin{array}{l}K \\
r \sim 1.2\end{array}$ & \\
\hline $23 \cdot \mathrm{m}$ & $\min ^{208}$ & a $6 . u_{4}$ & K & \\
\hline $23 \mathrm{~m}$ & $\mathrm{Em}^{212}$ & a 6.26 & & M 212.057 \\
\hline $23 \mathrm{~m}$ & $\mathrm{Sm}^{155}$ & $\beta-1.8$ & $\gamma .25, .105$ & E 2.2 \\
\hline $23.3 \mathrm{~m}$ & $\mathrm{Th}^{233}$ & $\beta^{-} 1.23, \cdots$ & $r .098-.66$ & E 1.23 \\
\hline $23.5 \mathrm{~m}$ & $U^{239}$ & $\beta-1.21$ & $r .074$ & E 1.28 \\
\hline
\end{tabular}

- Total Disintegration Energy (Mev), or Isotopic Mass

Page 17. 


\begin{tabular}{|c|c|c|c|c|}
\hline Hif-Life & Isotope & $\begin{array}{l}\text { Particles and } \\
\text { Energies (Mov) }\end{array}$ & $\begin{array}{l}\text { Gamma Energies } \\
\text { (Mev) }\end{array}$ & $\begin{array}{l}\text { Total Ener } 35 \\
\text { or Mass" }\end{array}$ \\
\hline $24 \pi$ & $P \mathbf{P}^{99}$ & & $\gamma .13$ & \\
\hline $24 \mathrm{~m}$ & $\operatorname{Pr}^{146}$ & $\beta^{-} 3.7,2.3$ & $r .46,1.49, .75, \cdots$ & E 4.2 \\
\hline $24 \mathrm{~m}$ & $\mathrm{Cu}^{60}$ & $\beta^{+} 2.0,3.0,3.9$ & $\begin{array}{l}r 1.33,1.76, .85 \\
2.1-4.0\end{array}$ & E 6.3 \\
\hline $24 n$ & $A B^{106}$ & $\beta^{+} 1.96,1.45$ & $\begin{array}{l}k \\
\gamma .5 i\end{array}$ & E 2.98 \\
\hline $24 m$ & $\mathrm{~Pa}^{235}$ & $\beta^{-} 1.4$ & no $r$ & E 1.4 \\
\hline$<24=$ & $P O^{217}$ & a 6.5 & & \\
\hline $25 \mathrm{n}$ & $\operatorname{Bin}^{221}$ & $\beta^{-}$ & & \\
\hline $25 \mathrm{n}$ & $\mathrm{To}^{131}$ & $8-2.1, \cdots$ & $r .05-.77$ & E 2.3 \\
\hline $25 \mathrm{a}$ & $\cos ^{244}$ & a 7.17 & $K(?)$ & M 244.142 \\
\hline $25 m$ & $\Delta t^{205}$ & a 5.90 & $\mathbf{K}$ & \\
\hline $25 \mathrm{~m}$ & $\operatorname{sim}^{2 / 46}$ & $\beta^{-1.22, \cdots}$ & $\begin{array}{l}r 1.07, .80, .10 \\
.018, . .\end{array}$ & E 2.4 \\
\hline $25 \mathrm{n}$ & $S 0^{83}$ & $\beta^{-} 1.5$ & $\gamma .04, .06, .18, .95$ & \\
\hline $25 n$ & $\mathrm{Hg}^{189}$ & $\beta^{+}$ & $\begin{array}{l}K \\
\gamma .029\end{array}$ & \\
\hline$\sim 25 \mathrm{~m}$ & $B 1199$ & a 5.47 & $\mathbf{K}$ & \\
\hline$\sim 25 \mathrm{~m}$ & At $2 \alpha_{4}$ & . & $\mathbf{K}$ & \\
\hline $25.0 \mathrm{~m}$ & $I^{128}$ & $\beta^{-} 2.12,1.67, \ldots$ & 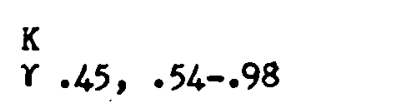 & $E^{-2.12}$ \\
\hline $26 \mathrm{~m}$ & $P u^{2} 35$ & a 5.85 & $L, K$ & M 235.119 \\
\hline $26 \mathrm{~m}$ & $\mathrm{Am}^{2444}$ & $\begin{array}{l}\beta^{-}-1.5 \\
\theta-1.5\end{array}$ & $\mathrm{~K}$ & M 244.141 \\
\hline $26 \mathrm{~m}$ & $\mathrm{Au}^{201}$ & $\beta-1.5$ & $\gamma .55$ & E 1.5 \\
\hline $26.8 \mathrm{~m}$ & $\mathrm{~Pb}^{214}$ & $\beta^{-} .7, \cdots$ & $\begin{array}{l}r .3520, .2952, \\
.053-.259\end{array}$ & E 1.0 \\
\hline
\end{tabular}

- Total Disintegration Energy (Mov), or Isotopic Mass

Page 18. 


\begin{tabular}{|c|c|c|c|c|}
\hline belf-ufe & Isetope & $\begin{array}{l}\text { Particles and } \\
\text { Enerzles (Mer) }\end{array}$ & $\begin{array}{l}\text { Gamme Energites } \\
\text { (Mev) }\end{array}$ & $\begin{array}{l}\text { Total Enerzy } \\
\text { or Nass }\end{array}$ \\
\hline $27 \mathrm{~m}$ & $A g^{104}$ & $\beta^{+} 2.70$ & $r .55, .12, \cdots$ & \\
\hline $29 \mathrm{~m}$ & $3 r^{81}$ & $\beta^{+}$ & & \\
\hline $30 \mathrm{~m}$ & $\mathrm{Cs}^{130}$ & $\begin{array}{l}\beta^{+} 1.97 \\
\beta^{-} .44\end{array}$ & $K$ & $\begin{array}{l}E^{-} .44 \\
E^{+} 2.99\end{array}$ \\
\hline $30 \mathrm{~m}$ & $w^{179}$ & & $\mathrm{~K}$ & \\
\hline $30 \mathrm{~m}$ & $\mathrm{Em}^{209}$ & $a \cdot 6 \cdot \alpha_{4}$ & $K$ & 1 \\
\hline $30 \mathrm{~m}$ & $P_{t} 199$ & $\beta^{-} \sim 1.2$ & $r .07-.96$ & $E \geq 1.7$ \\
\hline $30 \mathrm{~m}$ & $\mathrm{Fm}^{250}$ & a 7.43 & & M 250.158 \\
\hline $30 \mathrm{~m}$ & $\operatorname{In} 107$ & $\beta+2$ & $\gamma$ & \\
\hline $30 \mathrm{~m}$ & $\mathrm{Nb} 98(3)$ & $\beta^{-}$ & & \\
\hline$\sim 30 \mathrm{~m}$ & $\mathrm{Cd}^{118}$ & $(\beta-4)$ & & \\
\hline $31 \mathrm{~m}$ & $\mathrm{Th}^{226}$ & $a 6.34,6.23,6.10, \cdots$ & $r .11, .24, .13, .19$ & M 226.096 \\
\hline $31 \mathrm{~m}$ & $v^{47}$ & $\beta^{+} 1.89$ & & E 2.89 \\
\hline $32 \mathrm{~m}$ & $\mathrm{Cs} 138$ & $\beta-3.40, \cdots$ & $\begin{array}{l}r 1.43,1.0, .46 \\
2.2, .13-3.3\end{array}$ & \\
\hline $32 \mathrm{~m}$ & $\mathrm{Br}^{84}$ & $\beta^{-} 4.68, \cdots$ & $r .89,1.9$ & \\
\hline $32.4 \mathrm{~m}$ & $\mathrm{Cl}^{34}$ & $\beta+2.5,1.4$ & $\underset{r . T .1}{I .14}, 1.1,3.2, \cdots$ & \\
\hline $34 \mathrm{~m}$ & $K^{45}$ & $\beta^{-}$ & & \\
\hline $35 \mathrm{~m}$ & $S n^{111}$ & $\beta^{+} 1.51$ & $K$ & E 2.53 \\
\hline $35 \mathrm{~m}$ & $\mathrm{~Np}^{233}$ & $\begin{array}{l}a .53 \\
e^{-} . .3\end{array}$ & $\begin{array}{l}K \\
\gamma\end{array}$ & M 233.114 \\
\hline $35 \mathrm{~m}$ & $\mathrm{Bi}^{200}$ & & $K$ & \\
\hline $35 \mathrm{~m}$ & $R h 97$ & $\beta^{+}$ & & \\
\hline $36 \mathrm{~m}$ & $\mathrm{Br}^{74}$ & $\beta^{+}$ & $\mathbf{K}$ & \\
\hline $36 \mathrm{~m}$ & $\mathrm{Pu}^{232}$ & a 6.58 & $K$ & M 232.114 \\
\hline
\end{tabular}

- Total Disintegration Energy (Mev), or Isotopic Mass

Page 19. 


\begin{tabular}{|c|c|c|c|c|}
\hline Half-Lfe & Isotope & $\begin{array}{l}\text { Particles and } \\
\text { Energies (Mev) }\end{array}$ & $\begin{array}{c}\text { Gamma Energies } \\
\text { (MeV) }\end{array}$ & $\begin{array}{l}\text { Total Energy } \\
\text { or Mass * } \\
\end{array}$ \\
\hline $36.1 \mathrm{~m}$ & $\mathrm{~Pb}^{211}$ & $\beta^{-} 1.4, .5$ & $r .83, .06-.76$ & E 1.4 \\
\hline $37 \mathrm{~m}$ & $\mathrm{Ho}^{164}$ & $\beta^{-} .99, .90$ & $\begin{array}{l}K \\
\text { I.T. (?) .046 } \\
\gamma .073, .0900, .037\end{array}$ & $\mathrm{E}^{-} .99$ \\
\hline $37.3 \mathrm{~m}$ & C1 38 & $\beta^{-} 4.8,1.1,2.7$ & $r 2.1,1.6$ & E 4.8 \\
\hline $38 m$ & $\mathrm{PQ}^{227}$ & a 6.46 & $\mathrm{~K} \cdots \cdots \cdots \cdots$ & M $227.100 \ldots$ \\
\hline $38 \mathrm{~m}$ & $\mathrm{zn}^{63}$ & $\beta^{+} 2,36,1.40, \cdots$ & $\stackrel{K}{r} .96,1.9,2.6$ & E 3.38 \\
\hline $40 \mathrm{~m}$ & $\mathrm{Sb}^{130}$ & $\beta^{-}$ & $r$ & \\
\hline $40 \mathrm{~m}$ & $\sin 123$ & $\beta^{-} 1.26$ & $\gamma .15$ & E 1.41 \\
\hline $40 \mathrm{~m}$ & $x^{121}$ & $\beta^{+}$ & $r .096$ & \\
\hline $41 \mathrm{~m}$ & $\mathrm{Ra}^{227}$ & $\beta-1.30$ & $\gamma .29, .50$ & E 1.30 \\
\hline $42 \mathrm{~m}$ & $P B^{197}$ & & $\begin{array}{l}\mathrm{K} \\
\mathrm{I} . \mathrm{T} .(\mathrm{l}) \\
\mathrm{Y} .17, .22, .23, \\
.32, .39\end{array}$ &.. \\
\hline $42 \mathrm{~m}$ & $\mathrm{Cr}^{49}$ & $\beta^{+} 1.54, \cdots$ & $r .15, .09, .06$ & E 2.56 \\
\hline $42 \mathrm{~m}$ & $\mathrm{Au}^{-189}$ & & $\begin{array}{l}K \\
\gamma .29, .14\end{array}$ & \\
\hline $43 \mathrm{~m}$ & $\mathrm{Hg}^{199}$ & $e^{-}$ & $\begin{array}{l}\text { I.T. } .368 \\
r .158 \mathrm{D}\end{array}$ & \\
\hline $44 \mathrm{~m}$ & $\operatorname{cr}^{245}$ & a 7.11 & K & \\
\hline $44 \mathrm{~m}$ & $\mathrm{Te}^{134}$ & $\beta^{-}$ & & \\
\hline $44 \mathrm{~m}$ & $\mathrm{Te}^{93}$ & $\theta^{-}$ & $\begin{array}{l}\text { I.T. .39 } \\
\mathrm{K} \\
\mathrm{r} 2.7\end{array}$ & \\
\hline $44 \mathrm{~m}$ & $\mathrm{Se}^{70}$ & $\beta^{+}$ & & \\
\hline $44 \mathrm{~m}$ & $\mathrm{Se}^{73}$ & $\beta^{+} 1.7$ & & E 2.7 \\
\hline $45 \mathrm{~m}$ & $\operatorname{Mn} 51$ & $\beta+2.2$ & & E 3.2 \\
\hline
\end{tabular}

- Total Disintegration Energy (Mev), or Isotopic Mass

Page 20. 


\begin{tabular}{|c|c|c|c|c|}
\hline$\underline{B g} f-L i f e$ & Isotope & $\begin{array}{l}\text { Particles and } \\
\text { Energies (Mov) }\end{array}$ & $\begin{array}{c}\text { Gamma Enerzies } \\
\frac{(\mathrm{Mev})}{}\end{array}$ & $\begin{array}{l}\text { Total Energs } \\
\text { or Mass" }\end{array}$ \\
\hline $45 \mathrm{~m}$ & $\mathrm{Cs}^{125}$ & $\beta^{+} 2.05, \ldots$ & $\begin{array}{l}K \\
r .111\end{array}$ & \\
\hline $47 \mathrm{~m}$ & $\mathrm{Po}^{203}$ & & K & \\
\hline $47 \mathrm{~m}$ & $\mathrm{Bi}^{213}$ & $\begin{array}{l}\beta^{-} 1.39, .96 \\
\text { a } 5.9\end{array}$ & $r .43$ & \\
\hline $48 \mathrm{~m}$ & $\mathrm{Au}^{200}$ & $\beta^{-} 2.2$ & $r .39,1.13$ & E 2.2 \\
\hline $49 \mathrm{~m}$ & $\mathrm{Ta} 185$ & $\begin{array}{l}\beta^{-} 1.7 \\
e^{-}\end{array}$ & $\gamma .13, .18, .24$ & \\
\hline $50 \mathrm{~m}$ & $\mathrm{Sn}^{126}$ & $\beta^{-}$ & & \\
\hline $50 \mathrm{~m}$ & $\mathrm{Cd}^{117}$ & $\beta-1.6,3.0$ & $r 1.3, .27-2.0$ & \\
\hline $50 \mathrm{~m}$ & $\mathrm{~Np}^{231}$ & a 6.28 & & M 231.111 \\
\hline $50 \mathrm{~m}$ & As 70 & $\beta^{+} 1.4,2.5$ & $r 1.1,2.0, \ldots$ & E 6.6 \\
\hline $50 \mathrm{~m}$ & $\mathrm{Y}^{91}$ & $0^{-}$ & I.T. .55 & \\
\hline $50 \mathrm{~m}$ & In 108 & $\begin{array}{l}\beta^{+} 2.3 \\
e^{-}\end{array}$ & & \\
\hline $52 \mathrm{~m}$ & $\mathrm{I}^{134}$ & $\beta^{-}-1.5,2.5$ & $r .86,1.1,1.8$ & E 3.4 \\
\hline $52 \mathrm{~m}$ & $\mathrm{zn}^{69}$ & $\beta^{-} .90$ & & E. .90 \\
\hline $52 \mathrm{~m}$ & $\mathrm{Tc} 96$ & $\begin{array}{l}\beta^{+} \\
\theta^{-}\end{array}$ & I.T. .034 & \\
\hline $53 \mathrm{~m}$ & $\mathrm{Tc}^{94}$ & $\beta^{+} 2.41, \cdots$ & 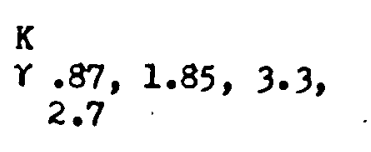 & E 4.30 \\
\hline $54 \mathrm{~m}$ & $\operatorname{Rh}^{103}$ & $e^{-}$ & I.T. ..$\alpha_{4} 0$ & \\
\hline $54.0 \mathrm{~m}$ & $\ln 116$ & $\beta^{-1.00, \cdots}$ & $\begin{array}{l}r 1.27,1.09,2.1, \\
1.5, \ldots\end{array}$ & B 3.36 \\
\hline $55 \mathrm{~m}$ & $c 139$ & $\beta-3.0,1.7$ & $r .3,1.3$ & E 3.3 \\
\hline $55 \mathrm{~m}$ & cd 105 & $\beta^{+} 1.69, \cdots$ & $\stackrel{k}{r} \cdot 025-2 \cdot 32$ & \\
\hline $56 \mathrm{~m}$ & $\mathrm{Po}^{202}$ & a 5.59 & $\mathrm{~K}$ & · \\
\hline
\end{tabular}

- Total Disintegration Energy (Mev), or Isotopic Mass

Page 21. 


\begin{tabular}{|c|c|c|c|c|}
\hline 嵓If-Life & Isotope & $\begin{array}{l}\text { Particles and } \\
\text { Energies (Mev) }\end{array}$ & $\begin{array}{c}\text { Gamma Energies } \\
(\mathrm{Mev})\end{array}$ & $\begin{array}{l}\text { Total Enerzo } \\
\text { or Mass }\end{array}$ \\
\hline $57 \mathrm{~m}$ & $S_{\theta}^{81}$ & $\theta^{-}$ & I.T. .103 & \\
\hline $57 \mathrm{~m}$ & $\mathrm{Hg}^{191}$ & $e^{-}$ & $\begin{array}{l}K \\
\gamma .253, \ldots\end{array}$ & \\
\hline $57 \mathrm{~m}$ & $S c 49$ & $\beta-2.0$ & no $r$ & $E 2.0$ \\
\hline $58 \mathrm{~m}$ & $u^{229} \cdots$ & a 6.42 & $-\mathbf{K}$ & M 229.105 \\
\hline $58 \mathrm{n}$ & {$\left[a^{131}\right.$} & $\beta+1.6$ & & \\
\hline $59 \mathrm{~m}$ & $\operatorname{col} \alpha_{4}$ & & $\begin{array}{l}\mathrm{K} \\
\mathrm{r} .067, .084, .124, \\
\end{array}$ & \\
\hline$<1 \mathrm{~h}$ & $R_{h} 109$ & . & & \\
\hline $1 \mathrm{~h}$ & $\mathrm{Sb}^{116}$ & $\beta^{+} 1.4$ & $\gamma 1.27, .9, .41$ & E 4.7 \\
\hline $2 \mathrm{~h}$ & $E u^{158}$ & $\beta-2.6$ & $r$ & - \\
\hline I h & $\mathrm{Ra}^{230}$ & $\beta^{-} 1.2$ & & \\
\hline $1 \mathrm{~h}$ & $\mathrm{Mv}^{256}$ & & K & \\
\hline I h & $\mathrm{Np}^{240}$ & $\beta^{-} .90$ & $\gamma .56, .43, .16-1.16$ & E 2.06 \\
\hline $1 \mathrm{~h}$ & $\mathrm{Ru}^{94}$ & $\mathrm{~K}$ & . & \\
\hline $1.0 \mathrm{~h}$ & $\mathrm{Bi}^{201}$ & a 5.15 & K & \\
\hline$\sim 1 \mathrm{~h}$ & $\mathrm{Am}^{237}$ & a 6.01 & K & M. 237.124 \\
\hline$\sim 1 \mathrm{~h}$ & $\mathrm{Sb}^{128}$ & $\beta^{-}$ & & \\
\hline $1.02 \mathrm{~h}$ & $\mathrm{Bi}^{212}$ & $\begin{array}{l}\beta-2.25 \\
\alpha 6.05,6.09, \cdots, \\
\quad(9.5-10.5)\end{array}$ & $r . \alpha_{4} a-2.20$ & E 2.25 \\
\hline $1.05 \mathrm{~h}$ & $\mathrm{Te}^{133}$ & $e^{-}$ & I.T..- .4 & \\
\hline $1.09 \mathrm{~h}$ & $A c^{229}$ & $\beta^{-}$ & & \\
\hline $1.09 \mathrm{~h}$ & $\operatorname{In} 110$ & $\beta^{+} 2.25$ & $\begin{array}{l}\mathrm{K} \\
r .66 \mathrm{D}\end{array}$ & E 3.93 \\
\hline $1.1 \mathrm{~h}$ & $\operatorname{In}^{117}$ & $\beta^{-} .74$ & $\gamma .56, .16$ & E 1.46 \\
\hline
\end{tabular}

- Total Disintegration Energy (Mev), or Isotopic Mass 


\begin{tabular}{|c|c|c|c|c|}
\hline 西lf-Life & Isotope & $\begin{array}{l}\text { Particles and } \\
\text { Energles (Mev) }\end{array}$ & $\begin{array}{l}\text { Gamna Energies } \\
\text { (Mev) }\end{array}$ & $\begin{array}{l}\text { Total Ene } \\
\text { or Mass } \\
\end{array}$ \\
\hline $1.1 \mathrm{~h}$ & $\mathrm{Ag}^{103}$ & $\beta^{+} 1.3$ & $\begin{array}{l}k \\
\gamma .76, .55\end{array}$ & \\
\hline $1.13 \mathrm{~h}$ & $\mathrm{~Pb}^{2 \alpha_{4}}$ & $e^{-}$ & $\begin{array}{l}\text { I.T. .91 } \\
r \cdot 37 \mathrm{D}, .89 \mathrm{D}, \cdots\end{array}$ & \\
\hline $1.13 \mathrm{~h}$ & $\mathrm{Ga}^{68}$ & $\beta^{+} 1.88, .78$ & $\begin{array}{l}K \\
r 1.10\end{array}$ & E 2.90 \\
\hline $1.17 \mathrm{~h}$ & $\operatorname{Pr} 136$ & $\beta^{+} 2.0$ & $r .17, .8,1.1$ & \\
\hline $1.17 \mathrm{~h}$ & $\mathrm{Sr}^{85}$ & . & 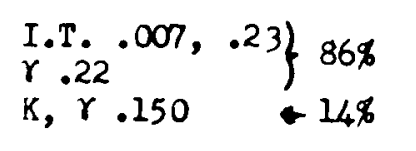 & \\
\hline $1.17 \mathrm{~h}$ & $\mathrm{Y}^{82}$ & $\beta^{+} 2$ & & \\
\hline $1.2 \mathrm{~h}$ & $\mathrm{Tl}^{195}$ & & & \\
\hline $1.2 \mathrm{~h}$ & $\mathrm{kr} \mathrm{r}^{77}$ & $\beta^{+} 1.86,1.67, \cdots$ & $\begin{array}{l}K \\
r .108, .131, .024, \\
.149-.87\end{array}$ & \\
\hline $1.20 \mathrm{~h}$ & $\mathrm{Te}^{129}$ & $\beta^{-1.46,1.01, \ldots}$ & $\gamma .03, .45,1.08, \cdots$ & E 1.49 \\
\hline $1.20 \mathrm{~h}$ & $\mathrm{NB}^{97}$ & $\beta^{-1.27}$ & $r .66$ & E 1.93 \\
\hline $1.23 \mathrm{~h}$ & $\mathrm{Yb}^{1653}$ & $\beta^{+} 2.4$ & & \\
\hline $1.25 \mathrm{~h}$ & $\operatorname{Er}^{163}$ & & $\stackrel{K}{r .43,1.10}$ & \\
\hline $1.28 \mathrm{~h}$ & $\mathrm{La}^{142}$ & $\beta^{-}>2.5$ & $\gamma .63, .87$ & \\
\hline $1.3 \mathrm{~h}$ & $w^{176}$ & $\begin{array}{l}\beta^{+} \sim 2 \\
e^{-}\end{array}$ & $\begin{array}{l}K \\
r \sim 1.3\end{array}$ & \\
\hline $1.30 \mathrm{~h}$ & $K r^{87}$ & $\beta^{-} 3.8 ; 1.3, \sim 3.3$ & $r .40,2.57, .85, \cdots$ & E 4.2 \\
\hline$>1.3 \mathrm{~h}$ & $I^{120}$ & $\beta^{+} 4.0$ & & \\
\hline $1.37 \mathrm{~h}$ & $\mathrm{Ge}^{75}$ & $\beta-1.18, .92, \cdots$ & $r .27, .07-.63$ & E 1.18 \\
\hline $1.4 \mathrm{~h}$ & $P t^{197}$ & $e^{-}$ & I.T. .34 & \\
\hline $1.4 \mathrm{~h}$ & $I^{121}$ & $\beta^{+} 1.13, \cdots$ & $r .213$ & \\
\hline $1.42 \mathrm{~h}$ & $\mathrm{Ba}^{139}$ & $\begin{array}{l}\beta^{-2}-22, .8,2.38 \\
\theta^{-}\end{array}$ & $\gamma .165 \mathrm{D}, 1.43$ & E 2.38 \\
\hline
\end{tabular}

* Total Disintegration Energy (Mev), or Isotopic Mass 


\begin{tabular}{|c|c|c|c|c|}
\hline Helf-Life & Isotope & $\begin{array}{l}\text { Particles and } \\
\text { Energies (Mev) }\end{array}$ & $\begin{array}{c}\text { Gamma Energies } \\
(\mathrm{Mev})\end{array}$ & $\begin{array}{l}\text { Total Ene } \\
\text { or Mass } \\
\end{array}$ \\
\hline $1.43 \mathrm{~h}$ & $\mathrm{Ge}^{78}$ & $\beta^{-} .9$ & $r$ & \\
\hline $1.5 \mathrm{~h}$ & $\mathrm{~Pb} 199$ & & $\begin{array}{l}K \\
\gamma .37, .35, .72\end{array}$ & \\
\hline $1.5 \mathrm{~h}$ & $P^{205}$ & a 5.2 & $K$ & \\
\hline $1.50 \mathrm{~h}$ & $\mathrm{Hg}^{190}$ & $e^{-}$ & K & \\
\hline $1.50 \mathrm{~h}$ & As $^{78}$ & $\beta^{-} 4.1, \cdots$ & $r$ & E 4.1 \\
\hline $1.6 \mathrm{~h}$ & $\mathrm{Ho}^{169}$ & $\beta^{-}$ & & \\
\hline $1.6 \mathrm{~h}$ & $\mathrm{Bi}^{20 R}$ & & K & \\
\hline $1.6 \mathrm{~h}$ & $\mathrm{Br}^{75}$ & $\beta^{+} 1.70, \cdots$ & $\begin{array}{l}K \\
r .6\end{array}$ & \\
\hline $1.6 \mathrm{~h}$ & $2 r^{87}$ & $\beta+2.10$ & $\stackrel{K}{r} \cdot 6, \cdot 3$ & E 3.50 \\
\hline $1.62 \mathrm{~h}$ & $\mathrm{Ba}^{126}$ & $\left(\beta^{+} 3.8\right)$ & $\begin{array}{l}\mathrm{K} \\
\gamma .22, .70,(.38)\end{array}$ & \\
\hline $1.63 \mathrm{~h}$ & $\operatorname{san} 95$ & $\beta^{+} 1.2$ & $\begin{array}{l}K \\
r \\
r\end{array}$ & E 2.2 \\
\hline $1.65 \mathrm{~h}$ & $\mathrm{Co}^{61}$ & $\beta^{-1.22}$ & $r .070$ & E 1.29 \\
\hline $1.7 \mathrm{~h}$ & $\Delta t^{208}$ & $\propto 5.65$ & $\begin{array}{l}K \\
\gamma .18, .66\end{array}$ & \\
\hline $1.73 \mathrm{~h}$ & $\operatorname{In}^{113}$ & $e^{-}$ & I.T. .39 & \\
\hline $1.8 \mathrm{~h}$ & $\mathrm{Nd} 149$ & $\beta-1.5, \cdots$ & $r .11, .03-.65$ & E 1.6 \\
\hline $1.8 \mathrm{~h}$ & $\mathrm{HF}^{170}$ & $\beta+2.4$ & & ' \\
\hline $1.8 \mathrm{~h}$ & At 207 & a 5.75 & $\mathrm{~K}$ & \\
\hline $1.8 \mathrm{~h}$ & $\mathrm{Xe}^{123}$ & $\beta^{+} 1.7$ & $\stackrel{K}{r} .15$ & \\
\hline $1.82 \mathrm{~h}$ & $A^{41}$ & $\beta^{-} 1.20,2.49$ & $r .129 D$ & E 2.49 \\
\hline $1.86 \mathrm{~h}$ & $k r^{83}$ & & $\underset{r . T .009}{.032}$ & \\
\hline
\end{tabular}

* Total Disintegration Energy (Mev), or Isotopic Mass 


\begin{tabular}{|c|c|c|c|c|}
\hline 峴f-Life & Isotope & $\begin{array}{l}\text { Particles and } \\
\text { Energies (Mev) }\end{array}$ & $\begin{array}{l}\text { Gemme Energies } \\
\frac{(\mathrm{Mev})}{}\end{array}$ & $\begin{array}{l}\text { Total Energy } \\
\text { or Mass* } \\
\end{array}$ \\
\hline $1.87 \mathrm{~h}$ & $F^{18}$ & $\beta^{+} .65$ & & E 1.67 \\
\hline $1.9 \mathrm{~h}$ & $\mathrm{Tl} 198$ & & $\begin{array}{l}K \\
I . T . \\
r .28, .049, .44-.64\end{array}$ & \\
\hline $1.9 \mathrm{~h}$ & $\mathrm{Am}^{238}$ & $e^{-}$ & $\begin{array}{l}K \\
\gamma .98, .58, .10 R\end{array}$ & \\
\hline $1.9 \mathrm{~h}$ & $\operatorname{In} 117$ & $\beta^{-} 1.77,1.61$ & $\underset{r . T .16}{\mathrm{r}} \cdot 31$ & \\
\hline $1.9 \mathrm{~h}$ & $\mathrm{Ba}^{129}$ & $\begin{array}{l}\beta^{+} 1.6 \\
e^{-} .13\end{array}$ & $r$ & \\
\hline $1.9 \mathrm{~h}$ & $\mathrm{Nb}^{89}$ & $\beta^{+} 2.9$ & & E 3.9 \\
\hline $2 \mathrm{~h}$ & $E^{249}$ & $\alpha 6.76$ & & M 249.154 \\
\hline $2 h$ & $\mathrm{Rh}^{106}$ & $\beta^{-} \sim 1$ & $\gamma .2-1.5$ & \\
\hline$\sim 2 \mathrm{~h}$ & $\mathrm{NB}^{89}$ & $\beta^{+}$ & $(Y .59)$ & \\
\hline$\sim 2 \mathrm{~h}$ & $B 1^{201}$ & . & $\mathbf{K}$ & \\
\hline $2.0 \mathrm{~h}$ & $\operatorname{Pr}^{138}$ & $\beta^{+} 1.4$ & $\gamma 1.0, .8, .3-1.7$ & \\
\hline $2.0 \mathrm{~h}$ & $A \pi^{2} 45$ & $\beta^{-} .90$ & $\begin{array}{l}r .036, .12, .26, \\
.06-.23\end{array}$ & \\
\hline $2.0 \mathrm{~h}$ & $\mathrm{Yb}^{177}$ & $\beta^{-1.30, \cdots}$ & $r_{\substack{1.23 \\
1.260}} .119,1.08$ & E 1.30 \\
\hline $2.1 \mathrm{~h}$ & $\mathrm{Ta}^{178}$ & $\begin{array}{l}\beta^{+} \sim 1 \\
\theta^{-} \sim .1\end{array}$ & $\begin{array}{l}K \\
\gamma \sim 1.4\end{array}$ & \\
\hline $2.2 \mathrm{~h}$ & $w^{177}$ & $e^{-} .13, \sim .4$ & $\begin{array}{l}K \\
r \sim .5,1.2\end{array}$ & \\
\hline $2.3 \mathrm{~h}$ & $\mathrm{I}^{132}$ & $\beta^{-} .9-2.12$ & $\gamma .67, .78, .53-2.2$ & E 3.57 \\
\hline $2.3 \mathrm{~h}$ & $D_{y} \leqq 253$ & a 3.6 & & \\
\hline $2.3 \mathrm{~h}$ & $\operatorname{Ir} 195$ & $\beta=1.2,2.1, \cdots$ & $r .42, .66, .88,>1$ & E 2.1 \\
\hline $2.3 \mathrm{~h}$ & $\mathrm{~Pb}^{198}$ & & $\begin{array}{l}K \\
r .17, .29, .36 \\
.28-.42\end{array}$ & \\
\hline
\end{tabular}

* Total Disintegration EnerEJ (Mev), or Isotopic Mass 


\begin{tabular}{|c|c|c|c|c|}
\hline Half-Lffe & Isotope & $\begin{array}{l}\text { Particles and } \\
\text { Energles (Mev) }\end{array}$ & $\begin{array}{l}\text { Gamma Energies } \\
\text { (Mev) } \\
\end{array}$ & $\begin{array}{l}\text { Totel Energy } \\
\text { or Mass }\end{array}$ \\
\hline $2.3 \mathrm{~h}$ & $\mathrm{Cm}^{238}$ & a 6.50 & K & M 238.128 \\
\hline $2.3 \mathrm{~h}$ & $B r^{83}$ & $\beta^{-} .94, \cdots$ & $r .051,(032, .009)$ & E .98 \\
\hline $2.32 \mathrm{~h}$ & $D_{y}^{165}$ & $\beta-1.25, \cdots$ & $r . \infty-1.0$ & E 1.25 \\
\hline $2.4 \mathrm{~h}$ & $\mathrm{Nd}^{1 / 4}$ & $\beta^{+} .7$ & $\begin{array}{l}K \\
Y 1.1\end{array}$ & E 1.7 \\
\hline $2.5 \mathrm{~h}$ & $\mathrm{Cr}^{247}$ & & $\begin{array}{l}K \\
\gamma .28, .49\end{array}$ & \\
\hline $2.5 \mathrm{~h}$ & Ho161 & & $\begin{array}{l}K \\
\gamma .090, .17\end{array}$ & \\
\hline $2.5 \mathrm{~h}$ & $\mathrm{Te}^{117}$ & $\beta^{+} 2.5$ & & \\
\hline $2.5 \mathrm{~h}$ & $c_{\theta}^{66}$ & $\beta^{+}(3)$ & K & \\
\hline $2.56 \mathrm{~h}$ & $\mathrm{Ni}^{65}$ & $\beta^{-2.10, .6,1.0}$ & $r 1.5,1.12, .37$ & E 2.10 \\
\hline $2.58 \mathrm{~h}$ & $\mathrm{Mn}^{56}$ & $\beta^{-} 2.8,1.0, .7$ & $r .85,1.8,2.1-3$ & E 3.7 \\
\hline $2.6 \mathrm{~h}$ & $A t^{206}$ & & $\mathrm{~K}$ & \\
\hline $2.62 \mathrm{~h}$ & $\mathrm{Si}^{31}$ & $\beta^{-1.48, \cdots}$ & $r 1.26$ & E 1.48 \\
\hline $2.7 \mathrm{~h}$ & $\operatorname{En}^{210}$ & a $6 . \alpha$ & K & M 210.057 \\
\hline $2.7 \mathrm{~h}$ & $\operatorname{Pm}^{150}$ & $\theta^{-2.0,3.0}$ & $\begin{array}{c}.34, .82, .43, \\
1.0-3.0\end{array}$ & - \\
\hline $2.7 \mathrm{~h}$ & $T^{93}$ & $\beta^{+} .80, .6$ & $\begin{array}{l}K \\
\gamma \\
1.3,1.5,2.0\end{array}$ & E 3.1 \\
\hline $2.7 \mathrm{~h}$ & $\mathrm{Sr}^{92}$ & $\beta^{-} \sim 5.5$ & $r 1.38$ & E 1.9 \\
\hline $2.8 \mathrm{~h}$ & $k r^{88}$ & $\beta^{-} .52,2.7, \cdots$ & $\begin{array}{l}r 2.4, .19, .85, \\
.008-2.2\end{array}$ & E 2.9 \\
\hline $2.8 \mathrm{~h}$ & $\mathrm{Sb}^{117}$ & $\theta^{-}$ & 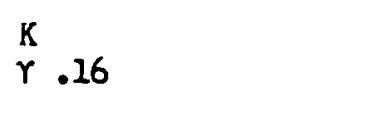 & \\
\hline $2.8 \mathrm{~h}$ & $\mathrm{TI} 197$ & & $\begin{array}{l}K \\
\gamma .152, .43, \cdots\end{array}$ & \\
\hline $2.8 \mathrm{~h}$ & $5 r^{87}$ & & I.T. .39 & \\
\hline
\end{tabular}

* Total Disintegration Energy (Mev), or Isotopic Mass 


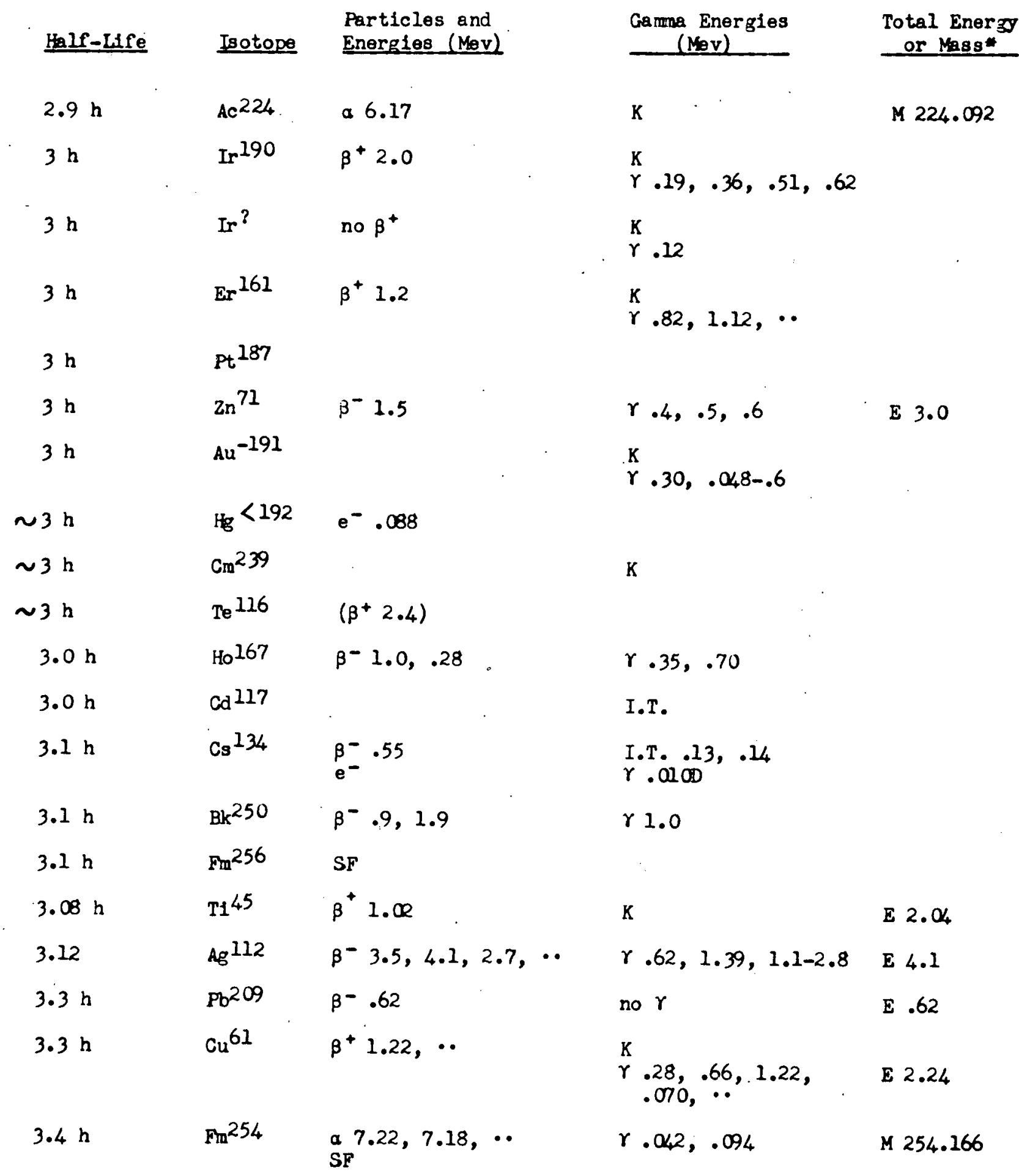

- Total Disintegration Energy (Mev), or Isotopic

Page 27. 


\begin{tabular}{|c|c|c|c|c|}
\hline 㫮lf-Life & Isotope & $\begin{array}{l}\text { Particles and } \\
\text { Energies (Mev) }\end{array}$ & $\begin{array}{l}\text { Gamma Energies } \\
\text { (Mev) }\end{array}$ & $\begin{array}{l}\text { Total En } \\
\text { or Mes } \\
\end{array}$ \\
\hline $3.5 \mathrm{~h}$ & $\mathrm{~Pb}^{20 R}$ & & $\begin{array}{l}\mathrm{I} . \mathrm{T} \cdot .79, .13 \\
\mathrm{~K} .96, .42, .39-.66\end{array}$ & \\
\hline $3.5 \mathrm{~h}$ & $Y^{92}$ & $\beta^{-} 3.60,2.7,1.3$ & $\gamma .94, .21-2.4$ & E 3.60 \\
\hline $3.5 \mathrm{~h}$ & $y^{83}$ & & & \\
\hline $3.7 \mathrm{~h}$ & $\mathrm{Lu} 176$ & $\beta^{-}-1.2, \cdots$ & $r .089$ & E 1.2 \\
\hline $3.7 \mathrm{~h}$ & $\mathrm{Y}^{84}$ & $\beta+2.0$ & $\begin{array}{l}K \\
\gamma\end{array}$ & \\
\hline $3.8 \mathrm{~h}$ & $\mathrm{PO}^{204}$ & a 5.37 & $\mathrm{~K}$ & \\
\hline $3.8 \mathrm{~h}$ & $2 a^{1 / 1}$ & $\beta^{-2.43, .9}$ & $r \sim 1.5$ & E 2.43 \\
\hline $3.9 \mathrm{~h}$ & $\mathrm{Sc}^{43}$ & $\beta^{+} 1.19, .82, .39$ & $r .37, .62, .25, .84$ & E 2.21 \\
\hline $4 h$ & Lal33 & $\begin{array}{l}\beta^{+} 1.2 \\
e^{-} .26\end{array}$ & $\begin{array}{l}K \\
r .8\end{array}$ & \\
\hline $4 h$ & $\mathrm{Sn}^{108}$ & & K & \\
\hline $4 \mathrm{~h}$ & $\mathrm{Sn}^{110}$ & ; & $\stackrel{K}{r} .28$ & \\
\hline$\sim 4 \mathrm{~h}$ & $\mathrm{Tr}^{196}$ & & $\begin{array}{l}\mathrm{K} \\
\gamma \cdot 43\end{array}$ & \\
\hline $4.0 \mathrm{~h}$ & $\mathrm{Lu}^{172}$ & $\beta+1.2$ & & \\
\hline $4.0 \mathrm{~h}$ & $\mathrm{Sc} 44$ & $\beta^{+} 1.47$ & $\begin{array}{l}K \\
\gamma \\
1.16,2.54\end{array}$ & E 3.65 \\
\hline $4.1 \mathrm{~h}$ & $\mathrm{~Tb}^{149}$ & a 3.95 & $K(?)$ & \\
\hline $4.3 \mathrm{~h}$ & $\operatorname{In}^{109}$ & $\beta^{+} .7$ & $\begin{array}{l}K \\
\gamma .058, .20, .35, .43\end{array}$ & \\
\hline $4.4 \mathrm{~h}$ & $\mathrm{Bk}^{244}$ & a 6.66 & $\begin{array}{l}K \\
\gamma .90-1.72\end{array}$ & \\
\hline $4.4 \mathrm{~h}$ & $\mathrm{kr} \mathrm{r}^{85}$ & $\beta^{-} .83$ & $\mathrm{I}_{\mathrm{r} . \mathrm{T}}^{\mathrm{T} .} .31$ & \\
\hline $4.5 \mathrm{~h}$ & $? \mathrm{Rh}^{99}$ & $\beta^{+} .74$ & $\gamma .29$ & \\
\hline
\end{tabular}

* Total Disintegration Energy (Mev), or. Isotopic Mass 


\begin{tabular}{|c|c|c|c|c|}
\hline Half-Life & Isotope & $\begin{array}{l}\text { Particles and } \\
\text { Energies (Mev) }\end{array}$ & $\begin{array}{c}\text { Gamma Energies } \\
(\mathrm{MeV})\end{array}$ & $\begin{array}{l}\text { Total Energy } \\
\text { or Mass* }\end{array}$ \\
\hline $4.5 \mathrm{~h}$ & $\mathrm{Bk}^{243}$ & $a 6.55,6.72,6.20$ & $\begin{array}{l}K \\
\gamma .74, .84, .96, . .\end{array}$ & M 243.139 \\
\hline $4.5 \mathrm{~h}$ & $\operatorname{Pr} 139$ & $\beta^{+} 1.0$ & $r 1.3,1.6, .17 ?$ & \\
\hline $4.5 \mathrm{~h}$ & $\mathrm{La}^{132}$ & $\beta+3.5$ & $\gamma 1.0$ & \\
\hline $4.5 \mathrm{~h}$ & $\operatorname{In}^{115}$ & $\begin{array}{l}\beta^{-} .83 \\
\mathrm{e}^{-}\end{array}$ & I.T. . 33 & \\
\hline $4.5 \mathrm{~h}$ & $\operatorname{Ban} 105$ & $\beta^{-1.15}$ & $\gamma .73,(.130)$ & $E 2.08$ \\
\hline $4.6 \mathrm{~h}$ & $\mathrm{Br} \mathrm{r}^{80}$ & $e^{-}$ & $\mathrm{I}_{\mathrm{r} . \mathrm{T} . \mathrm{\alpha}_{4}}^{.05}$ & \\
\hline $4.6 \mathrm{~h}$ & $\mathrm{Sb}^{129}$ & $\beta^{-} .92,1.70$ & $\gamma .16-.78$ & \\
\hline $4.7 \mathrm{~h}$ & $\mathrm{Rb}^{81}$ & $\beta+1.0$ & $\mathrm{~K} .9,(.19)$ & E 2.2 \\
\hline $5 \mathrm{~h}$ & $\mathrm{Y}^{85}$ & & & \\
\hline $5 \mathrm{~h}$ & $\mathrm{Hg}^{193}$ & & $\begin{array}{l}K \\
\gamma .038, .032-1.63\end{array}$ & \\
\hline $5 \mathrm{~h}$ & $\mathrm{To}^{156}$ & $\beta^{-} \cdot 14$ & & \\
\hline $5 \mathrm{~h}$ & $\mathrm{Ga}^{73}$ & $\beta-1.4$ & $(r .054, .0230)$ & E 1.5 \\
\hline $5 \mathrm{~h}$ & $\mathrm{TI}^{198}$ & & $\begin{array}{l}K \\
\gamma .410, .68, .19-1.20\end{array}$ & \\
\hline$\sim 5 \mathrm{~h}$ & $2 u^{179}$ & $\beta^{-}$ & & \\
\hline $5.0 \mathrm{~h}$ & Ho 160 & & ${ }_{X}^{\gamma} .19, .72, .96, \cdots$ & \\
\hline $5.0 \mathrm{~h}$ & $\mathrm{Pu}^{243}$ & $\mathrm{\beta}^{-} \cdot 57, .48, \cdots$ & $r .085, \cdots$ & E. .57 \\
\hline $5.0 \mathrm{~h}$ & $\operatorname{In} 110$ & & $\begin{array}{l}\mathrm{K} \\
\mathrm{I} . \mathrm{T} . \\
\mathrm{r} .66, .12 \\
.88, .94\end{array}$ & \\
\hline $5.1 \mathrm{~h}$ & $\mathrm{Sb}^{118}$ & $\begin{array}{l}\beta^{+} .7 \\
e^{-} .2\end{array}$ & $\begin{array}{l}K \\
r .26,1.5\end{array}$ & \\
\hline
\end{tabular}

* Total Disintegration Energy (Mev), or Isotopic Mass

Page 29. 


\begin{tabular}{|c|c|c|c|c|}
\hline Helf-Iffe & Isotope & $\begin{array}{l}\text { Particles and } \\
\text { Energles (Mev) }\end{array}$ & $\begin{array}{c}\text { Gamma Energies } \\
(\mathrm{Mev})\end{array}$ & $\begin{array}{l}\text { Total Ener } 50 \\
\text { or Mass }\end{array}$ \\
\hline $5.3 \mathrm{~h}$ & $\mathrm{Ag}^{113}$ & $\beta^{-} 2.0$ & $r .29$ & E 2.0 \\
\hline $5.5 \mathrm{~h}$ & $\mathrm{Na} 139$ & $\begin{array}{l}\beta^{+} 3.1 \\
e^{-} .28\end{array}$ & $\begin{array}{l}K \\
\gamma 1.3\end{array}$ & E 4.1 \\
\hline $5.5 \mathrm{~h}$ & $A t^{209}$ & a 5.64 & $\begin{array}{l}\mathrm{K} \\
\mathrm{\gamma} .195, .091, .55, .78\end{array}$ & \\
\hline $5.5 \mathrm{~h}$ & $\mathrm{Hf}^{180}$ & & $\begin{array}{l}\mathrm{I} \cdot \mathrm{T} . \\
r .057-.44\end{array}$ & \\
\hline $5.5 \mathrm{~h}$ & $\mathrm{Pd}^{\mathrm{III}}$ & $\beta^{-}$ & ${ }_{r}^{I . T} .16,1.77$ & \\
\hline $5.7 \mathrm{~h}$ & 80207 & a 5.10 & $\begin{array}{l}K \\
r .41, .74, .99, . .\end{array}$ & $M 207 . \alpha_{4} 7$ \\
\hline $5.7 \mathrm{~h}$ & Mo 90 & $\begin{array}{l}\beta^{+} \\
e^{-}\end{array}$ & $\underset{\mathrm{K}}{\mathrm{r}} .25 \mathrm{D},(.12)$ & \\
\hline $5.9 \mathrm{~h}$ & $\operatorname{Pr} 145$ & $\beta^{-1.7}$ & $r .070$ & \\
\hline $6 \mathrm{~h}$ & 0,190 & & I.T. ? & \\
\hline $6 \mathrm{~h}$ & $A t^{208}$ & & $\mathrm{~K}$ & \\
\hline $6 \mathrm{~h}$ & $\mathrm{Hg}^{192}$ & $\beta^{+} 1.2$ & 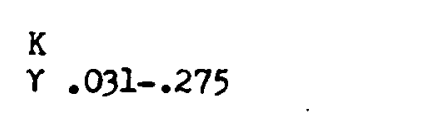 & . \\
\hline $6.0 \mathrm{~h}$ & $\mathrm{Tc}^{99}$ & $e^{-}$ & $\begin{array}{l}\text { I.T. . .00 , } .142 \\
r .140\end{array}$ & \\
\hline $6.13 \mathrm{~h}$ & $A c^{228}$ & $\beta^{-1.11, .45-2.18}$ & $\begin{array}{l}r .058, .10, .91 \\
.08-1.64\end{array}$ & E 2.18 \\
\hline $6.2 \mathrm{~h}$ & $\mathrm{Cs}^{127}$ & $\beta^{+} 1.06, .7, \cdots$ & $\begin{array}{l}K \\
\gamma .41, .125, .175-.44\end{array}$ & \\
\hline $6.3 \mathrm{~h}$ & $\mathrm{Ce}^{133}$ & $\beta+1.3$ & $\begin{array}{l}K \\
Y \\
1.8\end{array}$ & \\
\hline $6.3 \mathrm{~h}$ & $R b^{82}$ & $\beta^{+} .77, \cdots$ & 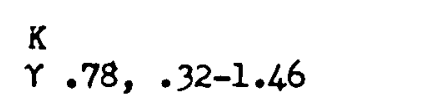 & \\
\hline $6.66 \mathrm{~h}$ & $\mathrm{~Pa}^{23 / 4}$ & $\beta^{-} .5, \cdots$ & $r . \alpha_{4}-1.7$ & M 234.117 \\
\hline $6.7 \mathrm{~h}$ & $\mathrm{I}^{135}$ & $\beta-1.0, .5,1.4$ & $r \frac{1.3,1.8,2.4}{(.52)}$ & \\
\hline
\end{tabular}

* Total Disintegration Energy (Mev), or Isotopic Mass 


\begin{tabular}{|c|c|c|c|c|}
\hline Half-Life & Isotope & $\begin{array}{l}\text { Perticles and } \\
\text { Energies (Mev) }\end{array}$ & $\frac{\text { Gamme Energies }}{(\mathrm{Mev})}$ & $\begin{array}{l}\text { Total Energs } \\
\text { or Mass }\end{array}$ \\
\hline $6.7 \mathrm{~h}$ & $\operatorname{col} 207$ & $\frac{\beta^{+}}{\left(e^{-}\right)} \cdot 32$ & $\stackrel{K}{\gamma .85,(.094)}$ & E 1.43 \\
\hline $6.9 \mathrm{~h}$ & Mo93 & $e^{-}$ & $\begin{array}{l}\text { I.T. } .26 \\
\gamma 1.48, .69\end{array}$ & \\
\hline $7 \mathrm{~h}$ & $\mathrm{~Tb}^{154}$ & $\beta^{-} ?$ & K & \\
\hline $7.1 \mathrm{~h}$ & $\mathrm{Se}^{73}$ & $\beta^{+} 1.29,1.65$ & $r .36 \mathrm{D}, .066 \mathrm{D}$ & E 2.74 \\
\hline $7.4 \mathrm{~h}$ & $\mathrm{TI}^{199}$ & & $\begin{array}{l}K \\
\gamma .33, .158, .208, \\
.247, .050-.49\end{array}$ & . \\
\hline $7.5 \mathrm{~h}$ & At 211 & a $5.86,(7.43, \ldots)$ & $\begin{array}{l}K, I \\
r .67,(.89, .57)\end{array}$ & $M 211.053$ \\
\hline $7.5 \mathrm{~h}$ & $\operatorname{Er}^{171}$ & $\beta^{-1.06, .67,1.48}$ & $r .113 D, .31, .12-.81$ & E 1.48 \\
\hline $7.7 \mathrm{~h}$ & $\operatorname{Tn} 166$ & $\begin{array}{l}\beta^{+} \\
\theta^{-}\end{array}$ & $\begin{array}{l}\mathrm{K} \\
\mathrm{Y} .08 \mathrm{D}-1.32\end{array}$ & \\
\hline $8 \mathrm{~h}$ & $P d 101$ & $\beta^{+} \cdot .5,2.37$ & $\mathrm{~K}$ & E $1.5(3)$ \\
\hline $8 \mathrm{~h}$ & $\mathrm{Fe}^{52}$ & $\beta^{+} .80,(2.7)$ & $\begin{array}{l}K \\
(\gamma \\
1.4, .39)\end{array}$ & E 2.21 \\
\hline $8.0 \mathrm{~h}$ & $\mathrm{Ta}^{176}$ & $\mathrm{e}^{-} \cdot 1, .2, \sim 1$ & $\begin{array}{l}K \\
r \sim 2\end{array}$ & \\
\hline $8.1 \mathrm{~h}$ & $\mathrm{Ta}^{180}$ & $\beta^{-} .70, \cdots$ & $\begin{array}{l}K \\
\gamma .093 D, .102\end{array}$ & \\
\hline $8.2 \mathrm{~h}$ & Dy 157 & & $\begin{array}{l}K \\
r \cdot 32\end{array}$ & \\
\hline $8.3 \mathrm{~h}$ & $A t^{210}$ & a $5.35-5.52$ & $\underset{2.6}{K} \underset{2.18, .25, .047-}{ }$ & M 210.053 \\
\hline $8.7 \mathrm{~h}$ & $\mathrm{Ta}^{184}$ & $\beta^{-1.26, .15}$ & $\begin{array}{r}r .40, .89, .24 \\
i .18, .11-.78\end{array}$ & \\
\hline $9 \mathrm{~h}$ & $\mathrm{Pu}^{234}$ & a 6.19 & K & M 234.117 \\
\hline $9 \mathrm{~h}$ & $\mathrm{Co}_{\theta} 137$ & . & $\begin{array}{l}K \\
r .44\end{array}$ & 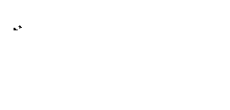 \\
\hline
\end{tabular}

- Total Disintegration Energy (Mev), or Isotoplc Mass 


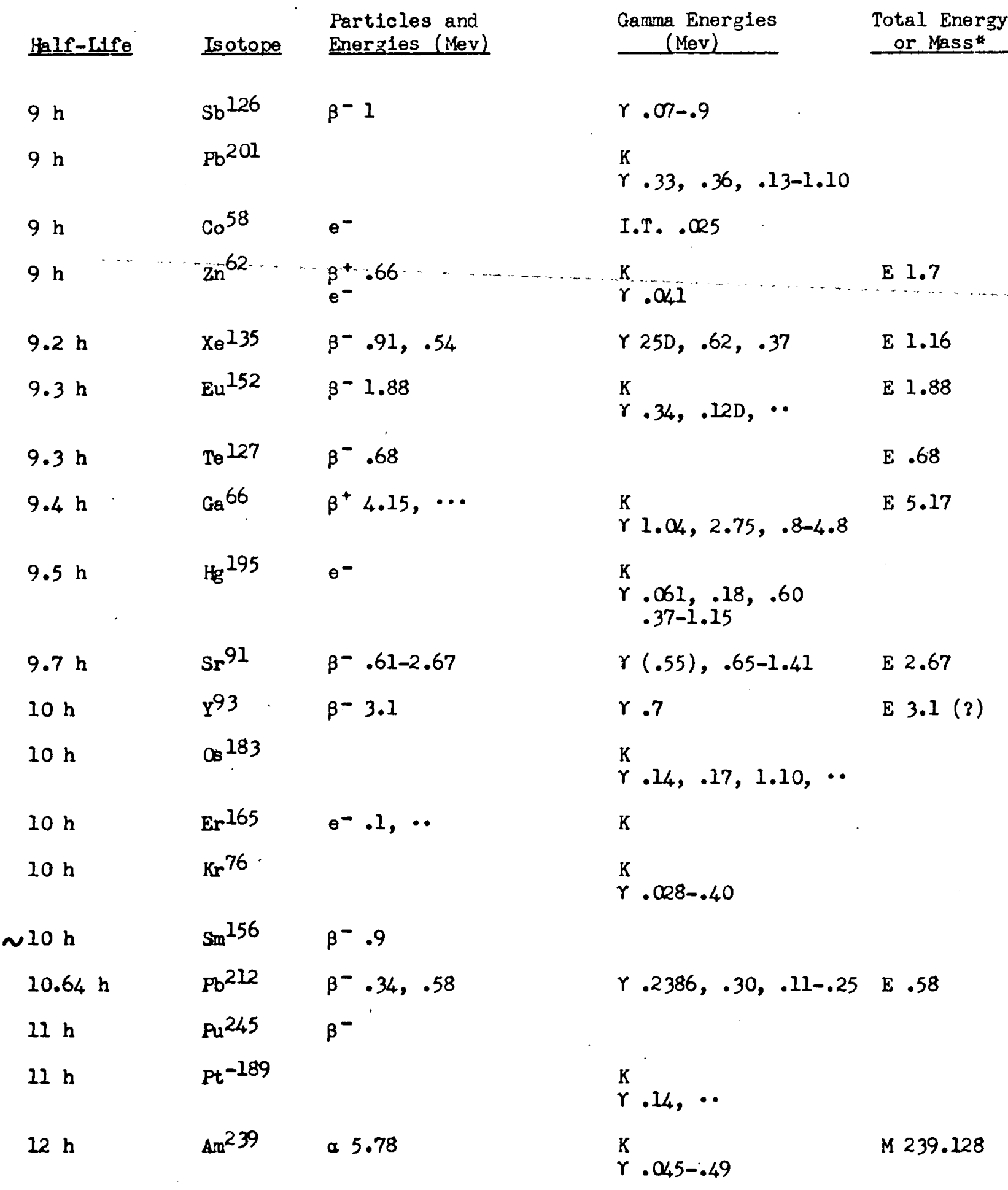

* Total Disintegration Energy (Mev), or Isotopic Mass 
$12 \mathrm{~h}$

$\mathrm{Hg}^{193}$

$12 \mathrm{~h}$

$12 \mathrm{~h}$

$12 \mathrm{~h}$

$12 \mathrm{~h}$

$12.5 \mathrm{~h}$

$12.6 \mathrm{~h}$

$12.8 \mathrm{~h}$

$13 \mathrm{~h}$

$13 \mathrm{~h}$

$13 \mathrm{~h}$

$13.6 \mathrm{~h}$

$14 \mathrm{~h}$

$14 \mathrm{~h}$

$14 \mathrm{~h}$

$u_{h} \mathrm{~h}$

$14 \mathrm{~h}$

$14.1 \mathrm{~h}$

$14.6 \mathrm{~h}$

$\mathrm{Bi}^{203}$

$\mathrm{Bi}^{2 \alpha_{4}}$

$\mathrm{Ge}^{77}$

$K^{42}$

$I^{130}$

$\mathrm{Nb}^{92}$

$R_{\theta}{ }^{182}$

$I^{123}$

Pd 109

$Y^{87}$

$\mathrm{Eu}^{150}$

$\alpha^{191}$

$\mathrm{U}^{240}$

$2 n^{69}$

$\mathrm{Ga}^{72}$

$\mathrm{Nb} 90$ a 4.85

$e^{-}$

$\mathrm{Ir}^{187} \quad \mathrm{\beta}^{+2.2}$

$\beta^{-2.20, \cdots}$

$\beta=3.5,2.0, \cdots$

$\beta^{-1 . \infty, .60}$

$\beta^{-} .57$

$\beta^{+} .66$

$\theta^{-}$

$\beta=1.0$

$\theta^{-}$

$\beta^{-1.1}$

$\beta^{-} .36,(2.16, \cdots)$

$\beta^{-} .64-3.17$
I.T. .101

$r .039$

$\mathrm{K}$

$\mathrm{K}$

$r .03-1.6$

K

$r .22, .08-1.23$

( $r .91, .37, .89)$

K

$r .30, .14, .44, \cdots$

$\gamma .21-2 \cdot 3$

E 2.7

$r 1.5, .32$

E 3.5

$r .74, .66, .53, .41$

E 2.95

$\mathrm{K}$

$\gamma 1.34$

$\mathrm{E}^{-} .57$

$\mathrm{K}$

$\gamma 2.35$

$\mathrm{K}$

$\gamma \cdot 11-.35, \sim 1$

$\mathrm{K}$

$\gamma .159$

(r.088)

E 1.1

I.T. .38

I.T. .074

$(\gamma .56, \cdots)$

E. .36

I.T. .44

$r .64, .60-3.35$,

E 4.00

$\gamma 1.14,2.23, .14, \cdots$

3.80

* Total Disintegration Energy (Mev), or Isotopic Mass 


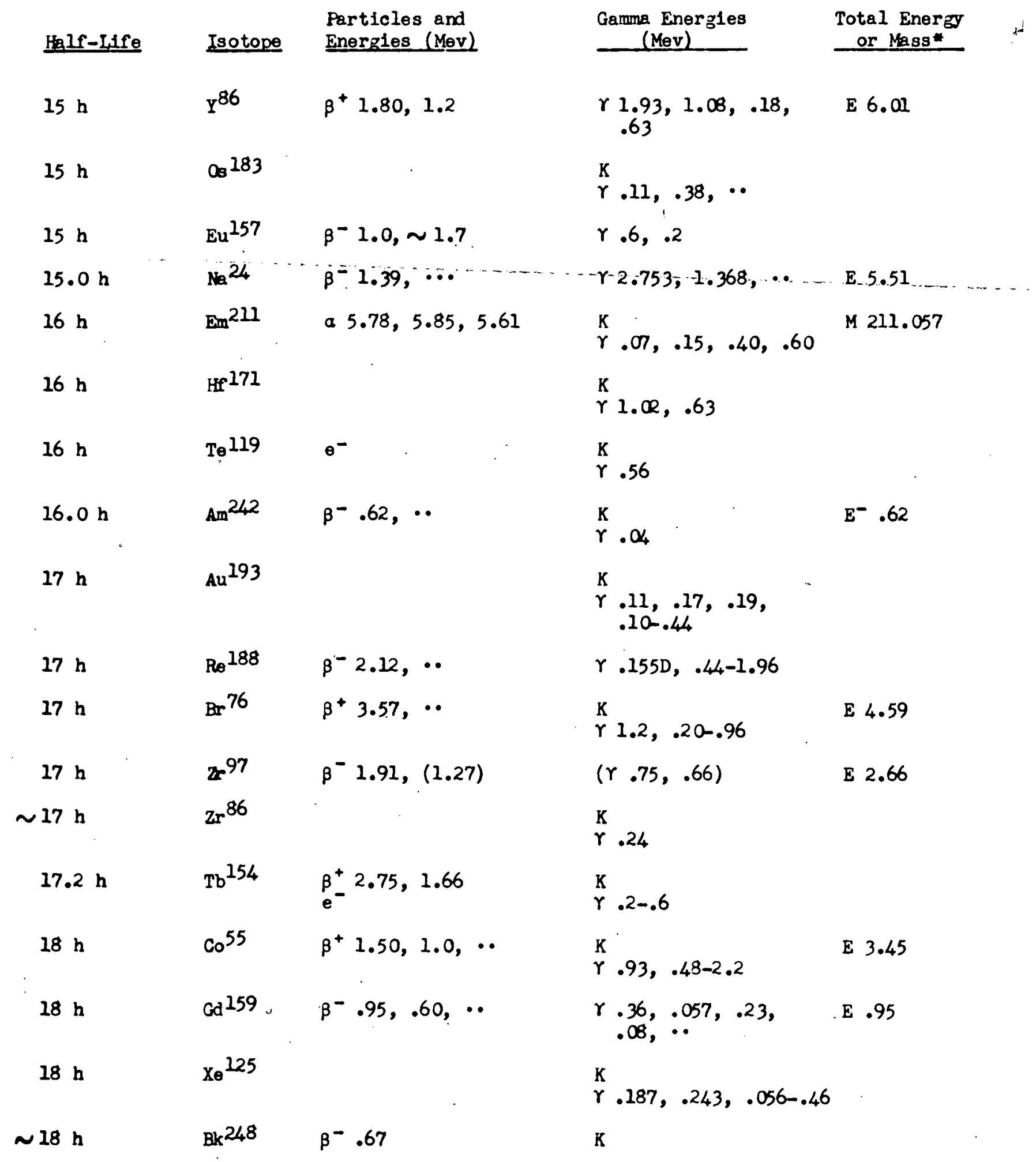

- Total Disintegration Energy (Mev), or Isotopic Mass 


\begin{tabular}{|c|c|c|c|c|}
\hline$\underline{H a} I f-L f f \theta$ & Isotope & $\begin{array}{l}\text { Particles and } \\
\text { Energies (Mev) }\end{array}$ & $\begin{array}{l}\text { Gamme Energies } \\
\text { (Mev) }\end{array}$ & $\begin{array}{l}\text { Total Energy } \\
\text { or Mass* } \\
\end{array}$ \\
\hline $19 \mathrm{~h}$ & Pt $^{197}$ & $\beta^{-} .67, .48, .47$ & $r .0770, .19, .28$ & E. .75 \\
\hline $19 h$ & $\operatorname{Ir}^{194}$ & $\beta-2.24,1.91, \cdots$ & $r .33, .64, .29-2.0$ & E 2.24 \\
\hline $19 \mathrm{~h}$ & $L_{0} 135$ & & $\begin{array}{l}\mathrm{K} \\
\mathrm{r} .49, .66\end{array}$ & \\
\hline $19 \mathrm{~h}$ & $\mathrm{~Tb}^{150}$ & a 3.4 & $K(?)$ & \\
\hline $19 \mathrm{~h}$ & $x_{\theta}^{122}$ & $\left(\beta^{+} 3.12\right)$ & $\frac{K}{r} .18, .23$ & \\
\hline $19.1 \mathrm{~h}$ & $\operatorname{Pr}^{142}$ & $\beta-2.16, .6$ & $r 1.59$ & B 2.16 \\
\hline $20 \mathrm{~h}$ & $\mathrm{Fm}^{255}$ & $\alpha 7.08$ & & . \\
\hline $20 \mathrm{~h}$ & $\mathrm{Tc}^{95}$ & & $\begin{array}{l}K \\
Y .76,1.1\end{array}$ & E 1.6 \\
\hline $21 \mathrm{~h}$ & $\mathrm{I}^{133}$ & $\beta^{-1.3, .4}$ & $\gamma .53, .85,1.4$ & E 1.8 \\
\hline $22 \mathrm{~h}$ & $\mathrm{~Pb}^{200}$ & & $\begin{array}{l}K \\
\gamma .148, .142, .24, . \\
.033-.45\end{array}$ & .27 , \\
\hline $21 \mathrm{~h}$ & $\operatorname{Rh}^{100}$ & $\beta+2.62, \cdots$ & $\begin{array}{l}K \\
\gamma .53, .44, .30-2.4\end{array}$ & E 3.64 \\
\hline $21 \mathrm{~h}$ & $\mathrm{Pd}^{112}$ & $\beta^{-} .28$ & $r .018$ & $E \cdot 30$ \\
\hline $21.3 \mathrm{~h}$ & $\mathrm{Mg}^{28}$ & $\beta^{-} .45$ & $r .032,1.35, .95$ & E 1.83 \\
\hline $22 \mathrm{~h}$ & $\mathrm{~Pa} 228$ & a $6.09,5.85$ & $\stackrel{K}{r .058, .130}$ & M 228.10 \\
\hline $22 \mathrm{~h}$ & $\mathrm{~Np}^{236}$ & $\beta^{-} .52, \cdots$ & $\mathrm{K} . \alpha_{4}, . \alpha_{44}$ & $\mathrm{~B}^{-} .52$ \\
\hline $22 \mathrm{~h}$ & $\mathrm{Ce}^{135}$ & $\beta^{+} .8$ & $\mathbf{K}$ & \\
\hline $22 \mathrm{~h}$ & $K^{-43}$ & $\beta^{-} .83, .24-1.84$ & $r .62, .37, \cdots$ & E 1.84 \\
\hline $23 \mathrm{~h}$ & $\mathrm{Cr}^{48}$ & $e^{-}$ & $\frac{K}{r} \cdot 30, .12$ & \\
\hline $23 \mathrm{~h}$ & $\mathrm{Nb} 96$ & $\beta^{-} .7, .4$ & $r .77, .22-1.19$ & E 3.1 \\
\hline
\end{tabular}

* Total Disintegration Enerzy (Mev), or Isotoplc Mass 
Helf-Lfe Isotope $\begin{aligned} & \text { Particles and } \\ & \text { Energies (Mev) }\end{aligned}$

$1 \mathrm{~d}$

$1 d$

ld

1. $\alpha_{4} d$

1. $\alpha_{4} \mathrm{~d}$

$1.07 \mathrm{~d}$

$1.08 \mathrm{~d}$

$1.12 \mathrm{~d}$

$1.13 \mathrm{~d}$

$1.13 \mathrm{~d}$

$1.13 \mathrm{~d}$

$1.13 \mathrm{~d}$

$1.21 \mathrm{~d}$

$1.21 \mathrm{~d}$

$1.21 \mathrm{~d}$

$1.25 \mathrm{~d}$

$1.25 \mathrm{~d}$

$1.29 \mathrm{~d}$

$1.29 \mathrm{~d}$ $\cos 182$

$\mathrm{w}^{187}$

HF 173

$\mathrm{Hg}^{197}$

$\operatorname{Tn}^{165}$

$\mathrm{Th}^{231}$

$\beta^{-} .09, .30, .22$

As 72

As 76

Ho 166

Pm 151

$\mathrm{Tl}^{200}$

$\beta-1.1$

$\beta^{+}$

$\beta+2.50,3.34, \cdots$

$\beta-2.96,2.41, \cdots$

$\beta^{-1.85, \cdots}$

$\mathrm{Sn}^{121} \quad \beta^{-} \cdot 38$

$\mathrm{Ba} 135 \quad \mathrm{e}^{-}$

$\mathrm{Ac}^{226} \quad \beta^{-} 1.2$

$\mathrm{Fm}^{252}$

a 7.1 .

Tel31

$\beta^{-} \cdot 4-2.5$

os 193

$\beta^{-1.10, \cdots}$

Cs 129
Er 160

\section{Gamma Energies \\ (Mev)}

Total Energy

or Mass*

K

$\gamma .072-.87$

E 1.31

K

$r .12, .30$

I.T. .165

$\mathrm{K}$

$\gamma .134 \mathrm{D},(.28, .13)$

$\mathrm{K}$

$\gamma .20, .81,1.16,1.38$

r.022D, .085D, .059-E.32 .23

$\mathrm{K}$

$\gamma .84, .690,1-3$

E 4.36

$r .55,1.19, .64$,

E 2.96 $1.4,2.1$

E 1.85

r.081D, 1.4-1.6

$r .34, .17, .06-1.5$

$\mathrm{K}$ $\gamma .37,1.20, .83, .58$,
$.116-1.52$

E.38

I.T. .268

E 1.2

K

M 252.162

I.T. .18

$\gamma .77, .05-1.2$

$\gamma .073 D, .14-.56 \quad$ E 1.10

K

$\gamma .38, .56$

* Total Disintegration Energy (Mev), or Isotopic Mass

Page 36. 


\begin{tabular}{|c|c|c|c|c|}
\hline Half-Lffe & Isotope & $\begin{array}{l}\text { Particles and } \\
\text { Energles (Mev) }\end{array}$ & $\begin{array}{l}\text { Gemme Energies } \\
\text { (Mev) }\end{array}$ & $\begin{array}{l}\text { Total Energy } \\
\text { or Mass } \\
\end{array}$ \\
\hline $1.31 \mathrm{~d}$ & $\mathrm{~Pa}^{232}$ & $\beta^{-} .28, .4-1.24$ & $r .047-1.15$ & E 1.24 \\
\hline $1.37 \mathrm{~d}$ & $\mathrm{Ce}^{1 / 43}$ & $\beta^{-} 1.09, .3-1.38$ & $r .29, . \infty-1.10$ & E 1.44 \\
\hline $1.37 \mathrm{~d}$ & $\mathrm{Sr}^{83}$ & $\begin{array}{l}\beta^{+} \\
\theta^{-}\end{array}$ & $\begin{array}{l}K \\
r .04-.16\end{array}$ & \\
\hline $1.42 \mathrm{~d}$ & $K \mathbf{r}^{79}$ & $\beta^{+} .60, .34$ & $\begin{array}{l}K, L \\
r .26, .044, .08-.83\end{array}$ & E 1.62 \\
\hline $1.46 \mathrm{~d}$ & $\mathrm{C}_{\theta} 137$ & $e^{-}$ & I.T. . .26 & \\
\hline $1.46 \mathrm{~d}$ & $\alpha_{s}^{187}$ & & I.T. ? & \\
\hline $2.5 d$ & $\mathrm{~Pa}^{229}$ & a 5.69 & $\mathrm{~K}$ & M 229.104 \\
\hline $1.5 \mathrm{~d}$ & $E^{251}$ & a 6.48 & $K$ & M 251.159 \\
\hline $1.50 \mathrm{~d}$ & $\mathrm{Br}^{82}$ & $\beta^{-} .46$ & $\begin{array}{r}.55, .77,1 . \alpha, \\
.6-2\end{array}$ & E 3.1 \\
\hline $1.50 \mathrm{~d}$ & $\mathrm{Cf}^{246}$ & $\begin{array}{l}a 6.75,6.71, \ldots \\
S F \\
e^{-}\end{array}$ & $\gamma .044, .10, .15$ & M 246.146 \\
\hline $1.50 \mathrm{~d}$ & $\mathrm{Ni} 57$ & $\beta^{+} .84$ & 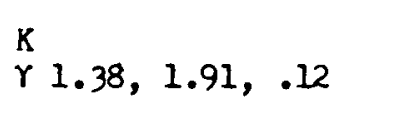 & E 3.24 \\
\hline $1.50 \mathrm{~d}$ & $\mathrm{Rh}^{105}$ & $\beta^{-} .25, .56$ & $r .31, \cdots$ & E. .56 \\
\hline $1.54 \mathrm{~d}$ & $E^{254}$ & $\beta^{-} 1.1, \cdots$ & $\begin{array}{l}K \\
\gamma .68\end{array}$ & \\
\hline $1.58 \mathrm{~d}$ & $\mathrm{Eu}^{146}$ & $e^{-} \cdot 4$ & $\mathrm{~K}$ & \\
\hline $1.58 \mathrm{~d}$ & $S b^{119}$ & $e^{-}$ & $\stackrel{K}{r} . \circledast 4 D$ & E. .59 \\
\hline $1.62 \mathrm{~d}$ & As 77 & $\beta^{-} .69, \cdots$ & $\begin{array}{l}r .24, .52, .086, \\
.160, . .\end{array}$ & E.69 \\
\hline $1.62 \mathrm{~d}$ & $\mathrm{Au}^{194}$ & $\begin{array}{l}\beta^{+} 1.55,1.22 \\
e^{-}\end{array}$ & $\begin{array}{l}K \\
\gamma .33,1.48, .29-2.1\end{array}$ & E 2.57 \\
\hline $1.62 \mathrm{~d}$ & $\mathrm{Ba} 133$ & $e^{-}$ & I.T. .276 & \\
\hline $1.67 \mathrm{~d}$ & $\mathrm{Hg}^{195}$ & $e^{-}$ & $\begin{array}{l}K \\
I . T . .123 \\
r .26, . \alpha_{4}-.56\end{array}$ & \\
\hline
\end{tabular}

* Total Disintegration Energy (Mev), or Isotopic Mass

Page 37. 


\begin{tabular}{|c|c|c|c|c|}
\hline 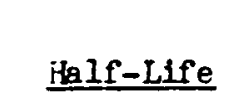 & Isotope & $\begin{array}{l}\text { Particles and } \\
\text { Energies (Mev) }\end{array}$ & $\begin{array}{l}\text { Gamma Energies } \\
(\mathrm{Mev})\end{array}$ & $\begin{array}{l}\text { Total En } \\
\text { or Mes } \\
\end{array}$ \\
\hline $1.67 \mathrm{~d}$ & $G e^{69}$ & $\beta^{+} 1.21, .6, \cdots$ & $\begin{array}{l}\mathrm{k} \\
\mathrm{r} \\
.09-1.12, .58, .87\end{array}$ & \\
\hline $1.67 \mathrm{~d}$ & $L_{A} 140$ & $\beta^{-} 1.34, .8-2.15$ & $\gamma 1.60, .11-2.9$ & E 3.75 \\
\hline $1.71 \mathrm{~d}$ & $\operatorname{Ir} 188$ & $\frac{\beta+2}{e^{+}} \ldots$ & $\frac{k}{r .1550, .63,48, \cdots}$ & $\cdots+$ \\
\hline $1.7 \mathrm{~d}$ & $\operatorname{Lu} 170$ & $0^{-} .1$ & $\begin{array}{l}K \\
r \sim 2.5\end{array}$ & \\
\hline $1.8 \mathrm{~d}$ & $\mathrm{Bk}^{246}$ & & ${ }_{i .1}^{K} .80, .145, .98,1.0$ & \\
\hline $1.83 \mathrm{~d}$ & $\mathrm{Sc}^{48}$ & $\beta^{-} .64$ & $r 1.32,1.04, .99$ & E 4.0 \\
\hline $1.96 \mathrm{~d}$ & $\mathrm{Am}^{240}$ & & $\begin{array}{l}K \\
r 1.02,1.40, .92\end{array}$ & \\
\hline $1.96 \mathrm{~d}$ & $\mathrm{Sm}^{153}$ & $\beta^{-} .71, .64, .81, \cdots$ & $r .102 D, .069 D, .08-$ & E. .81 \\
\hline$\sim 2 \mathrm{~d}$ & $\operatorname{Tn} 174$ & $\beta^{-}$ & & \\
\hline $2 \cdot \alpha_{+} d$ & $\ln 72$ & $\beta^{-} .3,1.6$ & $\gamma$ & \\
\hline $2.08 \mathrm{~d}$ & $\operatorname{Pm}^{149}$ & $\beta^{-1.05}$ & $r .29,1.3$ & E 1.34 \\
\hline $2.10 \mathrm{~d}$ & $\mathrm{~Np}^{238}$ & $\beta^{-1.26,} .27, \cdots$ & $r .044, .99,1.03, \ldots$ & E 1.30 \\
\hline $2.17 \mathrm{~d}$ & $\mathrm{~Pb}^{203}$ & & $\begin{array}{l}K \\
Y .28, .40, .68\end{array}$ & E 1.4 \\
\hline $2.2 \mathrm{~d}$ & $\mathrm{Ta} I 77$ & $e^{-} .1$ & $\begin{array}{l}K \\
r \sim 1.4\end{array}$ & \\
\hline $2.2 \mathrm{~d}$ & $\operatorname{Re}^{184}$ & $e^{-}$ & $\begin{array}{l}\mathrm{k} \\
\mathrm{r} \cdot \alpha_{4}, .16\end{array}$ & \\
\hline $2.26 \mathrm{~d}$ & $\operatorname{cd}^{115}$ & $\beta^{-} 1.11, \cdots$ & $\gamma .52, \cdots,(.34)$ & E 1.45 \\
\hline $2.3 d$ & $\mathrm{Xe}^{133}$ & $e^{-}$ & I.T. . .233 & : \\
\hline $2.33 d$ & $N p^{239}$ & $\beta^{-} .33-.72$ & $\begin{array}{c}r .0610, .105 \mathrm{D}, \\
.045-.49\end{array}$ & E. .72 \\
\hline
\end{tabular}

* Total Disintegration Energy (Mev), or. Isotopic Mass 


\begin{tabular}{|c|c|c|c|c|}
\hline Half-Life & Isotope & $\begin{array}{l}\text { Particles and } \\
\text { Energies (Mev) }\end{array}$ & $\begin{array}{l}\text { Gamma Energies } \\
\frac{(\mathrm{MeV})}{}\end{array}$ & $\begin{array}{l}\text { Total Ene } \\
\text { or Mass } \\
\end{array}$ \\
\hline $2.36 d$ & $N_{1}^{66}$ & $\beta^{-} .3$ & & \\
\hline $2.37 \mathrm{~d}$ & $\operatorname{Br}^{77}$ & $\beta^{+} .34$ & $\stackrel{K}{\gamma} \underset{(.16)}{.52, .086-1.0,}$ & E 1.36 \\
\hline $2.4 \mathrm{~d}$ & Sct44 & $e^{-}$ & I.T. .271 & \\
\hline $2.4 d$ & $\mathrm{Ba}^{128}$ & $(\beta+3.0, \cdots)$ & $\begin{array}{l}K \\
r .27,(.13-.98)\end{array}$ & \\
\hline $2.54 \mathrm{~d}$ & $\mathrm{Cu}^{67}$ & $\begin{array}{l}\beta^{-} .40, .48, .58 \\
\theta^{-}\end{array}$ & $r .182, .090, .092 \mathrm{D}$ & E .58 \\
\hline $2.58 \mathrm{~d}$ & $\mathrm{As}^{71}$ & $\frac{\beta^{+}}{\theta^{-}} .81, \cdots$ & $r .175, . .03$ & \\
\hline $2.67 \mathrm{~d}$ & $R_{e} 182$ & $e^{-}$ & $\begin{array}{l}K \\
Y .11-.35,1.5\end{array}$ & \\
\hline $2.67 \mathrm{~d}$ & $Y^{90}$ & $\begin{array}{l}\beta^{-} 2.27, \cdots \\
\theta^{-}-1.7\end{array}$ & & E 2.27 \\
\hline $2.70 \mathrm{~d}$ & 197 & $e^{-}$ & $\begin{array}{l}\mathrm{K} \\
\mathrm{r} .077 \mathrm{D}, .191\end{array}$ & \\
\hline $2.70 \mathrm{~d}$ & $\mathrm{Au}^{198}$ & $\beta^{-} .96, .28,1.37$ & $r .4118 D, .68,1.09$ & E 1.37 \\
\hline $2.79 \mathrm{~d}$ & Mo99 & $\beta-1.23, .45, \cdots$ & $\gamma(.78), .74, . \alpha_{4}-$ & $E 1.37$ \\
\hline $2.8 \mathrm{~d}$ & In 111 & $e^{-}$ & $\begin{array}{l}\mathrm{K} \\
\gamma .247 \mathrm{D}, .172, .33, \\
\quad .09,(.150)\end{array}$ & \\
\hline $2.8 \mathrm{~d}$ & $\mathrm{sb}^{122}$ & $\begin{array}{l}\beta-1.4,1.98, .73 \\
\beta+.5\end{array}$ & $\begin{array}{l}K \\
\gamma .56, .7-1.2\end{array}$ & $\begin{array}{l}E^{-} 1.98 \\
E^{+} 1.5\end{array}$ \\
\hline $2.9 \mathrm{~d}$ & $\operatorname{Pau}^{97}$ & $e^{-}$ & $\begin{array}{l}K \\
\gamma .22, . .33, .57, . .11\end{array}$ & \\
\hline $3 \cdot d$ & $\mathrm{Ce}^{13 / 4}$ & & $\begin{array}{l}\text { K } \\
\text { no } r\end{array}$ & \\
\hline $3 d$ & $\mathrm{Fm}^{253}$ & & $\mathbf{K}$ & \\
\hline $3.0 \mathrm{~d}$. & $\mathrm{T}^{201}$ & $e^{-}$ & $\begin{array}{l}\mathrm{K} \\
\gamma .167, .135, .032 \\
.031\end{array}$ & \\
\hline
\end{tabular}

* Total Disintegration Energy (Mev), or Isotopic Mass 


\begin{tabular}{|c|c|c|c|c|}
\hline Helf-Lfe & Isotope & $\begin{array}{l}\text { Particles and } \\
\text { Energies (Mov) }\end{array}$ & $\begin{array}{c}\text { Gamma Energies } \\
\text { (Mev) } \\
\end{array}$ & $\begin{array}{l}\text { Total Energy } \\
\text { or Mass* }\end{array}$ \\
\hline $3.0 \mathrm{~d}$ & Pt ${ }^{191}$ & $\theta^{-}$ & $\begin{array}{l}K \\
\gamma .129, .042, .54, \\
.047-.63\end{array}$ & \\
\hline $3.15 \mathrm{~d}$ & $\mathrm{Au}^{199}$ & $\beta^{-} .30, .25, .46$ & $r .158 \mathrm{D}, .208 \mathrm{D}, .0500$ & E .46 \\
\hline $3.21 \mathrm{~d}$ & $\mathrm{Te}^{132}$ & $\begin{array}{l}\beta^{-} .22, \cdots \\
\left(\beta^{-} .9-2.1\right)\end{array}$ & $\begin{array}{l}r .23, \cdots \\
-(\gamma .67-2.2)\end{array}$ & \\
\hline $3.25 \mathrm{~d}$ & $\mathrm{Ga}_{a} 67$ & $e^{-}$ & $\begin{array}{l}\mathrm{K} \\
\mathrm{Y} .092 \mathrm{D}, .18, .30 \\
.090-.88\end{array}$ & E 1.00 \\
\hline $3.3 \mathrm{~d}$ & $\mathrm{Na}^{140}$ & & K & E. 1 \\
\hline $3.34 \mathrm{~d}$ & $Y^{87}$ & $\beta^{+} .7$ & $\begin{array}{l}\mathrm{K} \\
\gamma .48,(.39)\end{array}$ & E 2.1 \\
\hline $3.4 \mathrm{~d}$ & $P t^{193}$ & & I.T. .135 & \\
\hline $3.4 \mathrm{~d}$ & $\operatorname{se} 47$ & $\beta^{-} .44, .60$ & $\gamma .16$ & E. .60 \\
\hline $3.42 \mathrm{~d}$ & $D_{y}^{166}$ & $\beta^{-} \cdot 3$ & $r<.05$ & $E \cdot 3$ \\
\hline $3.50 \mathrm{~d}$ & $\mathrm{Nb} 95$ & $\theta^{-}$ & I.T. .23 & \\
\hline $3.64 \mathrm{~d}$ & $\mathrm{Ra} 224$ & a $5.68,5.44$ & $r .24$ & M 224.090 \\
\hline $3.79 \mathrm{~d}$ & $R^{186}$ & $\beta-1.07, .93, \cdots$ & $\begin{array}{l}\mathrm{K} \\
\mathrm{r} .137 \mathrm{D}, .123, .76, \\
.63\end{array}$ & $E 1.07$ \\
\hline $3.825 \mathrm{~d}$ & $\operatorname{Em}^{222}$ & a 5.48 & & M 222.087 \\
\hline $3.89 \mathrm{~d}$ & $\mathrm{Sb}^{127}$ & $\beta^{-} .86,1.57,1.11$ & $r .67, .46, .24$, & \\
\hline $4.0 \mathrm{~d}$ & $\mathrm{Pd}^{100}$ & & $\mathrm{~K} .081,1.8 ?$ & \\
\hline $4.2 d$ & $Y^{175}$ & $\beta^{-} .47, .07, .36$ & $\begin{array}{l}r .40, .28, .113 \\
.14, . .\end{array}$ & E. .47 \\
\hline $4.3 \mathrm{~d}$ & $0^{231}$ & a 5.45 & $\stackrel{\mathrm{K}}{\mathrm{r} .051, .064, .076}$ & M 231.109 \\
\hline $4.3 d$ & $\mathrm{Tc}^{96}$ & & ${ }_{i .12}^{\mathrm{K}}, .77, .81$, & E 3.0 \\
\hline
\end{tabular}

- Total Disintegration Energy (Mev), or Isotopic Mass

Page 40. 


\begin{tabular}{|c|c|c|c|c|}
\hline Walf-LAfe & Isotope & $\begin{array}{l}\text { Particles and } \\
\text { Energles (Mev) }\end{array}$ & $\begin{array}{c}\text { Cemma Energies } \\
(\mathrm{Mev})\end{array}$ & $\begin{array}{l}\text { Total Er } \\
\text { or Mgs }\end{array}$ \\
\hline $4 \cdot 4 d$ & $\mathrm{~Np}^{234}$ & $\beta^{+} .8$ & $\begin{array}{l}K, L \\
\gamma .8,1.57, \ldots\end{array}$ & \\
\hline $4.5 \mathrm{~d}$ & $? R^{101}$ & $\theta^{-}$ & $\frac{K}{r} .30, .15, .56$ & \\
\hline $4.5 \mathrm{~d}$ & $I^{124}$ & $\beta+2.20, \cdots$ & $\stackrel{K}{r} .60,1.7,2.0, .7$ & E 3.22 \\
\hline $4.5 d$ & $\mathrm{~T}^{119}$ & $e^{-} .2, .5$ & $\begin{array}{l}K \\
r 1.6\end{array}$ & \\
\hline$\cdot 4.7 \mathrm{~d}$ & $\mathrm{Ca}^{47}$ & $\beta^{-} .7,2.0$ & $r 1.30, .81, .50$ & E 2.0 \\
\hline $4.8 \mathrm{~d}$ & $A u^{192}$ & $\beta+1.9$ & $\underset{i .16}{\mathrm{~K}}, .317, .296, .137-$ & - \\
\hline $5 d$ & $\mathrm{Eu}^{145}$ & $\theta^{-} .2$ & K & \\
\hline $5 d$ & $T b^{156}$ & $\beta^{-} ? .6, .2$ & $Y .10-2.0$ & \\
\hline $5.0 d$ & $\mathrm{Bk}^{245}$ & $\alpha 6.17,6.33,5.90$ & $\begin{array}{l}\mathrm{K} \\
\gamma .25, .38, .16\end{array}$ & \\
\hline $5.0 \mathrm{~d}$ & $\mathrm{B1}^{210}$ & $\beta^{-1.17}$ & & E 1.17 \\
\hline $5.1 \mathrm{~d}$ & $T^{153}$ & $0^{-} .15, \sim .3$ & $\begin{array}{l}\mathrm{K} \\
\mathrm{r} .23,1.2\end{array}$ & \\
\hline $5.2 \mathrm{~d}$ & $\mathrm{Ta}^{183}$ & $\beta^{-} .62, \cdots$ & $r .041-.41$ & $E 1.07$ \\
\hline $5.27 \mathrm{~d}$ & $x_{e} 133$ & $\mathrm{\beta}^{-} \cdot 35$ & $r .0810$ & E.43 \\
\hline $5.3 d$ & $\operatorname{Pn} 148$ & $\beta^{-}-2.5$ & $\gamma .8$ & \\
\hline $5.7 \mathrm{~d}$ & $\mathrm{Mn}^{52}$ & $\beta^{+} .6$ & $\frac{k}{r} 1.4, .9, .7$ & E 4.7 \\
\hline $5.8 \mathrm{~d}$ & $\mathrm{Sb}^{120}$ & $e^{-}$ & $\begin{array}{l}K \\
r \\
.09\end{array}$ & \\
\hline $6 d$ & Pt 195 & & $\begin{array}{l}\text { I.T. } .130 \\
\text { r.099, .031 }\end{array}$ & \\
\hline
\end{tabular}

- Total Disintegration Energy (Mov), or Isotopic Mass

Page 41. 


\begin{tabular}{|c|c|c|c|c|}
\hline Helf-Ifes & Isotope & $\begin{array}{l}\text { Particles and } \\
\text { Bnergles (Mov) }\end{array}$ & $\begin{array}{l}\text { Gamma Energies } \\
\frac{(\mathrm{MeV})}{}\end{array}$ & $\begin{array}{l}\text { Total Enersy } \\
\text { or Mas8 }\end{array}$ \\
\hline $6.0 \mathrm{~d}$ & $\mathrm{Te}^{118}$ & $\left(\beta^{+} 3.1\right)$ & $\stackrel{K}{(r .11)}$ & . \\
\hline $6.2 \mathrm{~d}$ & $\mathrm{Co}^{132}$ & & $\begin{array}{l}K \\
\gamma .67,1.27,1.10\end{array}$ & ' \\
\hline $6.4 \mathrm{~d}$ & $\mathrm{B1}^{206}$ & $\theta^{-}$ & $\begin{array}{l}\mathrm{K} \\
\mathrm{r} .800 ; .88 \mathrm{D}, .52 \mathrm{D}, \\
1.72, .107-1.60\end{array}$ & E 3.6 \\
\hline $6.4 \mathrm{~d}$ & $\mathrm{N1} 56$ & & $\frac{K}{\gamma} .16, .85, .26-1.74$ & \\
\hline $6.7 \mathrm{~d}$ & $\mathbf{w}^{172}$ & $0^{-} .13, \sim .6$ & $\begin{array}{l}K \\
Y 1.2\end{array}$ & \\
\hline $6.75 \mathrm{~d}$ & $0^{237}$ & $\mathrm{\beta}^{-} .24, \cdots$ & $r .21, .059, .027-.43$ & E. .51 \\
\hline $6.8 \mathrm{~d}$ & $\mathbf{L u} 177$ & $\beta^{-} .50, .18, .39$ & $r .113, .208, .07-.32$ & E. .50 \\
\hline $7 \mathrm{~d}$ & To161 & $\beta^{-} .55, \cdots$ & $r .049, .08$ & E. 6 \\
\hline $7.5 \mathrm{~d}$ & $\Delta B^{111}$ & $\beta^{-} 1 . \alpha_{4}, .7, \cdots$ & $r .34, .2470$ & E $1 . \alpha_{4}$ \\
\hline $8 \mathrm{~d}$ & $X_{\theta} 129$ & $0^{-}$ & $\begin{array}{l}\text { I.T. } \\
r \cdot 040\end{array}$ & \\
\hline $8 d$ & $\mathrm{Rb}<81$ & & & \\
\hline $8.05 d$ & $I^{131}$ & $\beta^{-} .61, .25-.81$ & $r .36, .0800-.72$ & E .97 \\
\hline $8.3 d$ & $\Delta 8^{206}$ & & $\begin{array}{l}K \\
\gamma .51, .22-2.66\end{array}$ & . \\
\hline $8.5 \mathrm{~d}$ & Lu171 & $0-.17, .5$ & $\begin{array}{l}K \\
r \sim 1.2\end{array}$ & \\
\hline $9 d$ & $P O^{206}$ & a $5.22, \cdots$ & $\begin{array}{l}K \\
r . \infty 0-1.03\end{array}$ & M 206.046 \\
\hline $9 d$ & $\operatorname{cdd}^{149}$ & $\begin{array}{l}\theta^{-} .3 \\
\alpha 3.0\end{array}$ & $\mathrm{~K}$ & \\
\hline $9.4 \mathrm{~d}$ & $\operatorname{Br}^{169}$ & $\beta^{-} .33$ & no $r$ & E. .33 \\
\hline $9.6 \mathrm{~d}$ & $\operatorname{Tm} 167$ & . & $\begin{array}{l}K \\
r .20, .72, .51, .11\end{array}$ & \\
\hline
\end{tabular}

- Total Dlsintegration Bnergy (Mev), or Isotopic

Page 42. 


\begin{tabular}{|c|c|c|c|c|}
\hline 地保-Ife & Isotope & $\begin{array}{l}\text { Particles and } \\
\text { Energies (Mev) }\end{array}$ & $\begin{array}{l}\text { Cemma Energies } \\
\text { (Mer) }\end{array}$ & $\begin{array}{l}\text { Total Energo } \\
\text { or Mas: }\end{array}$ \\
\hline $9.7 \mathrm{~d}$ & $\operatorname{Ir} 196$ & $\beta^{-} .08$ & $r .58, .76, \sim 1$ & \\
\hline $9.7 d$ & $\mathrm{Cs} 131$ & & $K, L$ & E. 35 \\
\hline $9.7 \mathrm{~d}$ & $\mathrm{Se}^{72}$ & & $\mathbf{K}$ & \\
\hline $10 d$ & $\mathrm{Sn}^{125}$ & $\beta-2.4, \cdots$ & $r \cdot 33-2.0$ & E 2.4 \\
\hline $10 d$ & $\mathrm{NB}^{92}$ & & $\begin{array}{l}K \\
r .93,1.83, .90\end{array}$ & E 1.9 \\
\hline $10 \mathrm{~d}$ & Pt 188 & & $\begin{array}{l}K \\
\gamma .192, .28, .043-.4\end{array}$ & . \\
\hline$\sim 10 \mathrm{~d}$ & $\mathrm{Sr}^{89}$ & & $\underset{r}{r} \cdot \mathrm{T}$ & \\
\hline $10.0 \mathrm{~d}$ & $A c^{225}$ & a 5.80 & $r .085$ & M 225.093 \\
\hline $11 d$ & $\operatorname{Ir} 190$ & & $\begin{array}{l}K \\
\gamma .19, .36-1.3\end{array}$ & \\
\hline $11 \mathrm{~d}$ & $\mathrm{Pu}^{246}$ & $\beta^{-} .15, \cdots$ & $r .10, .22, .043, .18$ & \\
\hline $11 d$ & $\operatorname{Ir}{ }^{189}$ & & $\begin{array}{l}k \\
r \\
.24, \cdots\end{array}$ & \\
\hline $11.6 \mathrm{~d}$ & $\mathrm{Ra}^{223}$ & $\begin{array}{l}a .70,5.60,5.42- \\
5.86\end{array}$ & $r .026-.44$ & M 223.088 \\
\hline $11.6 \mathrm{a}$ & $\mathrm{Nd}^{247}$ & $\beta^{-} .81, .37, \cdots$ & $r .092, .53, .12-.69$ & E.90 \\
\hline $11.6 \mathrm{~d}$ & $\mathrm{Ba}^{131}$ & & $\begin{array}{l}K \\
r \\
.040, .122 D, .214 \\
.04-1.03\end{array}$ & \\
\hline $12 \mathrm{~d}$ & $x_{\theta} 131$ & $\theta^{-}$ & I.T. .164 & \\
\hline $12 d$ & $\mathrm{Tl}^{20 R}$ & $\theta^{-}$ & $\begin{array}{l}K, L \\
r .440\end{array}$ & \\
\hline $12 \mathrm{~d}$ & $c_{\theta}^{71}$ & & $\mathbf{K}$ & E. .23 \\
\hline $12.8 \mathrm{~d}$ & $\mathrm{Ba}^{1} \mathrm{~K}_{\mathbf{O}}$ & $\beta^{-1.02, .48,(1.3-2.3)}$ & $\underset{(1.6,1-2.5)}{r .54, .31, .16, \cdots}$ & \\
\hline $13 d$ & $\mathrm{Cs}^{136}$ & $\beta^{-} .34, .66$ & $r 1.04, .82, .067-2.5$ & \\
\hline
\end{tabular}

* Total Disintegration Energy (Mev), or Isotople Mass 


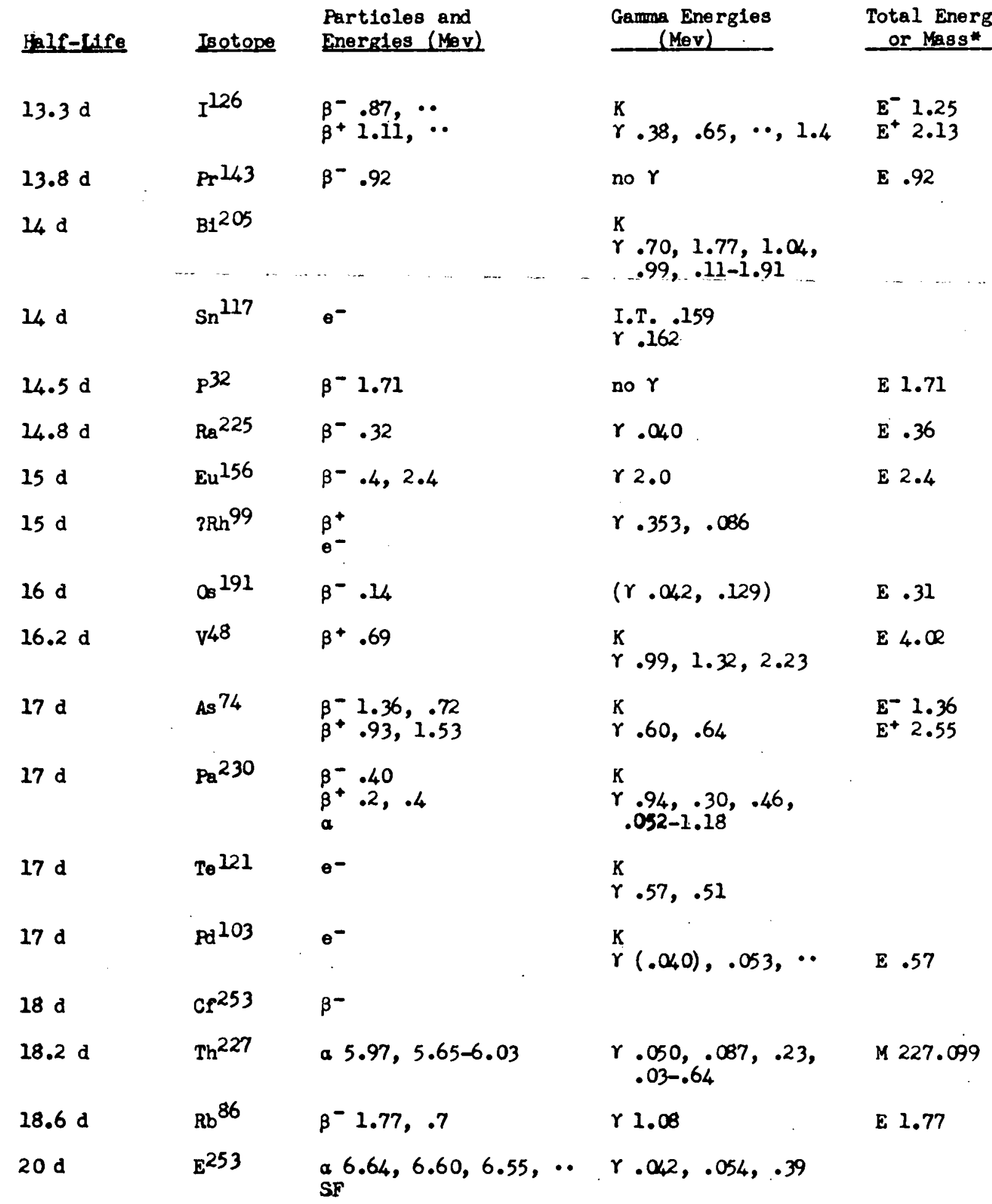

- Total Disintogration Energy (Mev), or Isotopic Mass

Page 44. 


\begin{tabular}{|c|c|c|c|c|}
\hline 蝈If-Lffe & Isotope & $\begin{array}{l}\text { Particles and } \\
\text { Energies (Mev) }\end{array}$ & $\begin{array}{c}\text { Gamma Energies } \\
(\mathrm{MeV})\end{array}$ & $\begin{array}{l}\text { Total Energy } \\
\text { or Mass* }\end{array}$ \\
\hline $21 d$ & $U^{230}$ & a $5.89,5.82,5.66$ & $\gamma .07, .16, .23$ & M 230.106 \\
\hline $21 d$ & $w^{178}$ & & $\begin{array}{l}K \\
r \sim \cdot 3\end{array}$ & \\
\hline $24 \mathrm{~d}$. & $\mathrm{Eu}^{1 / 47}$ & $\begin{array}{l}\theta^{-} \\
\alpha 2.2\end{array}$ & $\begin{array}{l}K \\
Y .21, .12\end{array}$ & \\
\hline $24.10 d$ & $\mathrm{Th}^{234}$ & $\beta^{-} .19, .10$ & $r .093, .064, .089$ & E.19 \\
\hline $25 d$ & $p^{33}$ & $\beta^{-} .25^{\circ}$ & no $r$ & E. .25 \\
\hline $26 d$ & $\mathrm{Sr}^{82}$ & $\left(\beta^{+} 3.2\right)$ & $\mathrm{K}$ & \\
\hline $27 d$ & $\mathrm{Cr}^{51}$ & & $\begin{array}{l}\mathrm{K} \\
\mathrm{r} \cdot 32\end{array}$ & E. .75 \\
\hline $27 d$ & $\mathrm{Cm}^{240}$ & $\begin{array}{l}\text { a } 6.25 \\
\text { SF }\end{array}$ & & M 240.131 \\
\hline $27.4 \mathrm{~d}$ & $\mathrm{~Pa}^{233}$ & $\beta^{-} .26, .14, .57$ & $\begin{array}{l}r .086, .31, .075 \\
.016-.42\end{array}$ & E .57 \\
\hline $28 d$ & $\mathrm{Sb}^{126}$ & $\beta^{-} 1.9$ & & \\
\hline$\sim 30 \mathrm{~d}$ & $E^{255}$ & $\beta^{-}$ & & \\
\hline $32 \mathrm{~d}$ & $\mathrm{Ce}^{1 / 4 \mathrm{l}}$ & $\beta^{-} .43, .57$ & $\gamma .145 D$ & E ..57 \\
\hline $32 \mathrm{~d}$ & $Y b^{169}$ & $e^{-}$ & $\underset{.308 \mathrm{D}}{\mathrm{K}} \underset{.004, .008 \mathrm{D}, .094-}{ }$ & \\
\hline $33 d$ & $\mathrm{Te}^{129}$ & $e^{-}$ & I.T. .106 & \\
\hline $33 d$ & $\mathrm{Rb}^{84}$ & $\begin{array}{l}\beta+1.7, \cdots \\
\beta-1\end{array}$ & $\begin{array}{l}K \\
\gamma .89-1.9\end{array}$ & $\begin{array}{l}E^{-} \cdot 4 \\
E^{+} 2.7\end{array}$ \\
\hline $35 \mathrm{~d}$ & $\mathrm{Cm}^{24 \mathrm{I}}$ & a 5.95 & $\begin{array}{l}K \\
\gamma .47, .59, . .\end{array}$ & \\
\hline $35 d$ & $A^{37}$ & & $\mathrm{~K}, \mathrm{~L}$ & E. .82 \\
\hline $35 d$ & $\mathrm{Nb} 95$ & $\beta^{-} .16$ & $r .76, \cdots$ & E. .92 \\
\hline $36.4 \mathrm{~d}$ & $\mathrm{Xe}^{127}$ & & $\begin{array}{l}K \\
Y .203, .057-.36\end{array}$ & \\
\hline
\end{tabular}

* Total Disintegration Energy (Mev), or Isotopic Mass 


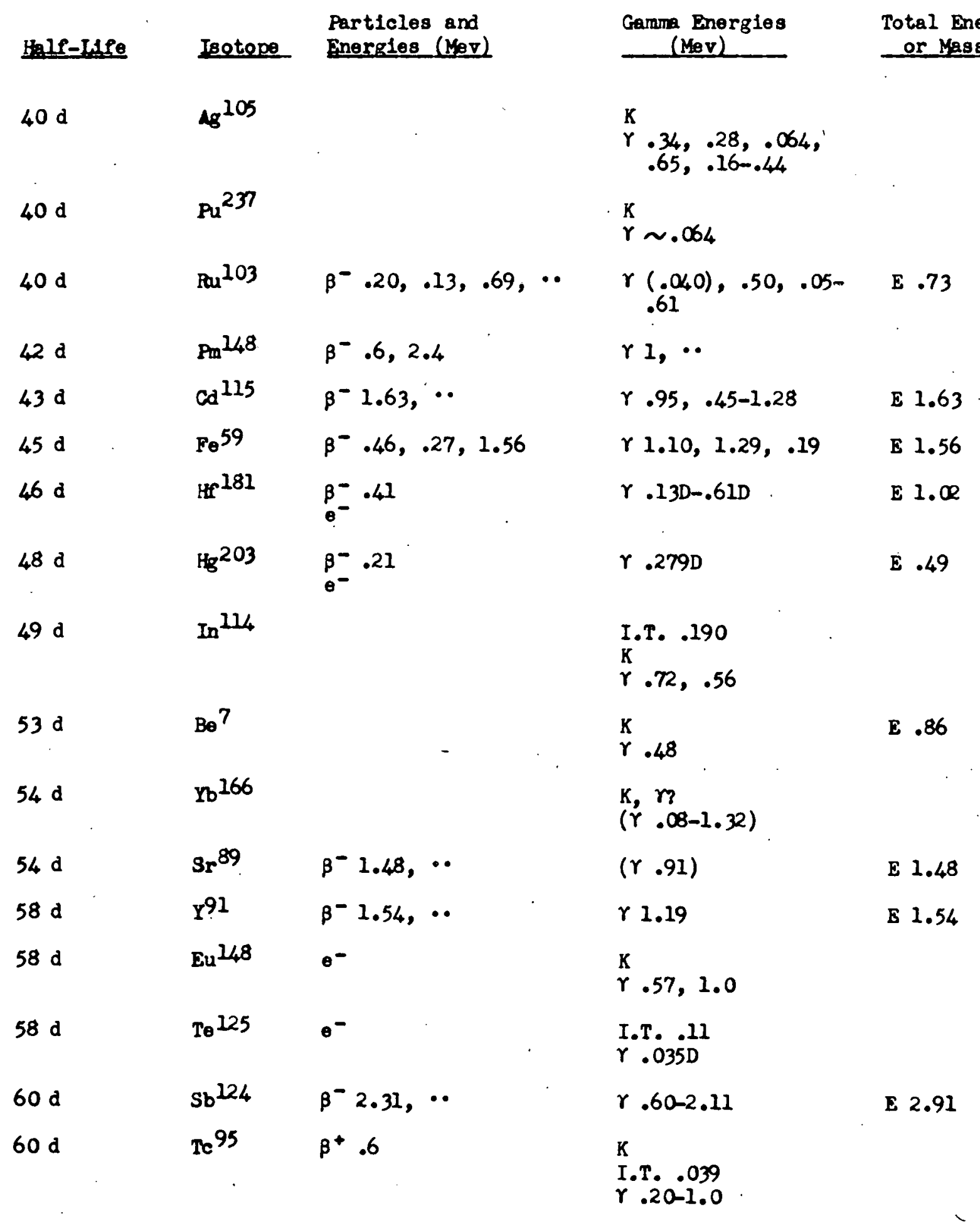

- Total Disintegration Fnergo (Nev), or Isotopic Mass

Page 46. 


\begin{tabular}{|c|c|c|c|c|}
\hline 焦保-Le & Isotope & $\begin{array}{l}\text { Particles and } \\
\text { mergles (Mev) }\end{array}$ & $\begin{array}{c}\text { Ganma Energles } \\
\text { (Mav) }\end{array}$ & $\begin{array}{l}\text { Total Energ } \\
\text { or Nas8* } \\
\end{array}$ \\
\hline $60 \mathrm{~d}$ & $I^{125}$ & & $\begin{array}{l}K, L \\
r .035\end{array}$ & E. .15 \\
\hline $62 d$ & $\mathrm{Nb} 91$ & $\theta^{-}$ & $\begin{array}{l}\mathrm{I} . T . .105 \\
\mathrm{~K} \\
\mathrm{Y} 1.19\end{array}$ & \\
\hline $65 d$ & $w^{188}$ & $\beta^{-}$ & & \\
\hline $65 d$ & $2 r^{95}$ & $\beta_{\left(0^{-}\right)}^{-} \cdot 36, .39, .88$ & $\gamma .75, .72,(.23)$ & E 1.12 \\
\hline $65 d$ & $s r^{85}$ & & $\begin{array}{l}K \\
\gamma .510\end{array}$ & \\
\hline $70 d$ & $\mathrm{Cf}^{254}$ & SP & & \\
\hline $70 \mathrm{~d}$ & $\mathrm{Hr}^{175}$ & . & $\begin{array}{l}K \\
r .343, .089, .113- \\
.432\end{array}$ & \\
\hline $71 \mathrm{~d}$ & $\mathrm{Co}^{58}$ & $\beta^{+} .48, \cdots$ & $\stackrel{K}{r} .81,1.64$ & B 2.31 \\
\hline $72 d$ & $T^{260}$ & $\beta^{-} .56, .85, \cdots$ & $r .96, .086 \mathrm{D}-1.45$ & E 1.81 \\
\hline $74 d$ & $\operatorname{Ir} 192$ & $\beta^{-} .67, \cdots$ & $\mathrm{K}_{i .31,}^{K}, 47, .136-$ & E 1.58 \\
\hline $74 \mathrm{~d}$ & $w^{185}$ & $\beta^{-} .43, \cdots$ & $r .056$ & $E \cdot 43$ \\
\hline $76 d$ & $A s 73$ & $\left(\theta^{-1}\right)$ & $\stackrel{K}{r} .054, .023 D)$ & E.37 \\
\hline $77 \mathrm{~d}$ & $\mathrm{Co}^{56}$ & $\beta^{+} 1.50, \cdots$ & $\stackrel{K}{r} .85,1.2,1.7-3.2$ & B 4.62 \\
\hline $83 d$ & $\mathrm{Rb}^{83}$ & & $\begin{array}{l}K \\
r \\
(r .52 \\
r .032, .09)\end{array}$ & \\
\hline $85 d$ & $\mathrm{zr}^{88}$ & $e^{-}$ & 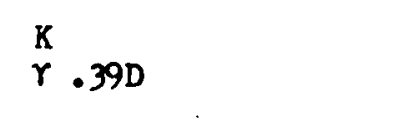 & \\
\hline $85 d$ & $\operatorname{Sc}^{46}$ & $\beta^{-} \cdot 36, \cdots$ & $r 1.12, .88, \ldots$ & E 2.36 \\
\hline
\end{tabular}

- Total Dis Integration Energy (Mev), or Isotopic Mass

Page 47. 


\begin{tabular}{|c|c|c|c|c|}
\hline ple-Lifo & Isotope & $\begin{array}{l}\text { Particles and } \\
\text { Energies (Nav) }\end{array}$ & $\begin{array}{l}\text { Camma Energles } \\
\frac{(\mathrm{Mev})}{}\end{array}$ & $\begin{array}{l}\text { Total Energa } \\
\text { or Mags* }\end{array}$ \\
\hline $87 d$ & $\mathrm{Tm}^{168}$ & $\begin{array}{l}\theta^{-} \\
\beta^{-} .5(3)\end{array}$ & $\begin{array}{l}K \\
r .8, .20\end{array}$ & \\
\hline $87 d$ & $\mathrm{~s}^{35}$ & $\beta^{-} .167$ & no $r$ & E. .167 \\
\hline $91 \mathrm{~d}$ & $\mathrm{Tc}^{97}$ & $e^{-}$ & $\begin{array}{l}\text { I.T. . .099 } \\
r .090, \cdots\end{array}$ & \\
\hline $95 d$ & $a^{185}$ & & $\begin{array}{l}K, \mathrm{~L} \\
r .65, .88, .23, \\
.16, . .\end{array}$ & r \\
\hline $1 \alpha_{4} \mathrm{~d}$ & $T_{0}^{123}$ & $e^{-}$ & $\begin{array}{l}\text { I.T. } .0088 \\
\text {. } .159 D\end{array}$ & \\
\hline $110 \mathrm{~d}$ & $\mathrm{Te}^{127}$ & $e^{-}$ & I.T. .008 & \\
\hline $112 \mathrm{~d}$ & $\mathrm{Ta}^{182}$ & $\beta^{-} .51, \cdots$ & $r 1.1,1.2, \ldots$ & E 1.7 \\
\hline $112 \mathrm{~d}$ & $\mathrm{Sn}^{113}$ & $\left(\theta^{-}\right)$ & $\begin{array}{l}K, L \\
r .26 \\
(r \cdot 39)\end{array}$ & \\
\hline $120 d$ & $\mathrm{Bu} 149$ & $\theta^{-}$ & $\begin{array}{l}K \\
r .29, .57\end{array}$ & \\
\hline $127 \mathrm{~d}$ & $S_{\theta} 75$ & & $\begin{array}{l}K \\
r .27, .14,(.28), \\
.066-.40\end{array}$ & \\
\hline $129 d$ & $\operatorname{Tm}^{170}$ & $\beta_{-}^{-} .97, .89$ & $r .0840$ & E.97 \\
\hline $130 d$ & $\mathrm{Sn}^{123}$ & $\beta^{-} 1.42$ & & \\
\hline$\sim 130 \mathrm{~d}$ & $\mathrm{Hg}^{194}$ & & K & \\
\hline $134 \mathrm{~d}$ & $D y^{159}$ & & $\mathrm{~K}, \mathrm{~L}$ & \\
\hline $138.40 \mathrm{~d}$ & $\mathrm{PO}^{210}$ & a $5.30, \ldots$ & $\gamma .80$ & M 210.049 \\
\hline $140 \mathrm{~d}$ & $\mathrm{Ce}^{139}$ & $e^{-}$ & $\begin{array}{l}K \\
r .165 D\end{array}$ & E. 3 \\
\hline $140 \mathrm{~d}$ & $W^{181}$ & & $\begin{array}{l}\mathrm{K}, \mathrm{L} \\
\mathrm{r} .137, .153\end{array}$ & E . 9 \\
\hline $150 d$ & $R e^{183}$ & $e^{-}$ & 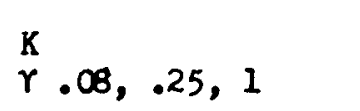 & \\
\hline
\end{tabular}

- Total D1sintegration Energy (Kev), or Isotopic Mass

Page 48. 


\begin{tabular}{|c|c|c|c|c|}
\hline Lelf-L1fe & Isotope & $\begin{array}{l}\text { Particles and } \\
\text { Energies (Mev) }\end{array}$ & $\begin{array}{l}\text { Ganma Energies } \\
\text { (MeV) }\end{array}$ & $\begin{array}{l}\text { Total Energy } \\
\text { or Mass* }\end{array}$ \\
\hline $150 \mathrm{~d}$ & $\mathrm{Te}^{121}$ & $e^{-}$ & $\begin{array}{l}I . T . \\
r .213^{.082}\end{array}$ & \\
\hline$\sim 150 \mathrm{~d}$ & $\mathrm{Cd}^{151}$ & $e^{-}$ & $\begin{array}{l}K \\
r .26\end{array}$ & \\
\hline$\sim 150 \mathrm{~d}$ & $\mathbf{E}^{252}$ & $\propto 6.64$ & & \\
\hline $160 \mathrm{~d}$ & $\mathrm{Ca} 45$ & $\beta^{-} .25$ & no $r$ & E. .25 \\
\hline $163 d$ & $\mathrm{Cm}^{242}$ & $\underset{S F}{a} 6.11,6.07, \cdots$ & $\gamma . \alpha_{4} 4, .10, .157$ & M 242.135 \\
\hline $165 \mathrm{~d}$ & $\mathrm{Lu} 174$ & $\beta^{-} .6$ & $\begin{array}{l}K \\
Y \sim 1\end{array}$ & \\
\hline $180 \mathrm{~d}$ & $A u^{195}$ & $e^{-}$ & $\begin{array}{l}K \\
r .099, .031, .14\end{array}$ & E. .27 \\
\hline$\sim 200 \mathrm{~d}$ & Ree 189 & $\beta^{-} .2$ & $r 1$ & \\
\hline $220 \mathrm{~d}$ & $R h^{102}$ & $\beta-1.15,1.24, \ldots$ & $\stackrel{K}{r .48, .20,1.1}$, & $\begin{array}{l}\mathrm{E}^{-1.15} \\
\mathrm{E}^{+} 2.26\end{array}$ \\
\hline $225 d$ & $C f^{248}$ & $\begin{array}{l}a 6.26 \\
S F\end{array}$ & & M 248.150 \\
\hline $236 d$ & $\operatorname{cd} 153$ & $e^{-}$ & 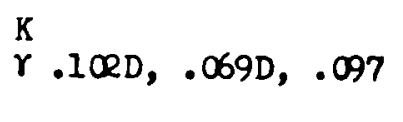 & \\
\hline $245 \mathrm{~d}$ & $2 n^{65}$ & $\beta^{+} .33$ & $\begin{array}{l}K \\
r 1.12\end{array}$ & E 1.35 \\
\hline $250 \mathrm{~d}$ & $\mathrm{Ge}^{68}$ & & $\mathbf{K}$ & \\
\hline $267 d$ & $\mathrm{Co}^{57}$ & $\theta^{-}$ & $\stackrel{K}{\gamma}: 123, .0240, .137$, & E.5 \\
\hline $270 \mathrm{~d}$ & Ag 110 & $\beta^{-} .53, .1, \cdots$ & $\begin{array}{l}\text { I.T. } .12 \\
r .66, .89, .93-2\end{array}$ & \\
\hline $275 d$ & $s n^{119}$ & $e^{-}$ & I.T. .065 & \\
\hline $285 d$ & $\mathrm{Co}^{1444}$ & $\beta^{-} \cdot 30, \cdots,(2.98, \cdots)$ & $r .03-1.4(.70-2.18)$ & E. 30 \\
\hline $290 d$ & $\mathrm{Bk}^{249}$ & $\begin{array}{l}\beta^{-} .09 \\
\alpha 5.40,5.08 \\
S F\end{array}$ & $r .32$ & \\
\hline
\end{tabular}

* Total Disintegration Energy (Mov), or Isotopic Mass 


\begin{tabular}{|c|c|c|c|c|}
\hline Hafe-Lfe & Isotope & $\begin{array}{l}\text { Particles and } \\
\text { Energtes (Nov) }\end{array}$ & $\begin{array}{c}\text { Gamma Energies } \\
(\mathrm{NeV})\end{array}$ & $\begin{array}{l}\text { Total mercy } \\
\text { or Naso }\end{array}$ \\
\hline $300 \mathrm{~d}$ & $\operatorname{Mn} 54$ & . & $\begin{array}{l}K \\
r .84\end{array}$ & E 1.38 \\
\hline$\sim 300 \mathrm{~d}$ & $\mathrm{Pm}^{143}$ & & $\begin{array}{l}K \\
r .9\end{array}$ & . \\
\hline$\sim 300 \mathrm{~d}$ & $\operatorname{Pm} 144$ & $-\cdots \cdots+\cdots$ & $-r .7,-4, \cdot 2,-$ & $\cdots+$ \\
\hline$\sim 1 g$ & $\mathrm{E}^{254}$ & $\begin{array}{l}\text { a } 6.44 \\
S F\end{array}$ & & M 254.168 \\
\hline$\sim 1 \mathrm{~g}$ & $v^{49}$ & . & $\mathbf{K}$ & E. .62 \\
\hline $1.0 \mathrm{~g}$ & $\mathrm{Sm}^{145}$ & . & $\begin{array}{l}K \\
r .061\end{array}$ & \\
\hline $1.0 \mathrm{~g}$ & $\operatorname{Pan} 106$ & $\beta^{-} . \alpha_{4},(3.53, \cdots)$ & $(r .51, .62-2.4)$ & E. . $\alpha_{4}$ \\
\hline$>1 \mathrm{~g}$ & $\mathrm{sn}^{121}$ & $\beta^{-} .42$ & & \\
\hline$\gg 1 \mathrm{~g}$ & $\mathrm{Cm}^{247}$ & & & \\
\hline $1.1 \mathrm{~g}$ & $\mathrm{~Np}^{235}$ & $\alpha 5.06$ & $\begin{array}{l}r, k \\
r .026, .085\end{array}$ & M 235.118 \\
\hline $1.3 \mathrm{y}$ & $\operatorname{cod}^{109}$ & $\left(0^{-}\right)$ & $\begin{array}{ll}K, L \\
(r & .087)\end{array}$ & E.15 \\
\hline $1.4 \mathrm{y}$ & $\mathrm{Lu}^{173}$ & $0^{-} .1, .2$ & $\begin{array}{l}K \\
r \sim .2, .8\end{array}$ & . \\
\hline $1.6 \mathrm{y}$ & $\mathrm{su}^{171}$ & $0^{-} .1$ & $\begin{array}{l}K \\
r \sim 1\end{array}$ & \\
\hline$\sim 1.6 \mathrm{~g}$ & $T 2179$ & $0^{-} .1$ & $K, Y .7$ & \\
\hline $1.7 \mathrm{~g}$ & Dul155 & $\beta^{-} .15, .25$ & $r .029-.137$ & E. .25 \\
\hline $1.9 \mathrm{y}$ & $\min 171$ & $\beta^{-} .10$ & no $r$ & $\mathrm{E} \cdot 10$ \\
\hline $1.90 \mathrm{~g}$ & $\mathrm{Th}^{228}$ & a $5.42,5.34, \cdots$ & $r .09, .21, .13, .17$ & M 228.100 \\
\hline$\sim 2 \mathrm{~g}$ & 0.394 & $\beta^{-}$ & 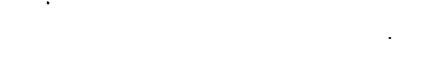 & \\
\hline$\sim 2 y$ & $\min ^{246}$ & $\beta^{-} \cdot 7$ & & \\
\hline
\end{tabular}

- Total D1sintegration Energy (Mev), or Isotople Mass 


\begin{tabular}{|c|c|c|c|c|}
\hline Helf-Lfo & Isotope & $\begin{array}{l}\text { Particlos and } \\
\text { Pnersiog (MeV) }\end{array}$ & $\begin{array}{l}\text { Gamma Fnergies } \\
\frac{(\mathrm{Ker})}{}\end{array}$ & $\begin{array}{l}\text { Total Her } \\
\text { or }\end{array}$ \\
\hline$>2 g^{-}$ & Ko93 & & $\mathbf{K}$ & E. .49 \\
\hline $2.2 \mathrm{y}$ & $c^{252}$ & $\begin{array}{l}a 6.11,6.07 \\
e^{-} \\
S F\end{array}$ & $\gamma .042, .100$ & \\
\hline $2.3 \mathrm{y}$ & 68134 & $\beta^{-} .65, \cdots$ & $r .60, .80, .47-1.37$ & E 2.05 \\
\hline $2.6 \mathrm{y}$ & $\operatorname{Pn}^{147}$ & $\beta^{-} .23$ & & E .23 \\
\hline $2.6 \mathrm{~g}$ & $\mathrm{Na}^{22}$ & $\beta^{+} .54, \cdots$ & $\begin{array}{l}k \\
r \\
r\end{array}$ & E 2.84 \\
\hline $2.7 \mathrm{~g}$ & $\mathrm{Pu}^{236}$ & $\begin{array}{l}e^{-} 5.75, \cdots \\
\text { SF }\end{array}$ & $r \cdot \alpha_{4}$ & M 236.120 \\
\hline $2.7 \mathrm{~g}$ & $\mathrm{Sb}^{125}$ & $\mathrm{\beta}^{-} \cdot 30, \cdot .12-.62$ & $r .035, .42,(.11)-$ & E.76 \\
\hline $2.9 \mathrm{~J}$ & $P_{0}^{208}$ & a 5.11 & 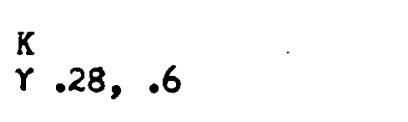 & M 208.046 \\
\hline $2.9 \mathrm{y}$ & $\mathrm{Fo}^{55}$ & - & $K$ & E .22 \\
\hline$>3.5 \mathrm{~g}$ & $a^{42}$ & $\beta^{-}$ & $\therefore$ & \\
\hline $3.7 \mathrm{~g}$ & $\mathrm{NB} 93$ & $e^{-}$ & I.T. . & \\
\hline $4.1 \mathrm{~g}$ & $\mathrm{~T}^{2 \mathrm{O}_{4}}$ & $\beta^{-} .76$ & $\mathbf{K}$ & $\begin{array}{l}\mathrm{E}^{-} .76 \\
\mathrm{E}^{+} \cdot 34\end{array}$ \\
\hline $5 \mathrm{y}$ & $\mathrm{Cd}^{113}$ & $\beta^{-} .58$ & & \\
\hline$\sim 5 \mathrm{y}$ & H. 172 & $0^{-} .2$ & $\begin{array}{l}K \\
\gamma \cdot 8, \cdot 3\end{array}$ & \\
\hline$\sim 58$ & $3 \mathrm{Rh}^{101}$ & & $\begin{array}{l}\text { I.T. }(3) \quad .127 \\
r .198\end{array}$ & . \\
\hline $5.2 \mathrm{~g}$ & $\mathrm{Co}^{60}$ & $\beta^{-} \cdot 31, \cdots$ & $r 1.33,1.17, \ldots$ & E 2.81 \\
\hline $6.7 \mathrm{~g}$ & $\mathrm{Ra}^{228}$ & $\beta^{-}<. \infty$ & : & $E<.05$ \\
\hline $8 \mathrm{~g}$ & $\mathrm{Ba}^{133}$ & $\theta^{-}$ & $\begin{array}{l}\mathrm{K} \\
r .082 \mathrm{D}, .36, .30, \\
.06, .07\end{array}$ & \\
\hline
\end{tabular}

- Total Disintegration Energy (Mov), or Isotopio Mass

Page 51. 


\begin{tabular}{|c|c|c|c|c|}
\hline Half-Lfe & Isotope & $\begin{array}{l}\text { Particles and } \\
\text { Energies (Mev) }\end{array}$ & $\begin{array}{l}\text { Camma Energies } \\
\text { (Mev) }\end{array}$ & $\begin{array}{l}\text { Total Energy } \\
\text { or Mass* } \\
\end{array}$ \\
\hline $8.0 \mathrm{y}$ & $\mathrm{Bi}^{207}$ & & $\begin{array}{l}\mathrm{K}, \mathrm{L} \\
\gamma .57,1.77, \ldots \\
(1.07, .57)\end{array}$ & E 2.4 \\
\hline $10 y$ & $\operatorname{cf}^{250}$ & $\underset{\mathrm{SF}}{a 6 . \propto,} 5.98$ & $r . \alpha_{4} 3$ & M 250.155 \\
\hline $10.4 \mathrm{~g} \ldots \ldots$ & $K r^{85} \cdots$ & $\beta^{-} .67, \cdots, \cdots$ & $\gamma .52 \cdots \cdots \cdots$ & E. .67 \\
\hline $12.26 \mathrm{y}$ & $\mathrm{H}^{3}$ & $\beta^{-} .018$ & no $Y$ & $E .018$ \\
\hline $13 \mathrm{y}$ & $\mathrm{Eu}^{152}$ & $\beta^{-} .70, \cdots$ & $\begin{array}{l}\mathrm{K} \\
\mathrm{r} .12 \mathrm{D}, .34,1.1, \cdots\end{array}$ & \\
\hline $13 \mathrm{y}$ & $\mathrm{Pu}^{241}$ & $\begin{array}{l}\beta^{-} .02 \\
\alpha 4.89,4.85\end{array}$ & $r .100, .145$ & E. .020 \\
\hline $16 y$ & $\mathrm{Eu}^{154}$ & $\beta-1.5, \cdots$ & $r_{i .12}^{.1230,1.42, .78,}$ & \\
\hline $18 y$ & $\mathrm{Cm}^{244}$ & $\underset{S F}{a} 5.80,5.76, \cdots$ & $\gamma . \alpha_{4} 3, \cdots$ & M 244.139 \\
\hline $20 y$ & $\mathrm{PO}^{210}$ & $\beta^{-} \cdot \infty 0$ & $r . \alpha_{4} 7, \cdots$ & E. .067 \\
\hline$>20 \mathrm{y}$ & $\mathrm{T}^{44}$ & & $\begin{array}{l}K \\
r .16\end{array}$ & \\
\hline $22 \mathrm{y}$ & $\mathrm{Ac}^{227}$ & $\begin{array}{l}\beta^{-} .046 \\
\alpha 4.94\end{array}$ & & M 227.099 \\
\hline $25 \mathrm{~g}$ & $\mathrm{Pm}^{145}$ & & $\begin{array}{l}K, L \\
r .068, .073\end{array}$ & \\
\hline $28 \mathrm{y}$ & $\mathrm{Sr}^{90}$ & $\beta^{-} .54,(2.2)$ & no $\gamma$ & E. .54 \\
\hline $30 \mathrm{y}$ & $\mathrm{Cs} 137$ & $\beta^{-} .52,1.18$ & $(\gamma .662)$ & E 1.18 \\
\hline$>30 \mathrm{y}$ & $\mathrm{Ho}^{166}$ & $\beta^{-} .2, \cdots$ & $\gamma .081 D-.84$ & E 2.4 \\
\hline $35 \mathrm{~g}$ & $\mathrm{Cm}^{243}$ & a $5.78,5.73,5.99, \ldots$ & $\begin{array}{l}.23, .28, .21 \\
.046-.33\end{array}$ & iv 243.137 \\
\hline$>35 y$ & $\mathrm{Gd}^{148}$ & a 3.2 & & \\
\hline $74 y$ & $u^{232}$ & $\begin{array}{l}a 5.32,5.26,5.13 \\
e^{-} \\
S F\end{array}$ & $\begin{array}{l}.058, .130, .27 \\
.33\end{array}$ & M 232.110 \\
\hline
\end{tabular}

* Total Disintegration Energy (Mev), or Isotopic Mass

Page 52. 


\begin{tabular}{|c|c|c|c|c|c|}
\hline Half-Iffe & Isotope & $\begin{array}{l}\text { Particles and } \\
\text { Energies (Mev) }\end{array}$ & $\begin{array}{l}\text { Garna Energies } \\
\frac{\text { (Mev) }}{}\end{array}$ & & $\begin{array}{l}\text { Total Ener: } \\
\text { or }\end{array}$ \\
\hline $80 y$ & $\operatorname{Sin}^{151}$ & $\beta^{-} .076$ & $r .000$ & & E.096 \\
\hline $80 \mathrm{y}$ & $\mathrm{Ni}^{63}$ & $\beta^{-} .063$ & no $r$ & & $E .063$ \\
\hline $90 \mathrm{y}$ & $\mathrm{Pu}^{238}$ & ${ }_{S F} 5.49,5.45, \cdots$ & $r .044, .100$ & .15 & M 238.124 \\
\hline$\sim 100 \mathrm{y}$ & $\mathrm{Am}^{242}$ & $\beta_{\alpha}^{-} .59, \cdots$ & $\begin{array}{l}K \\
\gamma . \alpha_{4}\end{array}$ & - & \\
\hline$\sim 100 \mathrm{y}$ & $\mathrm{Po}^{209}$ & a 4.88 & $\begin{array}{l}K \\
\gamma .90, .60, .20\end{array}$ & 29 & \\
\hline$\sim 140 \mathrm{y}$ & $\mathrm{Mn}^{53}$ & & $\begin{array}{l}k \\
\text { no } r\end{array}$ & & E. .60 \\
\hline $260 y$ & $A^{39}$ & $3^{-} .57$ & no $r$ & & E .57 \\
\hline$\sim 300 \mathrm{y}$ & $\mathrm{SI}^{32}$ & $\beta^{-} .1$ & no $r$ & & $E .1$ \\
\hline $470 \mathrm{y}$ & $\mathrm{Am}^{241}$ & $\begin{array}{l}\text { a } 5.48,5.38-5.54 \\
e^{-} \\
S F\end{array}$ & $r . \infty 0, .026-$ & .37 & M 241.132 \\
\hline $500 \mathrm{y}$ & $\mathrm{cf}^{249}$ & $a_{S F} 5.81,6.00, \cdots$ & $\gamma .40, .34, \cdots$ & & \\
\hline$\sim 700 y$ & $\mathrm{Cf}^{251}$ & a & & & \\
\hline $4000 \mathrm{y}$ & $\mathrm{Cm}^{246}$ & $\begin{array}{l}\text { a } 5.36 \\
\mathrm{SF}\end{array}$ & & & M 246.144 \\
\hline$\geq 5000 \mathrm{y}$ & $\mathrm{Np}^{236}$ & $\beta^{-}$ & & & M 236.121 \\
\hline $5600 \mathrm{y}$ & $c^{14}$ & $8^{-} .158$ & no $r$ & & E.158 \\
\hline $6600 \mathrm{~g}$ & $\mathrm{Pu}^{240}$ & $\propto 5.16,5.12, \ldots$ & $r \cdot 045$ & & M 240.129 \\
\hline 7000 у & $\mathrm{Bk}^{247}$ & a $5.50,5.67,5.30$ & $\gamma .085, .26$, & .42 & M 247.148 \\
\hline $7300 y$ & $\mathrm{Th}^{229}$ & a $4.85,4.94,5.02$ & & & M 229.103 \\
\hline$\sim 8000 \mathrm{~g}$ & $\mathrm{Am}^{243}$ & a $5.27,5.22,5.17-5.34$ & $r .075, \cdots$ & & M 243.137 \\
\hline $11,000 \mathrm{~g}$ & $\mathrm{Cm}^{245}$ & a $5.34, \cdots$ & $r .101, .17$ & & $M 245.142$ \\
\hline $20,000 \mathrm{y}$ & $\mathrm{Mb}^{94}$ & $\beta^{-} .5$ & $\gamma .87, .70,1$. & .57 & E 2.1 \\
\hline
\end{tabular}

* Total Disintegration Energy (Mev), or Isotopic Mass 


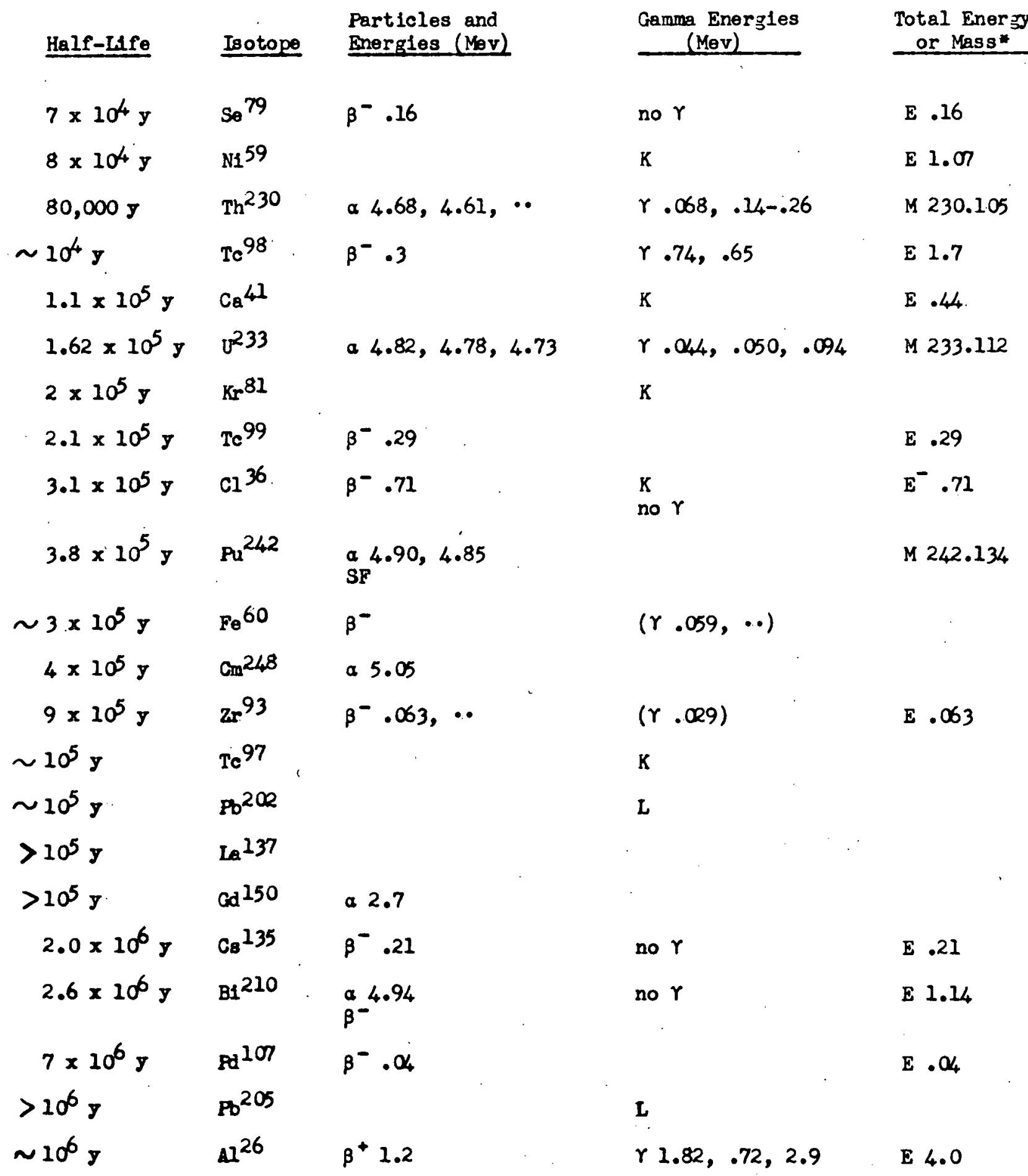

* Total Disintegration Bnergy (Mev), ar Isotopic Mass

Page 54. 


\begin{tabular}{|c|c|c|c|c|}
\hline Plf-Lffe & Isotope & $\begin{array}{l}\text { Particles and } \\
\text { Eneroles (Moy) }\end{array}$ & $\begin{array}{l}\text { Gamma Energles } \\
\frac{(\mathrm{Mev})}{}\end{array}$ & $\begin{array}{l}\text { Total Energy } \\
\text { or Mass: } \\
\end{array}$ \\
\hline $1.7 \times 10^{7} \mathrm{y}$ & $I^{129}$ & $\begin{array}{l}\beta^{-} .15 \\
e^{-}\end{array}$ & $r .038$ & E. .19 \\
\hline $2.39 \times 10^{7} \mathrm{y}$ & $0^{236}$ & $\begin{array}{l}\text { a } 4.50, \cdots \\
e^{-} \\
S F\end{array}$ & $r .05$ & M 236.120 \\
\hline$\sim 5 \times 10^{7} \mathrm{y}$ & $S_{m} 246$ & $\alpha 2.5$ & & \\
\hline $8 \times 10^{7} \mathrm{y}$ & $\mathrm{Pu}^{244}$ & $\begin{array}{l}a \\
S F\end{array}$ & & \\
\hline $1.3 \times 10^{9} \mathrm{y}$ & $K^{40}$ & $\beta^{-}$1.33, & $\begin{array}{l}K \\
r 1.46\end{array}$ & $E^{-1.33}$ \\
\hline
\end{tabular}

* Total Disintegration Energy (Mav), or Isotopic Mass

Page 55. 
Table of thermal neutron absorption cross-sections. Values are in barns.

\begin{tabular}{|c|c|c|c|c|c|}
\hline Isotope & Half-Lffe & Cross-Section & Isotope & Helf-Lfe & Cross-Section \\
\hline $\mathrm{Be}^{7}$ & $53 \mathrm{~d}$ & $\mathrm{np} \sim 10^{4}$ & $\mathrm{Cs}^{135}$ & $2.0 \times 10^{6} \mathrm{y}$ & $\sim 15$ \\
\hline$c^{14}$ & $5600 \mathrm{y}$ & $<10^{-6}$ & $\mathrm{Cs}^{137}$ & $30 \mathrm{~g}$ & $<2$ \\
\hline C136 & $3.1 \times 10^{5} \mathrm{y}$ & $\sim 90$ & $\mathrm{Ba} 139$ & $1.42 \mathrm{~d}$ & 4 \\
\hline$A^{41}$ & $1.82 \mathrm{~h}$ & $>.06$ & $\mathrm{La}^{140}$ & $1.67 \mathrm{~d}$ & 3 \\
\hline$k^{40}$ & $1.3 \times 10^{9} \mathrm{~J}$ & 70 & $\mathrm{Ce}^{143}$ & $1.37 \mathrm{~d}$ & 6 \\
\hline$S c^{46}$ & $85 d$ & .25 & $\operatorname{Pr}^{142}$ & $19.1 \mathrm{~h}$ & 20 \\
\hline $\mathrm{Co}^{60}$ & $5.2 \mathrm{~J}$ & 6 & $\operatorname{Pu} 147$ & $2.6 y$ & $\left(\sim 60^{*}+?\right)$ \\
\hline $\mathrm{Co}^{60}$ & $10.5 \mathrm{~m}$ & $\sim 100$ & $\mathrm{Sm}^{151}$ & $80 \mathrm{y}$ & 12,000 \\
\hline $\mathrm{Cu}^{66}$ & $5.1 \mathrm{~m}$ & 140 & Eu 152 & $13 y$ & 5000 \\
\hline$k r^{85}$ & $10.4 \mathrm{~g}$ & $<15$ & $\mathrm{Bu}^{154}$ & $16 y$ & 1400 \\
\hline$K r^{87}$ & $1.30 \mathrm{~h}$ & $<600$ & $\mathrm{Eu}^{155}$ & $1.7 \mathrm{y}$ & 13,000 \\
\hline $\mathrm{Rb} \mathrm{b}^{88}$ & $18 \mathrm{~m}$ & $<200$ & $\mathrm{~Tb}^{160}$ & $72 \mathrm{~d}$ & $\sim 600$ \\
\hline $\mathrm{Sr}{ }^{89}$ & $54 d$ & $<130$ & $D_{y}^{165}$ & $2.32 \mathrm{~h}$ & 4700 \\
\hline $\mathrm{Sr} 90$ & $28 \mathrm{y}$ & 1 & $\operatorname{Tn}^{170}$ & $129 \mathrm{~d}$ & $\sim 2000$ \\
\hline$Y^{90}$ & $2.67 \mathrm{~d}$ & 6 & $\mathrm{Ta}^{182}$ & $112 \mathrm{~d}$ & $\sim 20,000$ \\
\hline $2 x^{93}$ & $9 \times 10^{5} \mathrm{y}$ & $<5$ & $w^{187}$ & $1 d$ & $\sim 80$ \\
\hline $\mathrm{Nb}^{9 / 4}$ & $20,000 y$ & $\sim 15$ & $\operatorname{Re}^{188}$ & $17 \mathrm{~h}$ & $<3$ \\
\hline $\mathrm{Tc}^{99}$ & $2.1 \times 10^{5} \mathrm{~g}$ & 20 & $\infty^{193}$ & $1.29 \mathrm{~d}$ & 200 \\
\hline$I^{129}$ & $1.7 \times 10^{7} \mathrm{~g}$ & 30 & $\mathrm{Au}^{198}$ & $2.70 d$ & 26,000 \\
\hline$I^{131}$ & $8.05 d$ & $\sim 600$ & $\mathrm{Au}^{199}$ & $3.15 \mathrm{~d}$ & $\sim 30$ \\
\hline$x_{\theta} 135$ & $9.2 \mathrm{~h}$ & $\left(3.2 \times 10^{6}\right)$ & $\operatorname{Em}^{222}$ & $3.825 d$ & .7 \\
\hline
\end{tabular}

* To metastable state. 


\begin{tabular}{|c|c|c|c|c|c|}
\hline Isotope & Half-Lfe & Cross-Soction & Isotope & Half-Llfe & Cross-Section \\
\hline $\mathrm{Ra}^{223}$ & $11.6 \mathrm{~d}$ & 125 & $\mathrm{Pu}^{241}$ & $13 \mathrm{y}$ & 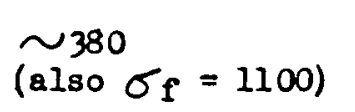 \\
\hline $\mathrm{Re}_{2} 224$ & $3.64 d$ & 12 & $\mathrm{Pu}^{242}$ & $3.8 \times 10^{5} \mathrm{y}$ & 23 \\
\hline $\mathrm{Re}^{228}$ & $6.7 \mathrm{~g}$ & $\sim 36$ & $\mathrm{Pu}^{243}$ & $5.0 \mathrm{~h}$ & $\sim 100$ \\
\hline $\mathrm{Ac}^{227}$ & $22 \mathrm{y}$ & $\sim 520$ & $\mathrm{Pu}^{244}$ & $8 \times 10^{7} \mathrm{y}$ & $\sim 1.4$ \\
\hline$T h^{227}$ & $18.2 \mathrm{~d}$ & $(\mathrm{f}) \sim 1500$ & $\mathrm{Pu}^{245}$ & $11 \mathrm{~h}$ & $\sim 260$ \\
\hline $\begin{array}{l}T^{228} \\
T h^{229}\end{array}$ & $\begin{array}{l}1.90 \mathrm{y} \\
7300 \mathrm{y}\end{array}$ & $\begin{array}{l}120 \\
(f) \sim 45\end{array}$ & $\mathrm{Am}^{241}$ & $470 y$ & $\begin{array}{l}(700 *+<50) \\
\left(\text { also } \sigma_{f}=3.2\right)\end{array}$ \\
\hline $\mathrm{Th}^{230}$ & $80,000 \mathrm{z}$ & 35 & $\mathrm{Am}^{242}$ & $\sim 100 \mathrm{~g}$ & 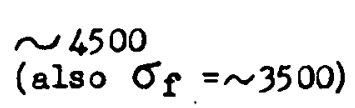 \\
\hline $\begin{array}{l}T h^{233} \\
T h^{234}\end{array}$ & $\begin{array}{l}23.3 \mathrm{~m} \\
24.10 \mathrm{~d}\end{array}$ & $\begin{array}{l}1400 \\
1.8\end{array}$ & $\operatorname{Am}^{2 / 42}$ & $16.0 \mathrm{~h}$ & $(f) \sim 2500$ \\
\hline $\mathrm{Pa}^{230}$ & $17 \mathrm{~d}$ & (f) 1500 & $\operatorname{Am}^{243}$ & $\sim 8000 \mathrm{y}$ & 82 \\
\hline $\mathrm{Pa}^{232}$ & $1.31 \mathrm{~d}$ & 700 (also $\left.\sigma_{f}=700\right)$ & $\mathrm{Cm}^{2}$ & $163 d$ & $\sim 20$ \\
\hline $\mathrm{Pa}^{233}$ & $27.4 d$ & $\left(40^{4}+26\right)$ & $a^{<4>}$ & $35 \mathrm{~g}$ & $\sim 300$ \\
\hline$v^{230}$ & $21 \mathrm{~d}$ & $(f) \sim 25$ & 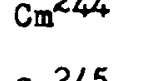 & $18 \mathrm{~g}$ & $\sim 15$ \\
\hline $0^{231}$ & $4.3 d$ & $(f) \sim 400$ & $\mathrm{Cm}^{245}$ & $11,000 \mathrm{y}$ & 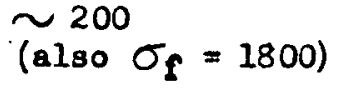 \\
\hline$v^{232}$ & $74 \mathrm{~J}$ & $\sim 300$ (also $\left.\sigma_{\mathrm{f}}=80\right)$ & $\mathrm{Cm}^{246}$ & $4000 \mathrm{y}$ & $\sim 15$ \\
\hline$u^{233}$ & $1.62 \times 10^{5} \mathrm{y}$ & 60 (also $\left.\sigma_{f}=520\right)$ & $\mathrm{Cm}^{247}$ & $\gg 18$ & 180 \\
\hline$U^{236}$ & $2.39 \times 10^{7} \mathrm{y}$ & 8 & $\mathrm{Bk}^{249}$ & $290 d$ & $\sim 500$ \\
\hline$u^{239}$ & $23.5 \mathrm{~m}$ & $22\left(\right.$ also $\left.\sigma_{f}=\sim 12\right)$ & $\mathrm{Cf}^{249}$ & $500 \mathrm{y}$. & $\left.\sim_{(0130}^{270} \sigma_{f}=\sim 600\right)$ \\
\hline $\mathrm{Np}^{23 / 4}$ & & $(f) \sim 900$ & $\mathrm{Cr}^{250}$ & $10 \mathrm{y}$ & $\sim 1500$ \\
\hline $\begin{array}{l}\mathrm{Np}<30 \\
\mathrm{~Np} 238\end{array}$ & $\begin{array}{l}\geq 5000 \\
2.10 \mathrm{~d}\end{array}$ & $\begin{array}{l}\text { (f) } 2800 \\
\text { (f) } 1600\end{array}$ & $\mathrm{Cr}^{251}$ & $\sim 700 \mathrm{y}$ & $\sim 3000$ \\
\hline $\mathrm{Np}^{239}$ & $2.33 d$ & 80 & $\begin{array}{l}\mathrm{Cf}^{252} \\
\mathrm{Cf}^{254}\end{array}$ & $2.2 \mathrm{y}$ & $\begin{array}{l}\sim 30 \\
<2\end{array}$ \\
\hline $\begin{array}{l}\mathrm{Pu}^{238} \\
\mathrm{Pu}^{240}\end{array}$ & $\begin{array}{l}90 \mathrm{y} \\
6600 \mathrm{y}\end{array}$ & $\begin{array}{l}450\left(\mathrm{also} \sigma_{\mathrm{f}}=18\right) \\
\sim 510\end{array}$ & $E^{253}$ & $20 \mathrm{~d}$ & $\sim 200$ \\
\hline
\end{tabular}

- To motastable state. 
Isotope Half-Life Cross-Section

$\begin{array}{lll}E^{254} & 1.54 \mathrm{~d} & \leq 15 \\ \mathrm{E}^{255} & \sim 30 \mathrm{~d} & \sim 40 \\ \mathrm{Fm}^{255} & 20 \mathrm{~h} & <100\end{array}$

Append ix Page 3. 